\title{
Middle and Late Jurassic radiolarians from the Neotethys suture in the Eastern Alps
}

\author{
Luis O’Dogherty, ${ }^{1}$ Špela Goričan, ${ }^{2}$ and Hans-Jürgen Gawlick ${ }^{3}$ \\ ${ }^{1}$ Departamento de Ciencias de la Tierra, Universidad de Cádiz, CASEM, E-11510 Puerto Real, Spain 〈luis.odogherty@uca.es〉 \\ ${ }^{2}$ Paleontološki inštitut Ivana Rakovca, ZRC SAZU, Novi trg 2, SI-1000 Ljubljana, Slovenia 〈spela@ zrc-sazu.si〉 \\ ${ }^{3}$ University of Leoben, Department of Applied Geosciences and Geophysics, Petroleum Geology, Peter-Tunner-Strasse 5, 8700 Leoben, Austria \\ 〈Hans-Juergen.Gawlick@unileoben.ac.at〉
}

\begin{abstract}
Cherty limestones, marls and radiolarites are widespread in the Hallstatt Mélange of the Northern Calcareous Alps. The mélange was formed during the Neotethyan orogeny in a series of deep-water basins that progressively developed in front of the advancing nappe front. The low thermal overprint of these rocks favors the good preservation of radiolarians (polycystines) that have been used for dating and reconstructing the Jurassic tectonostratigraphy of the area. This paper describes rich Middle-Late Jurassic radiolarian faunas from four localities in the Hallstatt Mélange near Bad Mitterndorf in Austria. Two different successions, both spanning from the Bathonian to the Oxfordian, are dated. In the first succession, the radiolarites are intercalated between or occur as matrix in mass-flow deposits originating from the accretionary wedge. The second succession is nearly $100 \mathrm{~m}$ thick but is devoid of mass-flow deposits and documents a continuous radiolarite deposition in greater distance from the nappe stack. Both successions are ascribed to the Sandlingalm Basin, which evolved on a relatively distal continental margin during early stages of the orogeny.

The highly diverse and well-preserved radiolarian assemblages have been used for a detailed taxonomic study. Two new families are described: Minocapsidae n. fam. and Xitomitridae n. fam.; six new genera are described Doliocapsa n. gen., Crococapsa n. gen., Parvimitrella n. gen., Xitomitra n. gen., Campanomitra n. gen., and Mizukidella n. gen. In addition, one new replacement name, Takemuraella (pro Triversus), is introduced, the diagnoses of 6 genera are emended, and two new species are described: Hemicryptocapsa nonaginta n. sp. and Mizukidella mokaensis n. sp.
\end{abstract}

\section{Introduction}

This paper describes rich Middle and Late Jurassic radiolarian faunas from four localities in the Hallstatt Mélange near Bad Mitterndorf in Austria. Radiolarian assemblages are well preserved, suitable for taxonomic studies, and precise biostratigraphy.

Middle and Late Jurassic low-latitude radiolarians have been relatively well studied in terms of species-level systematics and biochronology (Baumgartner et al., 1995a, b); however, the systematics of genera and families has not been sufficiently elaborated yet. O'Dogherty et al. (2009) presented an illustrated catalogue of type species of Jurassic and Cretaceous genera described so far. All genera were revised, and many were considered as invalid (synonyms, homonyms, nomina dubia). Another group of radiolarians that needs taxonomic revision are those Mesozoic species that still bear inappropriate names of Recent genera. The structure of the catalogue, consisting exclusively of illustrations and synonymy, did not allow us to discuss the distinguishing characteristics of the valid genera or to describe new taxa. The primary aim of this paper is to move towards a more natural taxonomy of Jurassic radiolarians. Six new genera are described, a replacement name for a homonym is proposed, the diagnoses of six valid genera are emended, and remarks for several other genera are provided to clarify their definition. For all new and revised genera, a list of included species is presented. Two new families are erected and two new species are described.

In addition to radiolarian taxonomy, this paper contributes to the biostratigraphic data of the Hallstatt Mélange in the Northern Calcareous Alps. Extensive radiolarian dating over the last 15 years has provided a wealth of age constraints in deep-water sediments of the Tirolic units and the Hallstatt Mélange (Suzuki and Gawlick, 2003, 2009; Missoni and Gawlick, 2011a, b and references therein). These data had important implications for the reconstruction of the Jurassic tectonostratigraphy and distinguished several trench-like basins that formed progressively during the propagation of thrusting (see Missoni and Gawlick, 2011a, b; Gawlick et al., 2012 for the latest reviews). However, the structure of this area, especially that of the Hallstatt Mélange, is extremely complex and the proposed tectonostratigraphic model can still be refined with additional biostratigraphic data.

\section{Geological overview}

The study area is located in the central Northern Calcareous Alps around $100 \mathrm{~km}$ southeast of Salzburg (Fig. 1.1). The Northern Calcareous Alps represent a far travelled nappe system 
Juvavic units (provenance of the Meliata and Hallstatt Mélange

Tirolic units and equivalents with Meliata, Hallstatt, Tauglboden and Rofan Mélange

Bavaric units and equivalents

Lower Austroalpine and Central Alpine Mesozoic units and equivalents

E: :- Palaeozoic units, weakly metamorphosed

Crystalline basement

Penninic and Helvetic zones

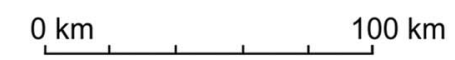

major disintegration lines during

Miocene lateral extrusion
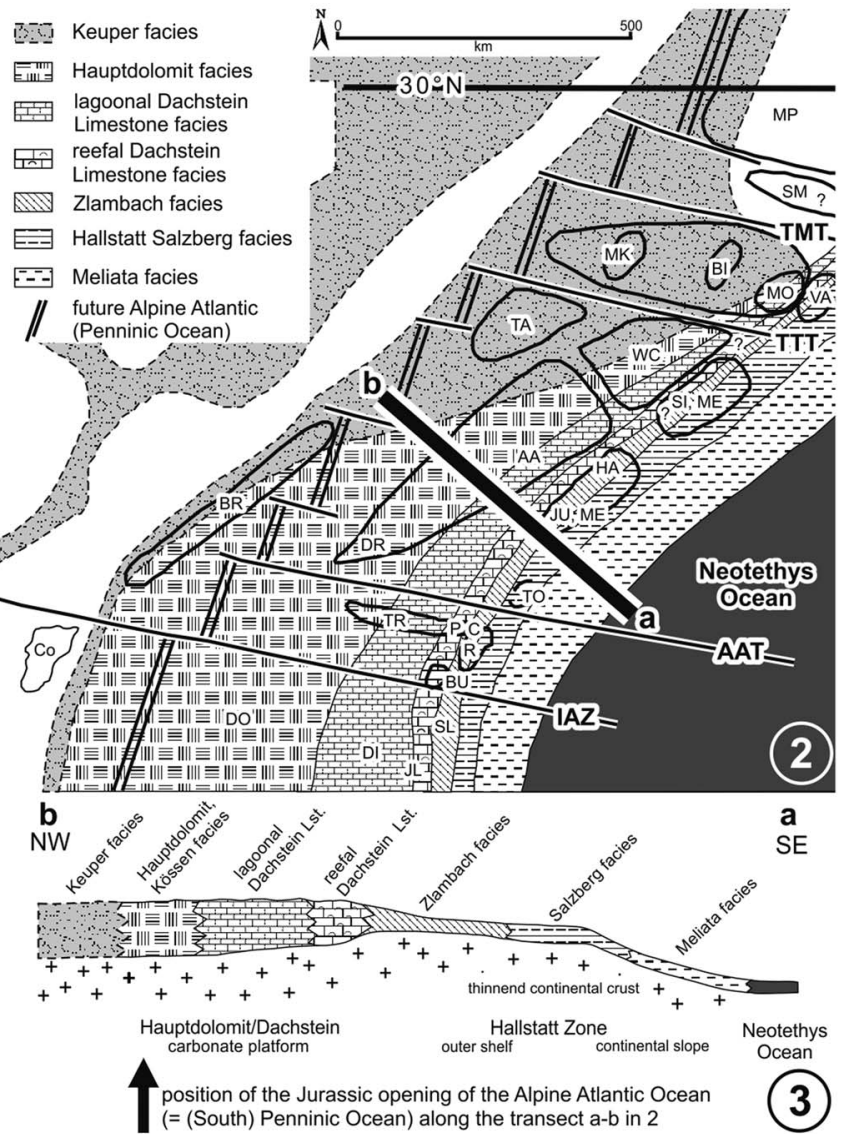

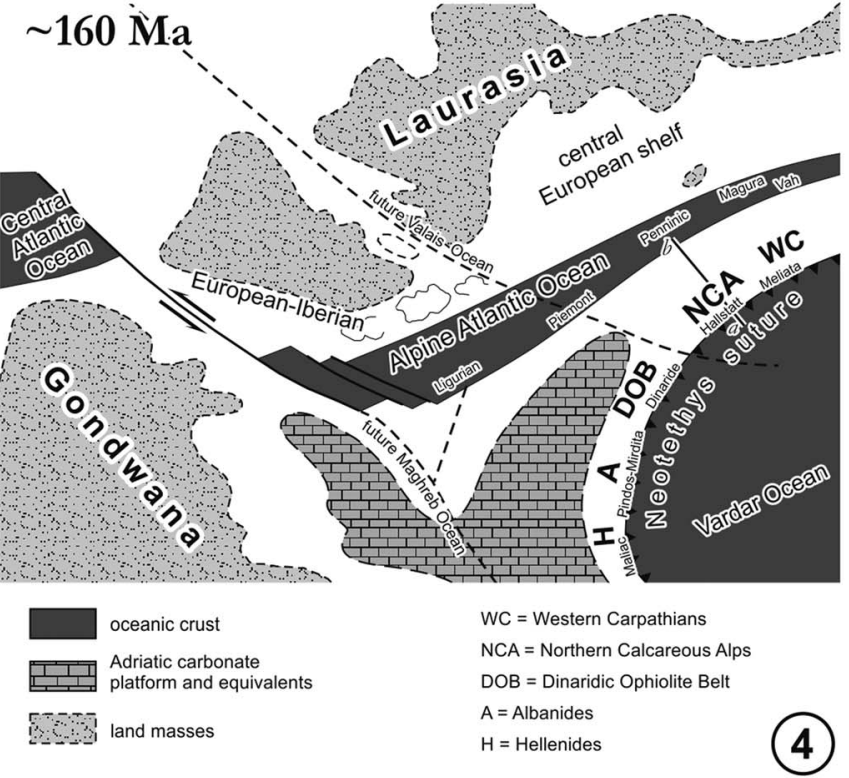

b

a NW

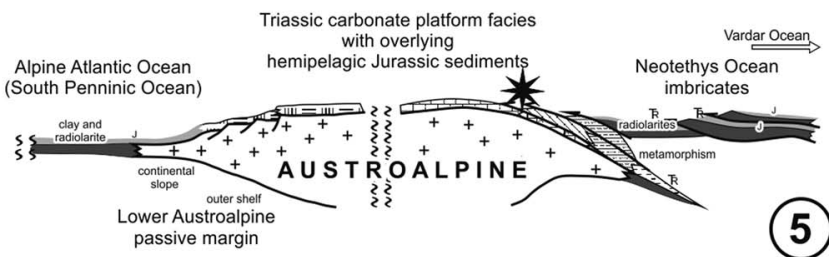


in the highest structural position of the Eastern Alps (Tollmann, 1985) and belong palaeogeographically to the Austroalpine domain (Fig. 1.1).

In Middle-Late Triassic times the Austroalpine domain as part of the central and southeastern European shelf show a typical carbonate passive continental margin facies distribution (Fig. 1.2-1.3), whereas in Jurassic times the Austroalpine realm was situated between the Penninic Ocean to the northwest and the Neotethys Ocean in the southeast (Fig. 1.4-1.5).

Contemporaneous with progressive Jurassic extension and opening of the Alpine Atlantic Ocean (and the Penninic realm as part of it; see Missoni and Gawlick, 2011a for details) as an eastward continuation of the Central Atlantic Ocean, closure of parts of the Neotethys Ocean with formation of the Neotethys ophiolite imbricates started in the late Early Jurassic and prevailed until the early Late Jurassic (Karamata, 2006). Obduction of the Neotethys ophiolite imbricates started in the Middle Jurassic and the Austroalpine and its northern and southern equivalents attained a lower plate position (Frisch and Gawlick, 2003, Gawlick et al., 2008, Schmid et al., 2008). Westward to northwestward propagating Middle to early Late Jurassic compression led to the imbrication of the Austroalpine domain and its equivalents along the Neotethys suture and resulted in the Neotethyan orogeny (Missoni and Gawlick, 2011a). A characteristic feature of this orogenesis is the formation of deep-water radiolaritic basins in front of the westward propagating nappe stack. Sediment supply in these basins derived from the nappe fronts.

Gawlick et al. (1999) interpreted this sedimentation pattern as a reflection of nappe movements in the Northern Calcareous Alps in the late Middle to early Late Jurassic and related it to the Kimmeric orogeny according to earlier authors (see 'Jurassic gravitational tectonics' in Plöchinger, 1974, 1976; Tollmann, 1981, 1985, 1987; Mandl, 1982). This orogenic event (Lein, $1985,1987 \mathrm{a}, \mathrm{b})$ was related to the closure of the western half of the Neotethys Ocean, today named the Neotethyan orogeny (Missoni and Gawlick, 2011a).

In the southern and therefore highest nappe group (Tirolic units and equivalents, Fig. 1.2-1.3) of the Northern Calcareous Alps the remains of this orogenic event are well preserved with a series of deep-water radiolaritic basins which were formed in sequence in front of the advancing nappe front (e.g., Gawlick et al., 1999, Missoni and Gawlick, 2011b). These southernmost radiolarite basins contain the Hallstatt Mélange as an erosional product of the Juvavic nappes, which are mainly eroded today. Subsequently the trench-like basins became overthrusted and incorporated into the accretionary prism (Missoni and Gawlick, 2011b). This Hallstatt Mélange was therefore formed in the late Early to early Late Jurassic interval as a result of successive shortening of the Triassic to Jurassic distal shelf area (Hallstatt Zone).

\section{Jurassic evolution of the southern Northern Calcareous Alps}

In the early Early Jurassic, sedimentation was generally controlled by the topography of the Late Triassic Hauptdolomit/ Dachstein carbonate platform (Böhm, 2003, Gawlick and Frisch, 2003; Figs. 1, 2). On top of the Rhaetian shallow-water carbonates, red condensed limestones of the Adnet Group (?late Hettangian/Sinemurian to Toarcian: Böhm, 1992, 2003) were deposited, mostly separated by a gap of sedimentation (mainly early Hettangian, partly also late Hettangian; Fig. 2). On top of the Rhaetian Kössen Formation (e.g., Eiberg Basin, Restental Basin, Fig. 2) cherty and marly bedded limestones (Kendlbach Formation; Scheibelberg Formation: Böhm, 1992, 2003; Krainer and Mostler, 1997; Ebli, 1997) were deposited, while in marginal areas of the basins crinoidal or sponge-spicule rich limestones of the Enzesfeld Formation were laid down (Böhm, 1992). In the late Pliensbachian to early Toarcian a horst-and-graben morphology developed (Bernoulli and Jenkyns, 1974, Krainer et al., 1994) and triggered breccia formation along submarine slopes and escarpments (Böhm et al., 1995). The Toarcian and most of the Middle Jurassic are characterized by starved sedimentation, ferro-manganese crusts, or a hiatus on the horsts, whereas the grabens were filled with deep-water carbonates and breccias, which latter formed near fault scarps. Neptunian dykes are found on the horsts. In the newly formed basinal areas gray bedded limestones of the younger Allgäu Formation were deposited, while condensed red limestones of the Klaus Formation formed on the top of the topographic highs (Krystyn, 1971, 1972; Fig. 2).

This sedimentation pattern changed dramatically in the late Middle Jurassic (Gawlick and Frisch, 2003). Sedimentation resumed with the deposition of radiolarian cherts and radiolaria-rich

Figure 1. Tectonic and paleogeographic maps. (1) Tectonic sketch map of the Eastern Alps and study area (after Tollmann, 1987; Frisch and Gawlick, 2003); GPU Graz Palaeozoic Unit; GU Gurktal Unit; GWZ Greywacke Zone; RFZ Rhenodanubian Flysch Zone. Star indicates study area (Fig. 3). (2) Late Triassic paleogeographic position and facies zones of the Austroalpine domain as part of the northwestern Neotethys passive margin; IAZ = Iberia-Adria Zone transform fault, AAT = future Austroalpine-Adria transform fault, TTT = future Tisza-Tatra transform fault, TMT = future Tisza-Moesia transform fault, $\mathrm{AA}=$ Austroalpine, $\mathrm{BI}=$ Bihor, $\mathrm{BR}=$ Briançonnais, $\mathrm{BU}=\mathrm{Bükk}, \mathrm{C}=\mathrm{Csovar}, \mathrm{Co}=\mathrm{Corsica}, \mathrm{DI}=\mathrm{Dinarides}, \mathrm{DO}=\mathrm{Dolomites}, \mathrm{DR}=\mathrm{Drau} \mathrm{Range}$, $\mathrm{HA}=$ Hallstatt Zone, JU = Juvavicum, JL = Julian Alps, ME = Meliaticum, MK = Mecsek, MO = Moma unit, MP = Moesian platform, P = Pilis-Buda, $\mathrm{R}=$ Rudabanyaicum, SI = Silicicum, SL = Slovenian trough, SM = Serbo-Macedonian unit, TA = Tatricum, TO = Tornaicum, TR = Transdanubian Range, VA $=$ Vascau unit, WC = central West Carpathians (modified after Haas et al., 1995; Gawlick et al., 1999, 2008). (3) Schematic cross section (for position, see line a-b in 2) showing the typical passive continental margin facies distribution across the Austroalpine domain in Late Triassic time (after Gawlick and Frisch, 2003). (4) Palaeogeographic position of the Northern Calcareous Alps as part of the Austroalpine domain in Late Jurassic time (after Frisch, 1979; Gawlick et al., 2008). In this reconstruction the Northern Calcareous Alps are part of the Jurassic Neotethyan Belt (orogen) striking from the Carpathians to the Hellenides. The Neotethys suture is equivalent to the obducted West-Vardar ophiolite complex (e.g., Dinaric Ophiolite Belt) in the sense of Schmid et al. (2008) = far-travelled ophiolite nappes of the western Neotethys Ocean in the sense of Gawlick et al. (2008) (see Robertson, 2012 for discussion). The eastern part of the Neotethys Ocean remained open $=$ Vardar Ocean (Missoni and Gawlick, 2011a). Toarcian to Early Cretaceous Adria-Apulia carbonate platform and equivalents according to Golonka (2002), Vlahović et al. (2005), and Bernoulli and Jenkyns (2009). (5) Schematic cross section reconstructed for Middle to Late Jurassic times showing the passive continental margin of the Lower Austroalpine domain facing the Penninic Ocean to the northwest (e.g., Tollmann, 1985; Faupl and Wagreich, 2000) and the lower plate position and imbrication of the Austroalpine domain in relation to the obducted Neotethys oceanic crust (after Gawlick et al., 2008; compare with Frisch, 1979). Star indicates position of study area (compare Figure 3). 


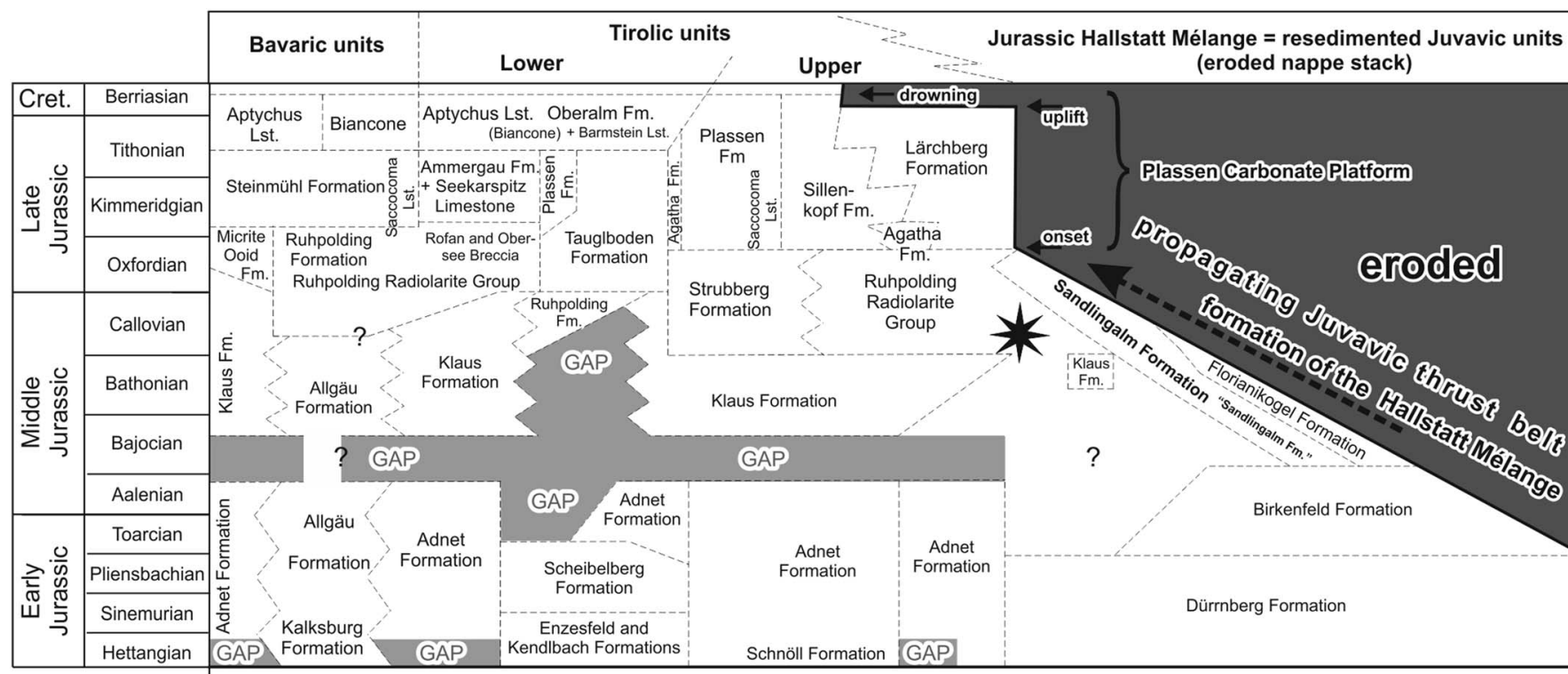

Palaeotopography of the Austroalpine around the Triassic/Jurassic boundary

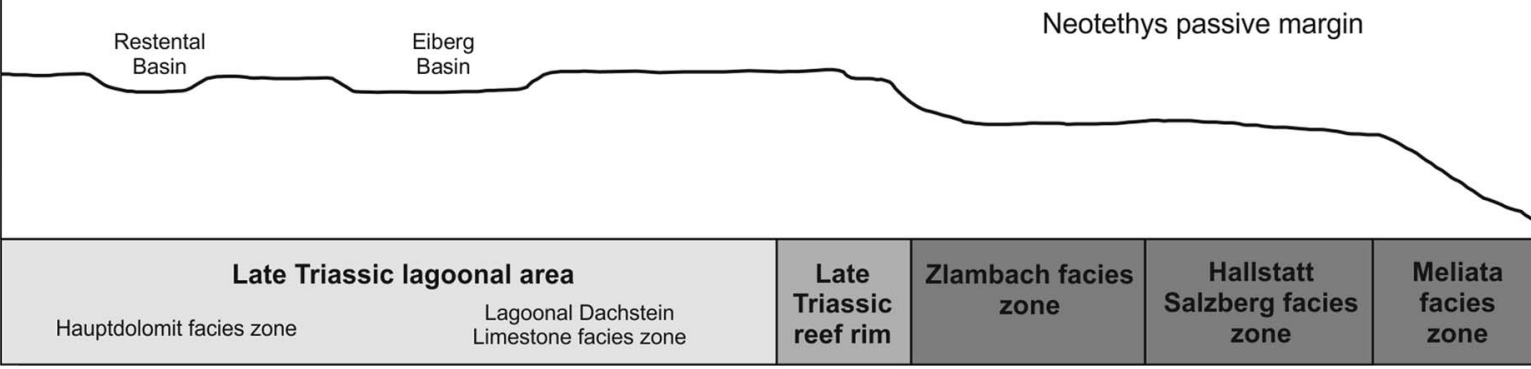

Middle Jurassic to Oxfordian: Formation of the Tirolic nappes

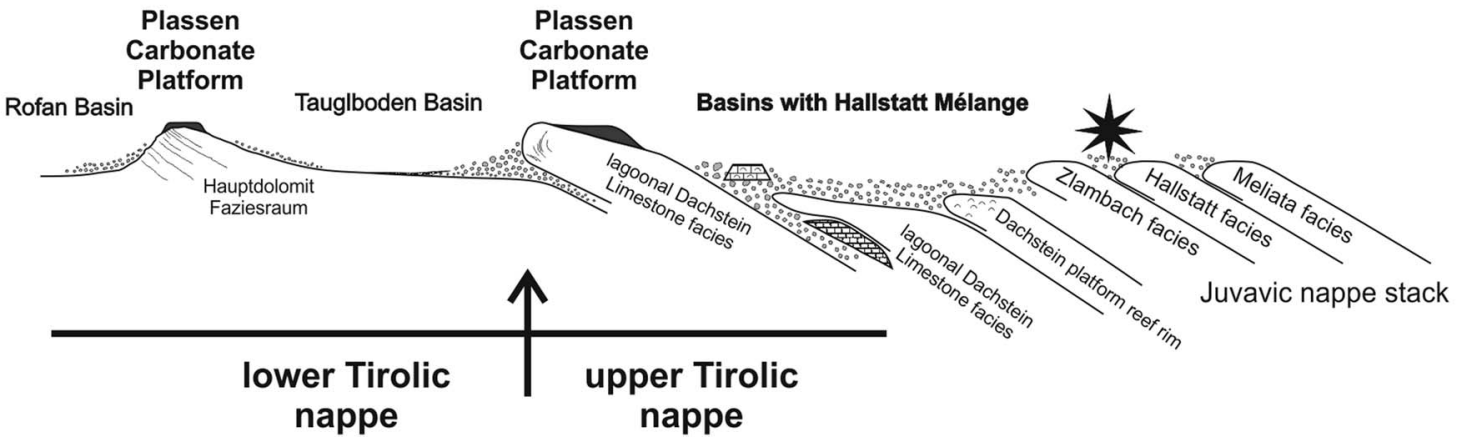

Figure 2. Stratigraphic table with lithostratigraphic names and main tectonic events of the Jurassic in the Austroalpine realm with their variations depending on the palaeogeographic position (after Gawlick et al., 2009); star indicates investigated sequence. Note that this sequence is thrust further northward to its present position during younger shortening events. Bavaric units, Tirolic units, and the Hallstatt Mélange belong to the Northern Calcareous Alps.

marls, shales, and limestones of the Ruhpolding Radiolarite Group (Diersche, 1980; Gawlick and Frisch, 2003; Fig. 2).

In the Bajocian the sedimentary evolution in the southern (palaeogeographically southeastern, Fig. 1.4-1.5) part of the Tirolic realm as well as in the Hallstatt realm (Fig. 2) differed from that in the northern part (palaeogeographically in the northwestern, see Fig. 1.4-1.5). Deep-water trench-like basins formed in front of advancing nappes. The first basin group in the southern parts of the Northern Calcareous Alps received mass-flow deposits and large slides up to nappe size, which derived from the Hallstatt Zone (= Hallstatt Mélange;
Gawlick and Frisch, 2003). The thickness of the basin fills may reach 2000 meters (Gawlick, 1996, 1997, Gawlick et al., 2007b). The nappe stack carrying the Hallstatt Mélange is defined as the upper Tirolic nappe (group) (Frisch and Gawlick, 2003). The second basin group (Fig. 2), the Tauglboden and the Rofan trench-like basins in the north, was subjected to high subsidence and sedimentation rates in the Oxfordian to earliest Kimmeridgian (Schlager and Schlager, 1973, Gawlick and Frisch, 2003). The nappe stack carrying the Tauglboden Mélange is defined as the lower Tirolic nappe (Frisch and Gawlick, 2003). These two basin groups are different: the huge 
mass flows of the Hallstatt Mélange trench-like basins formed earlier from material derived from the outer shelf facing the Neotethys Ocean (Hallstatt Zone, Fig. 1.2-1.5), whereas the Tauglboden Mélange trench-like basin formed later from material derived of the lagoonal part of the Hauptdolomit/ Dachstein carbonate platform (Fig. 1.4, 1.5). However, both basins formed syntectonically and suggest a substantial relief between the basin axis and the source area. A third type of radiolarite basin, the Sillenkopf Basin (Missoni et al., 2001), remained in the southern part of the Northern Calcareous Alps as a starved basin in the Kimmeridgian (Fig. 2). This basin contains the earliest ophiolitic detritus from the accreted and obducted Neotethys Ocean floor (Missoni, 2003).

In the Tirolic units of the Northern Calcareous Alps the establishment of the shallow-water Plassen Carbonate Platform started at the frontal parts of the rising and advancing nappes (Gawlick et al., 2002, 2005). From this position, the progradation of several independent platforms took place towards the adjacent radiolarite basins (Gawlick and Frisch, 2003; Gawlick and Schlagintweit, 2006; Gawlick et al., 2005, 2007a, 2012). This resulted in a complex basin-and-rise topography with different types of sediments in shallow-water and deep-water areas (Gawlick and Schlagintweit, 2006). In the Kimmeridgian a huge carbonate platform was formed in the upper Tirolic unit, whereas in the lower Tirolic unit shallowwater carbonates were restricted to its northern part (Gawlick et al., 2007a). The whole Plassen Carbonate Platform cycle lasted from the Kimmeridgian until the late early Berriasian platform drowning (Gawlick and Schlagintweit, 2006).

\section{Description of the studied localities}

All studied localities belong to the Hallstatt Mélange around the village of Obersdorf north of Bad Mitterndorf (Fig. 3). For a more detailed description of the geology of the area, the Late Triassic to Late Jurassic sedimentary succession, and radiolarian and conodont dating, see O’Dogherty and Gawlick (2008).

Kumitzberg.- The Late Triassic Hallstatt Limestone block of Mt. Kumitzberg is surrounded by a grassland area without outcropping sedimentary rocks (Fig. 3). Only small pieces of darkgray to black radiolarites can be found at the western base of Mt. Kumitzberg. During the reconstruction of a small bus station in the excavation hole the contact between the Hallstatt Limestone block and the underlying dark-gray to black radiolarite was visible. The contact between the Hallstatt Limestone block and the radiolarite is erosive. This clearly indicates that the massive limestone block cut deep into the radiolarite succession. Therefore the age of the radiolarites is slightly older than the time of its emplacement. The unlaminated and massive dark-gray radiolarite beds are intercalated by thin layers of cherty shales. Only one sample (EW-158) was collected from this locality.

Steinwand north.-A slightly folded, relative thick radiolarite succession is preserved in a valley between the Steinwand and the Mischenirwiese (Fig. 3), on the southeastern slope of Mount Kampl. This succession occurs on top of the Late Triassic (Rhaetian) lagoonal Dachstein Limestone of the Steinwand (Fig. 4). The overlying Early Jurassic interval is covered by

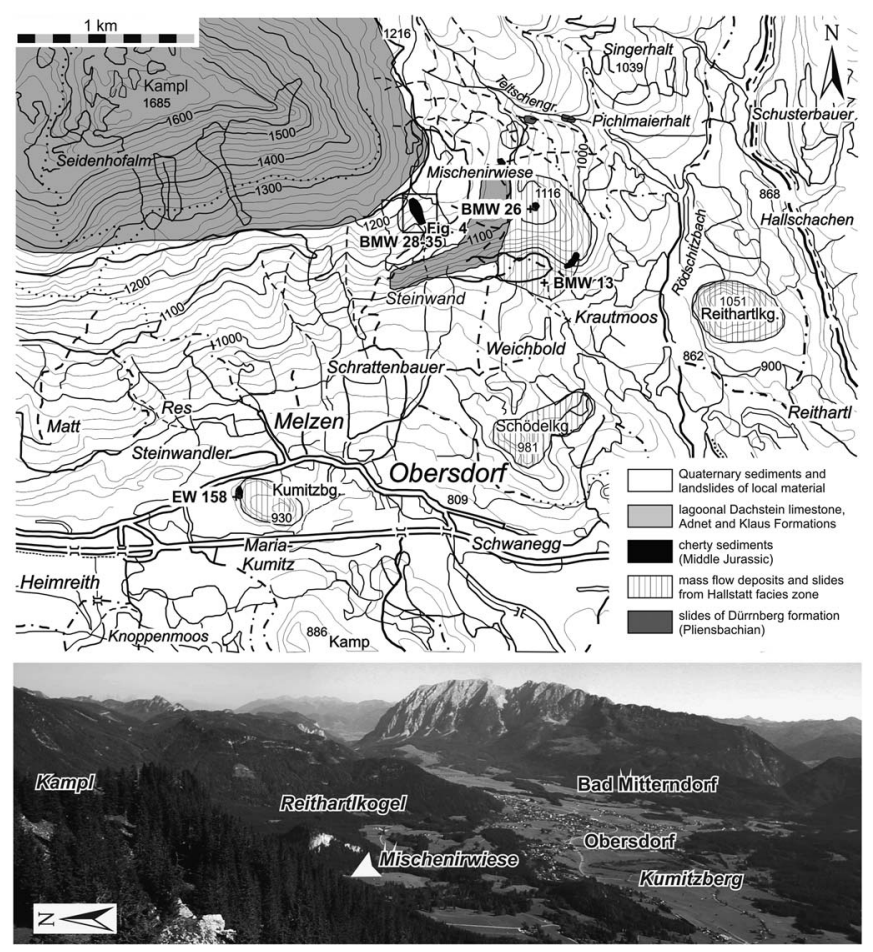

Figure 3. Topography and simplified geology of the study area, showing sample locations (after O'Dogherty and Gawlick, 2008). The plus signs indicate positions of the investigated samples below the Kumitzberg, northwest of Krautmoos, and southeast of the Mischenirwiese and north of the Steinwand. Photo below the map shows the study area as viewed from Mount Kampl to the southwest. The hilly area with dense forest and grassland consists of Jurassic cherty sediments with incorporated mass flows and slides of Hallstatt Limestones. The contact between matrix and blocks or complete sections is visible only in areas with steeper slopes or valleys, or anthropogenic excavations.

Quaternary deposits, but in rare cases some relics of the red nodular limestones of the Adnet Formation occur in the grassland below the dark-gray bedded radiolarite succession. Lower to Middle Jurassic condensed red limestones are well preserved on the southeastern slope of Mount Kampl on top of the Norian/ Rhaetian lagoonal Dachstein Limestone. This series represents the northeastern part of the syncline structure between the Steinwand and Mount Kampl.

The lowermost part of the radiolarite succession (sample BMW-28, Fig. 4) outcrops near the entrance of the valley and yielded the oldest assemblage, whereas the youngest part is preserved in the core of the syncline (sample BMW-35, Fig. 4). The thickness of this black radiolarite succession is nearly 100 meters. Intercalated mass-flow deposits are missing in contrast to equivalent successions to the east. Radiolarian dating proves a continuous radiolarite deposition from Bathonian to the Oxfordian. At the end of the valley, near a spring, a small outcrop of gray bioturbated cherty limestones yielded the youngest radiolarians in this area (sample BMW-33). This clearly demonstrates that the radiolarite succession in the valley is separated from the area of the Mischenirwiese by a young fault.

Area between Krautmoos and Mischenirwiese.-The area northwest of Krautmoos (Fig. 3) is characterized by a thick succession of mass-flow deposits with intercalated radiolarite matrix. In a few outcrops, below and between the amalgamated 

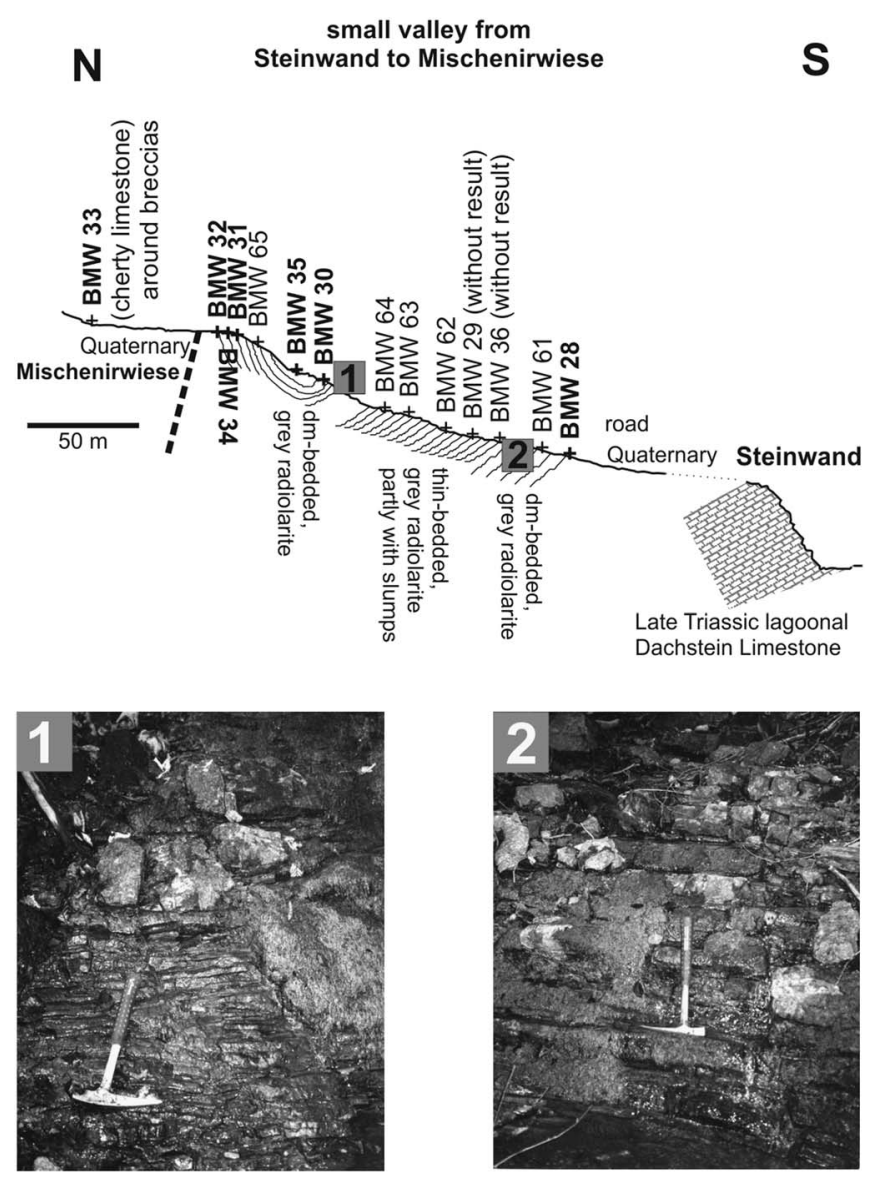

Figure 4. Cross-section in the small valley from Steinwand to Mischenirwiese, with the location of studied samples. Pictures 1 and 2 are details of the radiolaritic facies at lower (uppermost Bajocian-lower Bathonian) and upper (Oxfordian-? Kimmeridgian) part, respectively.

mass-flows matrix, radiolarians are well preserved, mainly in gray massive radiolarites. Two productive samples were taken, one from below the first mass flow (a massive dark-gray radiolarite, BMW-26) and another derived from the higher part of the mass-flow succession (sample BMW-13c).

The components of the different mass-flow deposits consist exclusively of different (gray and red) Hallstatt Limestone components, predominantly of Late Triassic age. Whereas in the lower part of the succession (near the top of the "Krautmoos hill") the size of the components does not exceed a few decimeters (e.g., near the sample BMW-26), the component size increases upsection. The succession is finally topped by slide blocks of half a kilometer in size, also near Krautmoos. Interestingly in these higher mass-flow deposits radiolarite components occur in addition to the Hallstatt Limestone clasts.

\section{Methods}

Samples were selected by examination of thin sections for wellpreserved radiolarians. Radiolarite samples were crushed to fragments $<2-3 \mathrm{~cm}$ in size and placed in 1-liter plastic jars with concentrated hydrochloric acid (32\%) until the reaction ceased and all the exposed calcium carbonate was dissolved. Then, the samples were rinsed and processed in the lab with diluted (4\%) hydrofluoric acid for a period of 24 hours to extract radiolarians. The samples were wet-sieved through $200-\mu \mathrm{m}$ and $63-\mu \mathrm{m}$ sieves and the residues were rinsed and dried in an oven at $40^{\circ} \mathrm{C}$. Eleven samples yielded diverse and relatively well-preserved assemblages (see Table 1 for species inventory), in part due to the low thermal overprint of these rocks (estimated by Conodont Color Alteration Index: CAI 1,0, see O'Dogherty and Gawlick, 2008).

Generic and suprageneric systematics used in this work follows De Wever et al. (2001) and O'Dogherty et al. (2009). Radiolarian taxonomy is based on the Middle Jurassic-Early Cretaceous catalogue of the InterRad Jurassic-Cretaceous Working Group (Baumgartner et al., 1995b) for 44 species. Age assignment is based on the Unitary Association Zones (UAZ) established by Baumgartner et al. (1995a), but in order to increase the resolution of this zonation a significant quantity of supplementary species (75 described species and 33 in open nomenclature) are introduced.

\section{Systematic paleontology}

Class Radiolaria Müller, 1858

Subclass Polycystina Ehrenberg, 1838

Order Nassellaria Ehrenberg, 1876

Monocyrtids

Family Poulpidae De Wever, 1981

Genus Saitoum Pessagno, 1977a

Type species.—Saitoum pagei Pessagno, 1977a.

Occurrence.-Lower Pliensbachian to upper Barremian.

Saitoum pagei Pessagno, 1977a

Figure 12.1

1977a Saitoum pagei Pessagno, p. 98, pl. 12, figs. 11-14.

2003 Saitoum pagei; Dumitrica and Zügel, p. 28, figs. 16A-B.

2003 Saitoum pagei; Suzuki and Gawlick, p. 175, fig. 5.38.

2006 Saitoum pagei; O'Dogherty et al., p. 458, pl. 11, figs. 6-8. [See for complete synonymy]

Saitoum trichylum De Wever, 1981

Figure 12.2-12.3

1981 Saitoum trichylum De Wever, p. 11, pl. 1, figs. 5-8.

1995b Saitoum trichylum; Baumgartner et al., p. 488, pl. 3021, figs. 1-6. [See for complete synonymy]

2002 Saitoum trichylum; Beccaro et al., pl. 2, fig. 14.

2003 Saitoum trichylum; Suzuki and Gawlick, p. 176, fig. 5.37.

'Dicyrtids'

Family Gongylothoracidae Bak, 1999

Genus Gongylothorax Foreman, 1968

Type species.—Dicolocapsa verbeeki Tan, 1927. 


\section{Kimmeridgian to Tithonian}

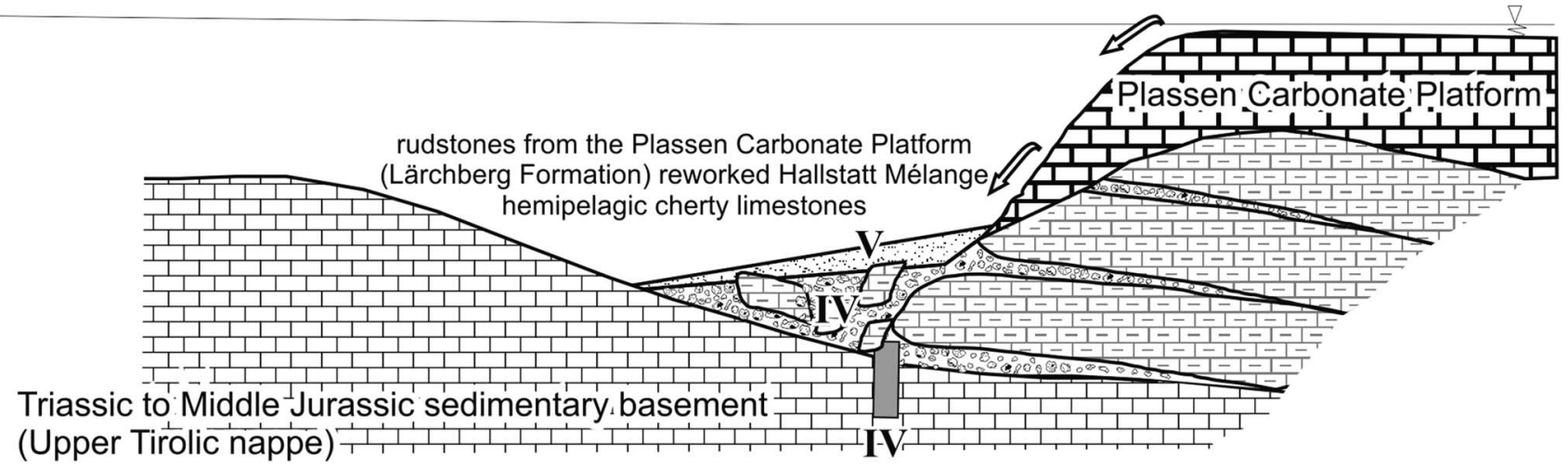

radiolarite section Mischenirwiese

\section{Oxfordian/Kimmeridgian boundary}

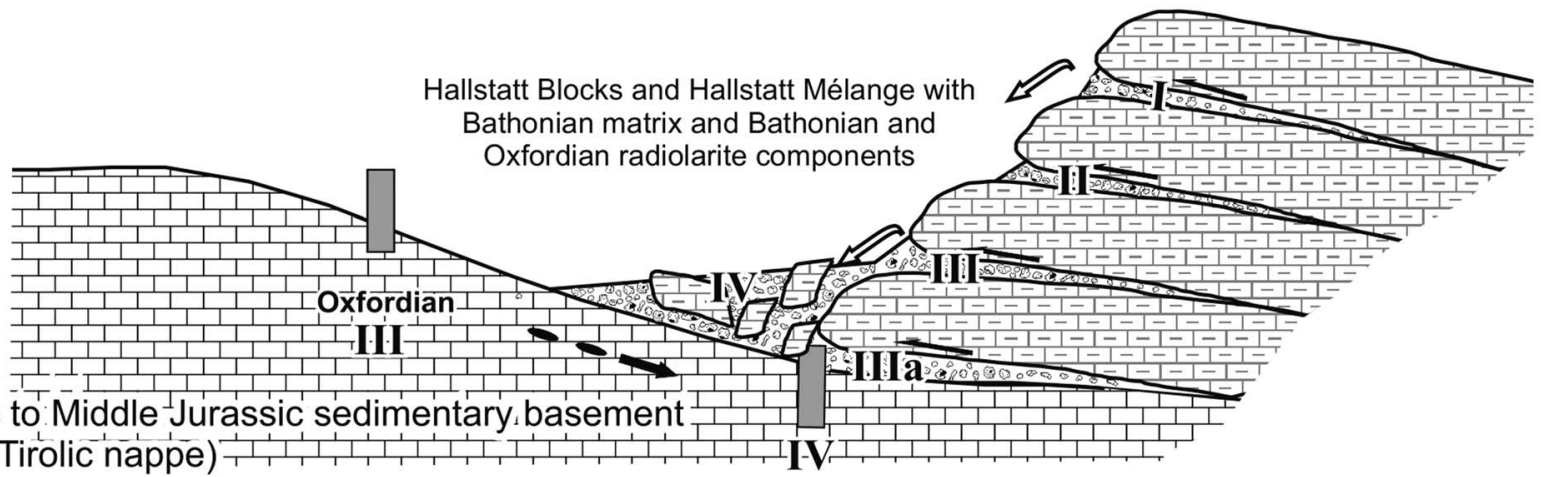

radiolarite section Mischenirwiese

\section{Bathonian to Oxfordian}

radiolarite section Mischenirwiese

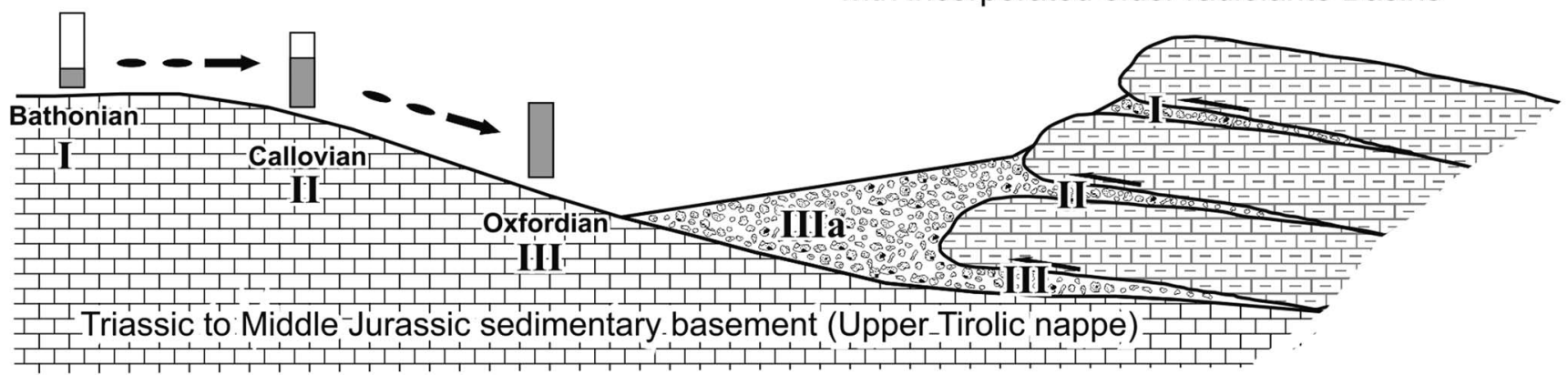

Figure 5. Reconstruction of the geologic evolution from Bathonian to Tithonian times. The radiolarite section Mischenirwiese is a complete radiolarite succession, reworked material from the advancing nappe stack, which consists of different Hallstatt imbricates, is missing. In Oxfordian times the succession was overridden by the Hallstatt mélange, which formed contemporaneously more to the south as indicated by the different radiolarite matrix ages. In KimmeridgianTithonian times this tectonic nappe stack was sealed by the Plassen Carbonate Platform sensu latu (Lärchberg Formation; see Figure 2). 
Table 1. Chart showing radiolarian taxa occurrences in studied samples; only absence or presence is noted. Solid circles indicate figured specimens; stars indicate unfigured specimens.

\begin{tabular}{|c|c|c|c|c|c|c|c|c|c|c|c|c|c|}
\hline \multirow{2}{*}{$\begin{array}{l}\text { Locality } \\
\text { Sample Number }\end{array}$} & \multirow{2}{*}{$\begin{array}{l}\text { EW } \\
158\end{array}$} & & & & & & IW & & & & & & \\
\hline & & 28 & 32 & 34 & 31 & 30 & 35 & 33 & 26 & $13 b$ & $13 \mathrm{c}$ & UAZ & Figure \\
\hline Arcanicapsa funatoensis & - & & & $\bullet$ & & & & & - & - & $\bullet$ & $(3-11)$ & $10.5-10.16$ \\
\hline Arcanicapsa sp. aff. A. funatoensis & & - & & & & & & & & & & & $11.27-11.28$ \\
\hline Arcanicapsa exquisita & & & $\bullet$ & & & & & & & & & & 10.3 \\
\hline Arcanicapsa sp. A & & & & & & & & & & • & & & 10.4 \\
\hline Arcanicapsa undulata & & & • & • & & & & • & & & & & $10.20-10.22$ \\
\hline Archaeodictyomitra sp. aff. A. exigua & & $\bullet$ & & & & & & & & • & & & $7.18-7.20$ \\
\hline Archaeodictyomitra sp. cf. A. annulata & & & & & & - & & & & & & & 7.21 \\
\hline Archaeodictyomitra minoensis & & & & & & • & $\bullet$ & $?$ & & & & $(9-12)$ & $7.40-7.42$ \\
\hline Archaeodictyomitra praeapiarium & • & & & & & & & & $\bullet$ & & & & $7.36-7.37$ \\
\hline Archaeodictyomitra prisca & & & & & & & & & & & • & & $7.43-7.45$ \\
\hline Archaeodictyomitra publica & & & & & & & & & & & $\bullet$ & & 7.17 \\
\hline Archaeodictyomitra rigida & & $\bullet$ & & & & $\bullet$ & $\bullet$ & & & & & & $7.33-7.35$ \\
\hline Archaeodictyomitra spelae & & & & & & & & & • & & & & 7.48 \\
\hline Archaeodictyomitra tyaughtonensis & & & & & & & $\bullet$ & $\bullet$ & & & & & $7.38-7.39$ \\
\hline Archaeodictyomitra whalenae & & & & & $\bullet$ & & & & & & • & & $7.46-7.47$ \\
\hline Belleza decora & & & & & & & & & & & - & $(4-7)$ & 8.53 \\
\hline Thanarla patricki gr. & $\bullet$ & & & & & & $\bullet$ & $\bullet$ & & • & • & & $7.22-7.32$ \\
\hline Canoptum krahsteinense & & & & & & & & & & • & & & 6.33 \\
\hline Cinguloturris carpatica & & & & & & & & & & & $\bullet$ & $(7-11)$ & $6.29-6.30$ \\
\hline Cinguloturris getsensis & & & & & & • & & & • & & & & $6.31-6.32$ \\
\hline Cinguloturris latiannulata & & & & & & & & & - & & & & 6.34 \\
\hline Theocapsomella cordis & & & & & & & & & & $\bullet$ & & $(5-8)$ & 11.46 \\
\hline Theocapsomella medvednicensis & & & $\bullet$ & & & & & & & & & & 11.47 \\
\hline Eucyrtidiellum nodosum & & & & & & & & & & & $\bullet$ & $(3-10)$ & $8.21-8.23$ \\
\hline Eucyrtidiellum ptyctum & & & & & & & ㅁ & & & & • & $(5-11)$ & 8.20 \\
\hline Eucyrtidiellum pustulatum & & & & & & & & $\bullet$ & & • & & $(5-8)$ & $8.26-8.27$ \\
\hline Eucyrtidiellum pyramis & & & & & & & & $\bullet$ & & & & $(12-13)$ & 8.19 \\
\hline Eucyrtidiellum unumaense & & $\bullet$ & & & $\bullet$ & & & & & & & $(3-8)$ & $8.24-8.25$ \\
\hline Spinosicapsa basilica & & & & & & & & & & & • & & 8.28 \\
\hline Spinosicapsa sp. cf. S. triacantha & & & & & & & & & & & - & & 8.29 \\
\hline Spinosicapsa lata & & & & & & & & $\bullet$ & & & & & 8.30 \\
\hline Spinosicapsa spinosa & & & & & & & & & & & - & $(8-13)$ & 8.31 \\
\hline Gongylothorax favosus & & & $\bullet$ & $\bullet$ & & $\bullet$ & & $\bullet$ & & & & $(8-10)$ & $9.39-9.42$ \\
\hline Gongylothorax marmoris & & & $\bullet$ & & & & & & & & & & $11.1-11.3$ \\
\hline Gongylothorax sp. A & & & & $\bullet$ & & & & & & & & & 9.38 \\
\hline Kilinora? oblongula & & & $\bullet$ & & & & & & & & & $(6-8)$ & 9.9 \\
\hline Kilinora? sp. aff. K. oblongula & - & & $\bullet$ & & & & & & & & & & $10.25-10.26$ \\
\hline Hsuum arabicum & & & & & & & $\bullet$ & & & & & & 7.54 \\
\hline Hsuum obispoense & & & & $\bullet$ & & & & & & & & & 7.55 \\
\hline Parahsuum sp. aff. P. probosum & & $\bullet$ & & & & & & & & & & & 7.61 \\
\hline Parahsuum snowshoense & & & & & $\bullet$ & & & & & & & & 7.50 \\
\hline Parahsuum sp. aff. P. snowshoense & & & & & & & & & & & • & & 7.51 \\
\hline Parahsuum carpathicum & & & & & & & & & & & • & & $7.52-7.53$ \\
\hline Parahsuum sp. 1 & & & & $\bullet$ & & & & & & & & & 7.49 \\
\hline Parahsuum sp. 2 & & & & & $\bullet$ & & & & & & • & & $7.56-7.60$ \\
\hline Parahsuum sp. 3 & & & & & & & & & & & - & & $7.62-7.63$ \\
\hline Semihsuum amabile & - & & & & & & & & - & & & $(4-7)$ & $7.15-7.16$ \\
\hline Transhsuum brevicostatum & & $\bullet$ & & $\bullet$ & & & & & $\bullet$ & & & $(3-11)$ & $8.1-8.3$ \\
\hline Transhsuum maxwelli & $\bullet$ & $\bullet$ & & & & & $\bullet$ & & $\bullet$ & & - & & $8.4-8.8,8.10-8.18$ \\
\hline Transhsuum sp. E & & $\bullet$ & & & & & & & & & & & 8.9 \\
\hline Japonocapsa sp. A & & & & & & & & & & • & & & 11.4 \\
\hline Striatojaponocapsa conexa & • & & $\bullet$ & & & & & & & & & $(4-7)$ & $11.5-11.7$ \\
\hline Striatojaponocapsa riri & & & • & & & & & & & & & & $11.8-11.9$ \\
\hline Striatojaponocapsa synconexa & $\bullet$ & & & & & & & & $\bullet$ & & & & $11.10-11.13$ \\
\hline Striatojaponocapsa spp. & & & • & $\bullet$ & & & & & & & & & $11.14-11.16$ \\
\hline Striatojaponocapsa? spp. & • & & • & & & & & & & • & & & $11.17-11.20$ \\
\hline Crococapsa tansinhoki & & & & & & & $\bullet$ & $\bullet$ & & & & & $8.46-8.47$ \\
\hline Crococapsa sp. aff. C. tansinhoki & & & & & & & & & & & - & & $9.17-9.18$ \\
\hline Crococapsa sp. aff. C. truncata & & - & & & & & & & & & & & 8.48 \\
\hline Crococapsa sp. A & & & & & & & & & & & - & & $8.49-8.52$ \\
\hline Crococapsa sp. B & & & & & & & $\bullet$ & & & & & & 8.45 \\
\hline Crococapsa sp. C & • & & & & & & & & & & & & $8.43-8.44$ \\
\hline Doliocapsa keni & & & & - & & & & & & & & & 7.4 \\
\hline Doliocapsa matsuokai & & & & & • & & & & & - & & & $8.33-8.34$ \\
\hline Doliocapsa planata & & & & $\bullet$ & & & & & & & • & & $8.35-8.37$ \\
\hline Hiscocapsa kodrai & & & & & & & & & & - & & & 9.53 \\
\hline Praewilliriedellum convexum & & • & & & & & & & & • & & $(1-11)$ & $9.27-9.28$ \\
\hline Praewilliriedellum robustum & $?$ & & & $\bullet$ & • & & & & & & & $(5-7)$ & $10.45 ? 10.46-10.47$ \\
\hline Quarkus japonicus & & & & & $\bullet$ & & & & & • & & $(3-8)$ & $9.25-9.26$ \\
\hline Quarkus madstonensis & & & & & $\bullet$ & & & & & & & & 9.24 \\
\hline Olanda sp. & & $\bullet$ & & & & & & & & & & & 7.14 \\
\hline Caneta hsui & & & & & & & $\bullet$ & & & & & & 6.25 \\
\hline Eoxitus baloghi & & - & & & & & & & $\bullet$ & • & & & $6.7-6.11$ \\
\hline Eoxitus dhimenaensis & & & $\bullet$ & & & & & & & & - & $(3-11)$ & $6.15-6.16$ \\
\hline Eoxitus hungaricus & - & $\bullet$ & & & $\bullet$ & & & & & 口 & & $(3-8)$ & $6.1-6.6$ \\
\hline Eoxitus? brevis & & & & & & & & & $\bullet$ & & & & 7.9 \\
\hline Eoxitus? sp. A & & & & & - & & & & & & & & 6.17 \\
\hline Eoxitus? sp. B & & & & & $\bullet$ & & & & & & & & 6.28 \\
\hline Takemuraella japonica & & & & & & & & & & - & • & & $6.49-6.50$ \\
\hline
\end{tabular}


Table 1. (Continued)

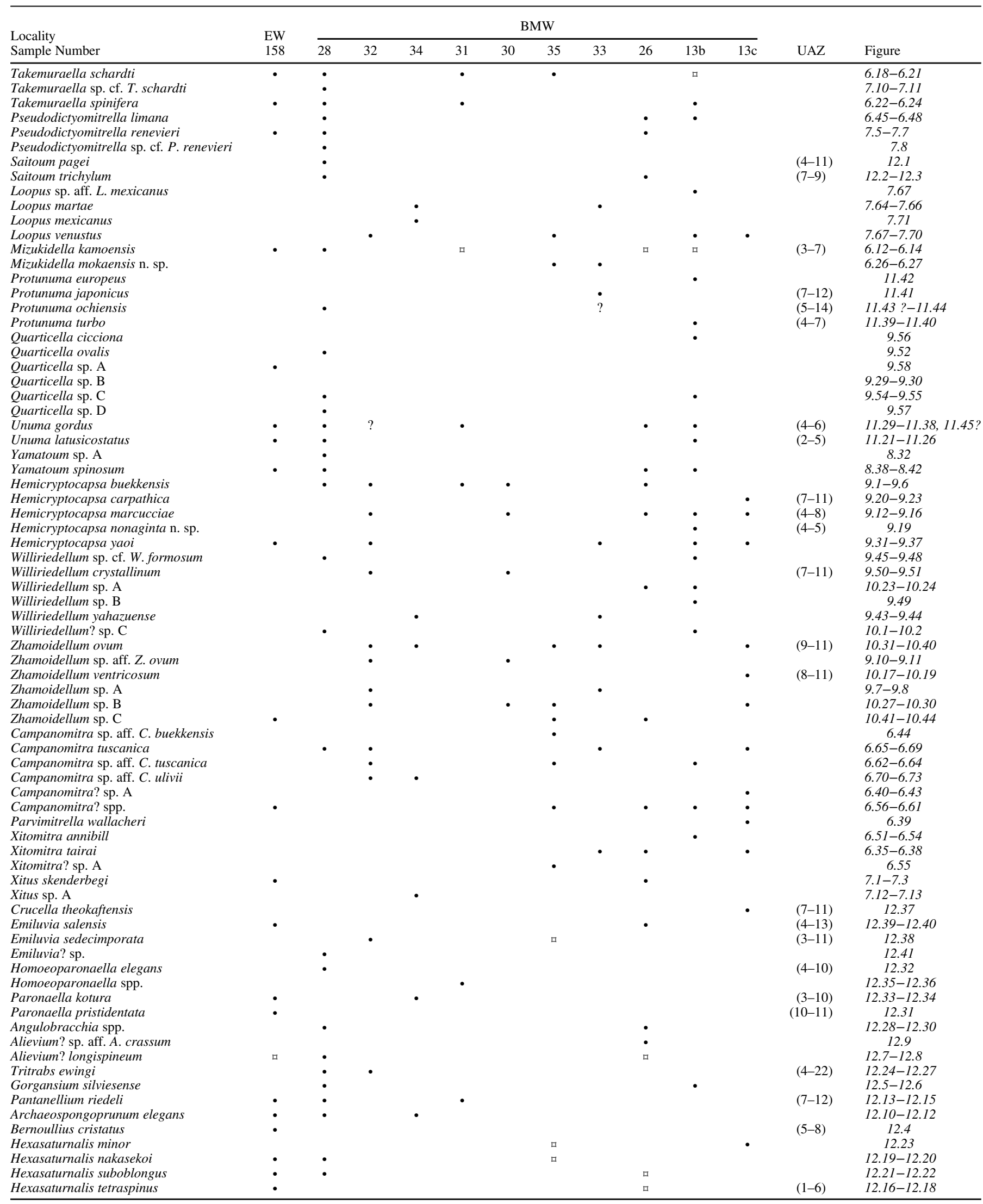




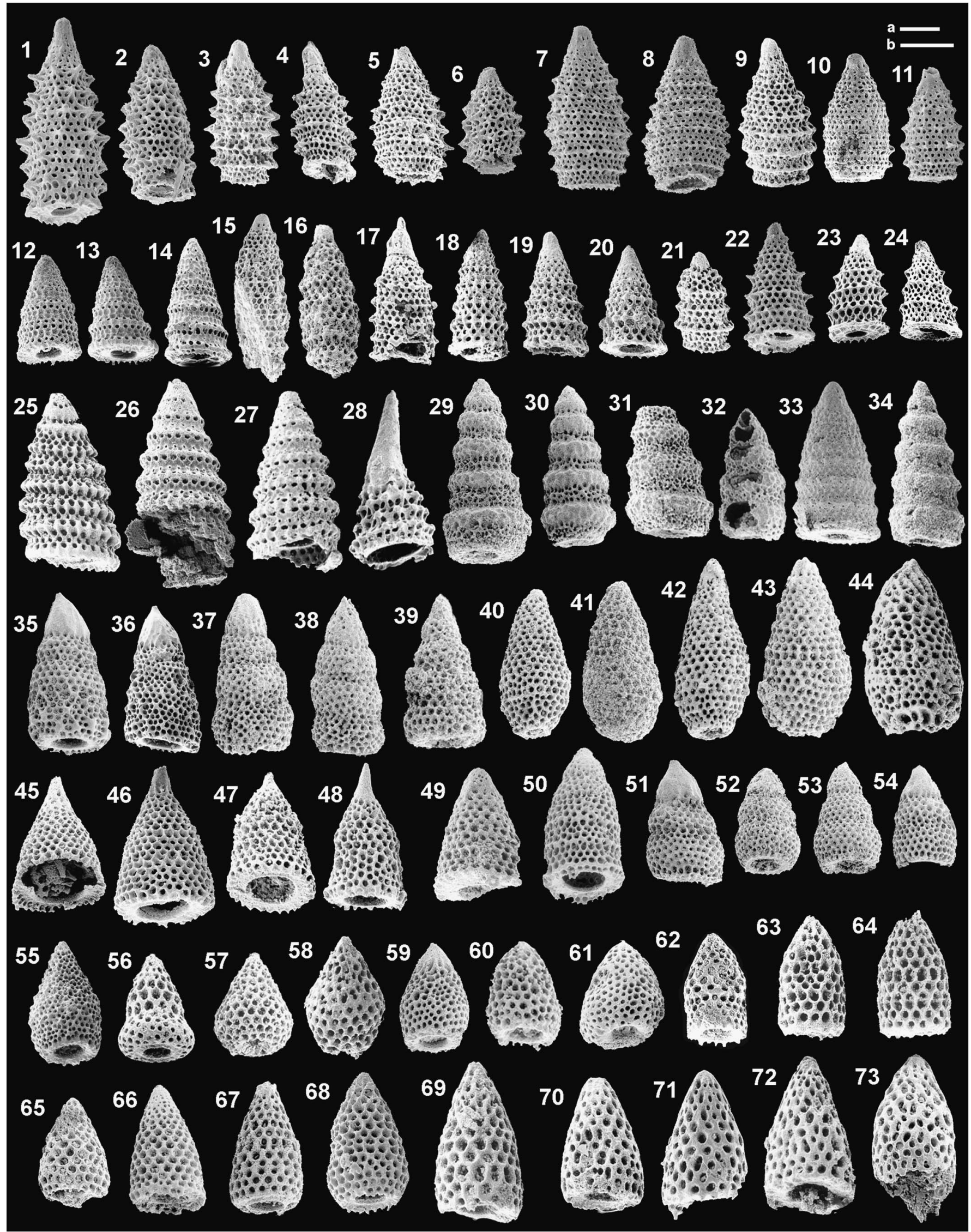


Occurrence.-Upper Bajocian to upper Maastrichtian.

Gongylothorax favosus Dumitrica, 1970

Figure 9.39-9.42

1970 Gongylothorax favosus Dumitrica, p. 56, pl. 1, figs. 1a-c, 2.

1995b Gongylothorax favosus; Baumgartner et al., p. 230, pl. 6131, figs. 1-7.

2003 Gongylothorax favosus; Suzuki and Gawlick, p. 205, fig. 6.96. [See for complete synonymy]

2009 Gongylothorax favosus favosus; Suzuki and Gawlick, p. 180, fig. 5.31A-C, 32A-B; fig. 6.21A-B. [See for complete synonymy]

Gongylothorax marmoris Kiessling in Kiessling and Zeiss, 1992 Figure 11.1-11.3

1992 Gongylothorax (?) marmoris Kiessling in Kiessling and Zeiss, p. 190, pl. 2, figs. 8-10.

2003 Gongylothorax cf. marmoris; Suzuki and Gawlick, fig. 5.47.

Remarks.-Originally this species was questionally attributed to Gongylothorax because the internal structure of the cephalis was not observed, but otherwise all characteristics match with the diagnosis of the genus.

\section{Gongylothorax sp. A}

Figure 9.38

Remarks.-The ridges around the hexagonal areas are more elevated in relief than the pores commonly observed in Gongylothorax (see Gongylothorax sp. aff. G. favosus in Baumgartner et al., 1995b, p. $232=$ Gongylothorax favosus oviformis Suzuki and Gawlick 2009, p. 180, fig. 5.33A-34C; fig. 6.22A-26B).

Genus Kilinora Hull, 1997

Type species.—Stylocapsa? spiralis Matsuoka, 1982.
Occurrence.-Lower Bathonian to middle Callovian.

Kilinora? oblongula (Kocher in Baumgartner et al., 1980) Figure 9.9

1980 Stylocapsa oblongula Kocher in Baumgartner et al., p. 62, pl. 6, fig. 1.

2006 Kilinora (?) oblongula; O'Dogherty et al., p. 443, pl. 8, figs. 25-29. [See for complete synonymy]

2007b Stylocapsa oblongula; Gawlick et al., fig. 17.25.

2013 Kilinora (?) oblongula; Chiari et al., fig. 13c.

Remarks.-According to O'Dogherty et al., 2006, the genus is queried because this species does not exhibit a linear arrangement of pores, and clearly lacks costae. It may belong to a new genus.

Kilinora? sp. aff. K. oblongula (Kocher in Baumgartner et al., 1980) Figure 10.25-10.26

Remarks.-This form strongly resembles Kilinora (?) oblongula as illustrated by O'Dogherty et al., 2006 on pl. 8, figures 25 -26 , but possesses a somewhat pointed basal appendage.

\author{
'Multicyrtids' \\ Family Diacanthocapsidae O’Dogherty, 1994
}

Genus Theocapsomella O'Dogherty, Goričan, and Dumitrica in O'Dogherty et al., 2006.

Type species.—Theocapsomma cordis Kocher, 1981.

Remarks.-This genus was misspelled as Theocapsommella in all descriptions of the species originally included under this genus (O'Dogherty et al., 2006). According to ICZN Art. 24.2.3, the selection of the correct original spelling should be Theocapsomella. This was the original name followed by the expression n. gen. (O’Dogherty et al., 2006, p. 454) and was also fixed as the correct original spelling by the first reviser (O’Dogherty et al., 2009).

Figure 6. Scale bars (a, b) measure $50 \mu \mathrm{m}$; each radiolarian scanning micrograph is designated by sample and specimen number, followed by the corresponding scale. (1-6) Eoxitus hungaricus Kozur, sample BMW-28 (1, 073/a; 2, 072/a; 6, 043/a); sample EW-158 (3, 005/a; 5, 017/a); sample BMW-31 (4, 008/a). (7-11) Eoxitus baloghi Kozur, sample BMW-28 (7, 045/a; 8, 070/a; 11, 061/a); sample BMW-26 (9, 011/a); sample BMW-13b (10, 017/a). (12-14) Mizukidella kamoensis (Mizutani and Kido), sample BMW-28 (12, 064/a); sample EW-158 (13, 009/a; 14, 015/a). (15-16) Eoxitus dhimenaensis (Baumgartner), sample BMW-32 (15, 077/a); sample BMW-13c (16, 052/a). (17) Eoxitus? sp. A, sample BMW-31 (17, 011/a). (18-21) Takemuraella schardti (O'Dogherty, Goričan, and Dumitrica in O'Dogherty et al.) sample BMW-35 (18, 006/a); sample BMW-26 (19, 008/a; 20, 016/a; 21, 055/a). (22-24) Takemuraella spinifera (Takemura), sample BMW-28 (22, 060/a); sample BMW-31 (23, 018/a); sample EW-158 (24, 060/a). (25) Caneta hsui (Pessagno), sample BMW-35 (25, 015/a). (26-27) Mizukidella mokaensis n. sp., sample BMW-33 (26, 004/b, paratype); sample BMW-35 (27, 001/a, holotype). (28) Eoxitus? sp. B, sample BMW-31 (28, 028/b). (29-30) Cinguloturris carpatica Dumitrica in Dumitrica and Mello, sample BMW-13c (29, 026/a; 30, 018/a). (31-32) Cinguloturris getsensis O'Dogherty, Goričan, and Dumitrica in O'Dogherty et al., sample BMW-26 (31, 025/a); sample BMW-30 (32, 001/b). (33) Canoptum krahsteinense (Suzuki and Gawlick in Gawlick et al.), sample BMW-13b (33, 19/b). (34) Cinguloturris latiannulata (Grill and Kozur), sample BMW-26 (34, 010/b). (35-38) Xitomitra tairai (Aita), sample BMW-13c (35, 055/a; 37, 043/a; 38, 070/a); sample BMW-33 (36, 001/a). (39) Parvimitrella wallacheri (Grill and Kozur), sample BMW-13c (39, 036/b). (40-43) Campanomitra? sp. A, sample BMW-13c (40, 021/a; 41, 054/a; 42, 010/a; 43, 030/a). (44) Campanomitra sp. aff. C. buekkensis (Grill and Kozur), sample BMW-35 (44,012/a). (45-48) Pseudodictyomitrella limana (Cortese), sample BMW-13b (45, 032/b); sample BMW-28 (46, 014/b); sample BMW-26 (47, 048/b; 48, 039/b). (49-50) Takemuraella japonica (Takemura), sample BMW-13b (49, 013/b); sample BMW-13c (50, 034/a). (51-54) Xitomitra annibill (Kocher), sample BMW-13c (51, 088/a; 52, 028/a; 53, 044/a; 54, 042/a). (55) Xitomitra? sp. A, sample BMW-35 (55, 020/a). (56-61) Campanomitra? spp., sample BMW-26 (56, 054/b); sample EW-158 (57, 026/b); sample BMW-35 (58, 026/a); sample BMW-13c (59, 045/a; 60, 092/b); sample BMW-13b (61, 050/b). (62-64) Campanomitra sp. aff. C. tuscanica (Chiari, Cortese, and Marcucci in Chiari et al.), sample BMW-32 (62, 041/b); sample BMW-35 (63, 023/b); sample BMW-13b (64, 012/b). (65-69) Campanomitra tuscanica (Chiari, Cortese, and Marcucci in Chiari et al.), sample BMW-32 (65, 030/a); sample BMW-13c (66, 126/a; 67, 077/b); sample BMW-28 (68, 001/a); sample BMW-33 (69, 008/b). (70-73) Campanomitra sp. aff. C. ulivii (Chiari, Cortese, and Marcucci in Chiari et al.), sample BMW-34 (70, 014/b; 71, 012/b; 72, 034/b); sample BMW-32 (73, 102/b). 

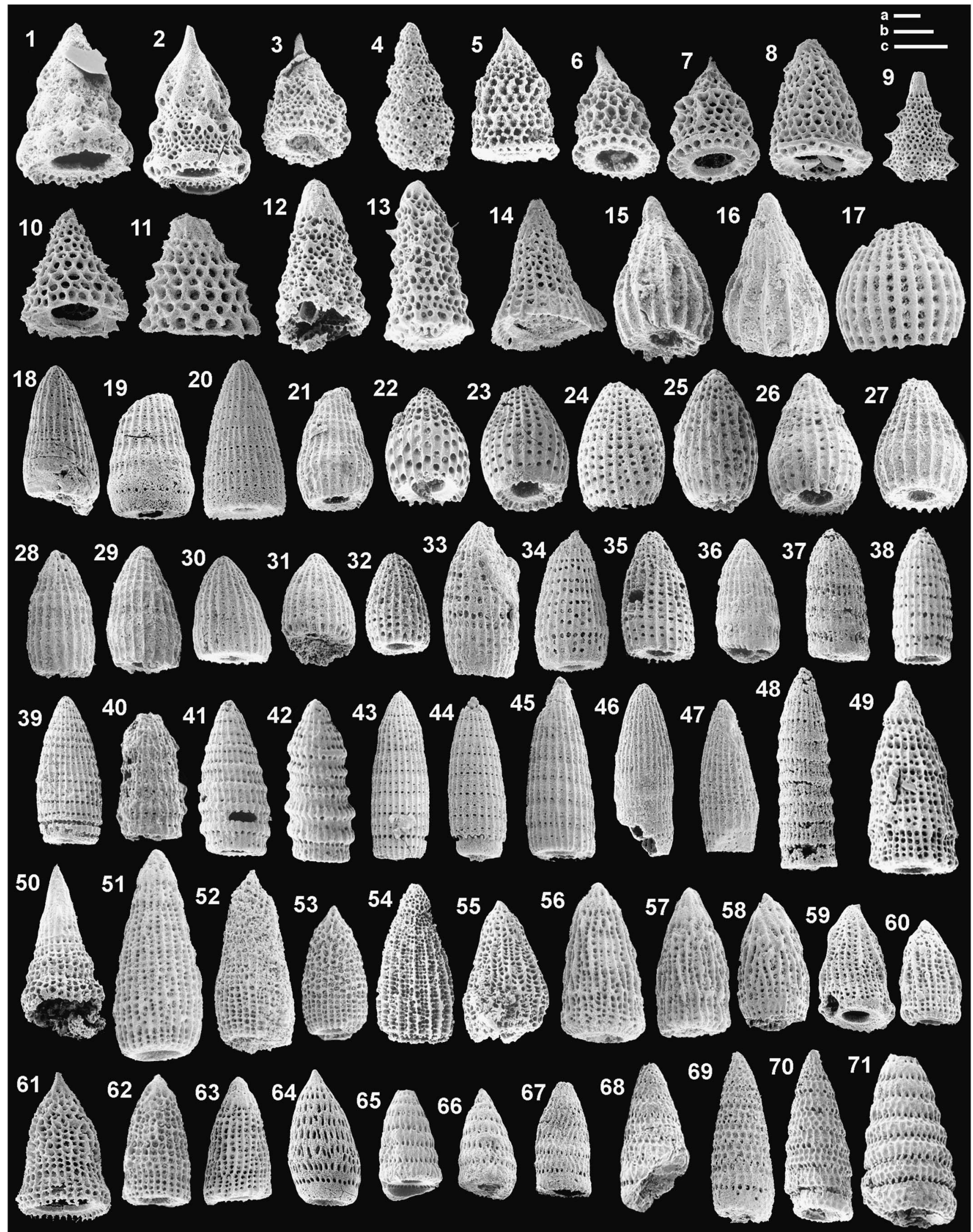
In the original description (O'Dogherty et al., 2006), Theocapsomella included also some four-segmented nassellarians (e.g., Stichocapsa himedaruma Aita). On the other hand, a phylogenetic relationship of Stichocapsa himedaruma Aita and Stichocapsa convexa Yao was also assumed (see O'Dogherty et al., 2006, p. 441). New findings are in favor of the latter opinion (see Šegvić et al., 2014, pl. 1, figs. 21, 22).

Occurrence.-Lower Bathonian to lower Berriasian.

Theocapsomella cordis (Kocher in Baumgartner et al., 1980) Figure 11.46

1980 Stylocapsa cordis Kocher in Baumgartner et al., p. 62, pl. 6, fig. 1.

2006 Theocapsommella cordis; O'Dogherty et al., p. 443, pl. 8, figs. 25-29. [See for complete synonymy]

2009 Theocapsomma cordis; Suzuki and Gawlick, p. 181, figs. 5.37A-B, 6.28A-B.

2013 Theocapsomella cordis; Chiari et al., fig. 14e.

Theocapsomella medvednicensis (Goričan in Halamić et al., 1999) Figure 11.47

1999 Theocapsomma medvednicensis Goričan in Halamić et al., p. 37, pl. 1, figs. 12-16.

2003 Theocapsomma medvednicensis; Suzuki and Gawlick, p. 206, fig. 6.87 .

2006 Theocapsommella medvednicensis (Goričan); O’Dogherty et al., p. 456, pl. 8, figs. 30, 33-37. [See for complete synonymy]

2008 Theocapsomma sp. aff. T. medvednicensis; Baumgartner et al., pl. 4, fig. 10.

2009 Theocapsomma medvednicensis; Suzuki and Gawlick, p. 181, figs. 5.38A-B.

Family Minocapsidae new family
Type genus.—Minocapsa Matsuoka, 1991a.

Other genera.-Crococapsa new genus; Doliocapsa new genus; Hemicryptocephalis Li, 1988; Hiscocapsa O'Dogherty, 1994; Praewilliriedellum Kozur, 1984 (syn. Hemicryptocephalis); and Quarkus Pessagno, Blome, and Hull in Pessagno et al., 1993.

Diagnosis.-Pyriform to ovoidal shell consisting in usually four segments. Last segment with or without an aperture. Lattice shell composed of small rounded to large polygonal pore frames.

Etymology.-After type genus.

Occurrence.—Early Pliensbachian to Middle Albian.

Remarks.-This family is erected for genera included in the unnamed family pro Stichocapsidae used by O'Dogherty et al., 2009. The genus Aitaum Pessagno and Hull, 2002 is an exception because it has a multisegmented test composed of three to four post-abdominal segments. Aitaum has more affinity with the genus Lantus Yeh, 1987a and other eucyrtidiid-type nassellarians.

\section{Genus Crococapsa new genus}

Type species.—Sethocapsa hexagona Hori, 1999.

Other species.-Cyrtocapsa asseni Tan, 1927; Minocapsa aitai Hull, 1997; Minocapsa truncata Wu, 2000; Minocapsa? tansinhoki Hull, 1997; Sethocapsa accincta Steiger, 1992; Sethocapsa horokanaiensis Kawabata, 1988; Sethocapsa kitoi Jud, 1994; Sethocapsa lagenaria Wu and Li, 1982; Sethocapsa pseudouterculus Aita in Aita and Okada, 1986; Sethocapsa? subcrassitestata Aita in Aita and Okada, 1986; Sethocapsa? zweilii Jud, 1994; Sethocapsa hashimotoi Tumanda, 1989; Theocapsa simplex Tan, 1927; and Theocapsa uterculus Parona, 1890.

Figure 7. Scale bars $(\mathrm{a}, \mathrm{b}, \mathrm{c})$ measure $50 \mu \mathrm{m}$; each radiolarian scanning micrograph is designated by sample and specimen number, followed by the corresponding scale. (1-3) Xitus skenderbegi (Chiari, Marcucci, and Prela), sample BMW-26 (1, 009/c; 3, 066/c); sample EW-158 (2, 014/c). (4) Doliocapsa keni (Kocher), sample BMW-34 (4,035/c). (5-7) Pseudodictyomitrella renevieri O’Dogherty, Goričan, and Dumitrica in O'Dogherty et al., sample EW-158 (5, 055/c); sample BMW-26 (6, 036/c); sample BMW-28 (7, 003/c). (8) Pseudodictyomitrella sp. cf. P. renevieri O'Dogherty, Goričan, and Dumitrica in O'Dogherty et al., sample BMW-28 (8, 046/c). (9) Eoxitus? brevis Kozur, sample BMW-26 (9, 038/b). (10-11) Takemuraella sp. cf. T. schardti (O'Dogherty, Goričan, and Dumitrica in O'Dogherty et al.), sample BMW-28 (10, 063/c; 11, 053/c). (12-13) Xitus sp. A, sample BMW-34 (12, 008/c; 13, 005/c). (14) Olanda sp., sample BMW-28 (14, 067/c). (15-16) Semihsuum amabile (Aita), sample BMW-26 (15, 070/c); sample EW-158 (16, 007/c). (17) Archaeodictyomitra publica (Hull), sample BMW-13c (17, 099/c). (18-20) Archaeodictyomitra sp. aff. A. exigua Blome, sample BMW-13b (18, 013/c; 19, 028/c); sample BMW-28 (20, 062/c). (21) Archaeodictyomitra sp. cf. A. annulata Kozur and Mostler in Grill and Kozur, sample BMW-30 (21, 59/c). (22-32) Thanarla patricki gr. (Kocher), sample BMW-33 (22, 022/c); sample BMW-35 (23, 028/c; 24, 024/c; 25, 037/c; 26, 030/c; 29, 007/c; 32, 025/c); sample EW-158 (27, 063/c); sample BMW-13c (28, 129/c); sample BMW-13b (30, 004/c; 31, 022/c). (33-35) Archaeodictyomitra rigida Pessagno, sample BMW-30 (33, 039/c); sample BMW-28 (34, 042/c); sample BMW-35 (35, 021/c). (36-37) Archaeodictyomitra praeapiarium Cordey, sample BMW-26 (36, 024/b); sample EW-158 (37, 059/b). (38-39) Archaeodictyomitra tyaughtonensis Cordey, sample BMW-35 (38, 005/b; sample BMW-33 (39, 005/b). (40-42) Archaeodictyomitra minoensis (Mizutani), sample BMW-30 (40, 057/b); sample BMW-35 (41, 017/b; 42, 010/b). (43-45) Archaeodictyomitra prisca Kozur and Mostler in Grill and Kozur, sample BMW-13c (43, 075/b; 44, 051/b; 45, 017/b). (46-47) Archaeodictyomitra whalenae Kozur and Mostler in Grill and Kozur, BMW-31 (46, 026/b); sample BMW-13c (47, 049/b). (48) Archaeodictyomitra spelae Chiari, Cortese and Marcucci in Chiari et al., sample BMW-26 (48, 043/b). (49) Parahsuum sp. 1 sensu O'Dogherty et al., sample BMW-34 (49,006/c). (50) Parahsuum snowshoense (Pessagno and Whalen), sample BMW-31 (50, 021/b). (51) Parahsuum sp. aff. P. snowshoense (Pessagno and Whalen), sample BMW-13c (51, 031/b). (52-53) Parahsuum carpathicum Widz and De Wever, sample BMW-13c (52, 050/b; 53, 076/b). (54) Hsuum arabicum Dumitrica in Dumitrica et al., sample BMW-35 (54, 009/a). (55) Hsuum obispoense Pessagno, sample BMW-34 (55, 7/b). (56-60) Parahsuum sp. 2 sensu O'Dogherty et al., sample BMW-13c (56, 020/b; 57, 025/b; 58, 033/b); sample BMW-31 (59, 016/b; 60, 070/b). (61) Parahsuum sp. aff. P. probosum (Pessagno and Whalen), sample BMW-28 (61, 054/c). (62-63) Parahsuum sp. 3 sensu O'Dogherty et al., sample BMW-13c (62, 035/b; 63, 080/b). (64-66) Loopus martae Beccaro, sample BMW-34 (64, 003/b; 65, 009/b); sample BMW-33 (66, 007/b). (67-70) Loopus venustus (Chiari, Cortese, and Marcucci in Chiari et al.), sample BMW-13b (67, 001/b); sample BMW-32 (68, 009/c); sample BMW-13c (69, 082/c); sample BMW-35 (70, 011/c). (71) Loopus mexicanus (Hull), sample BMW-34 (71, 020/c). 


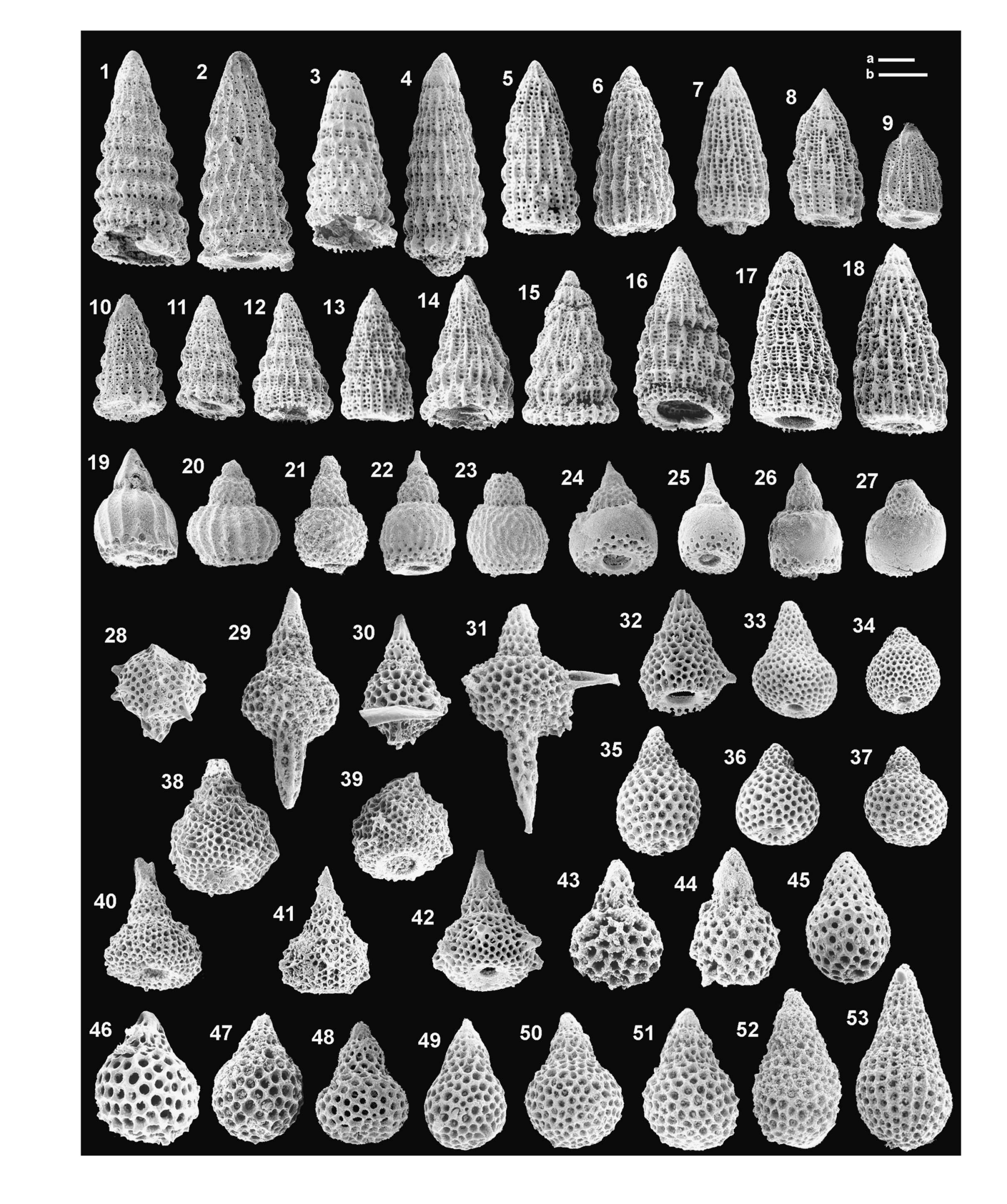


Diagnosis.-Tetracyrtid nassellarian having a globose postabdominal segment. Lattice meshwork with polygonal pore frames lacking nodes or tubercles on its surface. Closed antapically or having a discrete number of small pores grouped into a depression (see Sethocapsa uterculus in Baumgartner et al., 1995b, p. 505, pl. 5462, fig. 2). Collar and lumbar strictures slightly recognizable or indistinct externally. Large and globose postabdominal segment is well distinguished from the abdomen by a marked stricture.

Etymology.-From the latin croco (saffron) and capsa (box); feminine gender.

Occurrence.-Bathonian to middle Albian.

Remarks.-O'Dogherty (1994) erected the genus Hiscocapsa for a heterogeneous group of tetracyrtid nassellarians displaying a wide variety of pore frame arrangement. However, many species assigned originally to Hiscocapsa are clearly separated by lacking a distal aperture and bearing no tubercles on the surface.

Crococapsa n. gen. differs from Minocapsa Matsuoka, 1991a, by its distinct fourth segment, which is globose and well differentiated from the abdomen by a marked stricture. Crococapa n. gen. also differs from Lantus Yeh, 1987a by having only one postabdominal chamber.

\section{Crococapsa tansinhoki (Hull, 1997)}

Figure 8.46-8.47

1997 Minocapsa(?) tansinhoki Hull, p. 148, pl. 38, figs. 4, 6.

2006 Minocapsa(?) tansinhoki; O'Dogherty et al., 2006, p. 444, pl. 6, figs. 16, 17. [See for complete synonymy]

Remarks.-O'Dogherty et al. (2006, p. 444) first questioned the placement of this species to Minocapsa because the basal aperture was absent and the phylogentic relationship with early Jurassic representatives was not demonstrated. This species fits well in the general description of the new genus Crococapsa.

Crococapsa sp. aff. C. tansinhoki (Hull, 1997)

Figure 9.17-9.18

Remarks.-These forms differ from Hull's material by having smaller and more numerous pores on the abdomen.
Crococapsa sp. aff. C. truncata (Wu, 2000)

Figure 8.48

aff. 2000 Minocapsa truncata Wu, p. 304, pl. 2, figs. 1, 5-6.

Remarks.-This specimen differs from the Tibetan specimens by displaying a proportionally larger last segment.

Crococapsa sp. A

Figure 8.49-8.52

Remarks.-These specimens resemble Crococapsa sp. aff. C. truncata (Wu, 2000), but the proximal part of the shell is relatively longer and elongated.

\section{Crococapsa sp. B \\ Figure 8.45}

Remarks.-This specimen is closely related to other congeneric forms included in this paper, but differs by the well-marked polygonal pore frames on almost all the shell surface.

\section{Crococapsa sp. C}

Figure 8.43-8.44

Remarks.-These specimens are very close to Crococapsa tansinhoki but they display more cylindrical and smoother thorax.

\section{Genus Doliocapsa new genus}

Type species.-Stichomitra (?) stecki O'Dogherty, Goričan, and Dumitrica in O'Dogherty et al., 2006.

Other species.-Hiscocapsa acuta Hull, 1997; Hiscocapsa minuta Yeh, 2011; Hiscocapsa planata Wu, 2000 (syn. Stichocapsa magnipora); Sethocapsa lugeoni O'Dogherty, Goričan, and Dumitrica in O'Dogherty et al., 2006; Sethocapsa taukhaensis Kemkin and Taketani, 2004; Solenotryma keni Kocher, 1981; Stichocapsa magnipora Chiari, Marcucci, and Prela, 2002; Stichomitra doliolum Aita in Aita and Okada, 1986; and ?Quarticella hunzikeri O'Dogherty, Goričan, and Dumitrica in O'Dogherty et al., 2006.

Figure 8. Scale bars (a, b) measure $50 \mu \mathrm{m}$; each radiolarian scanning micrograph is designated by sample and specimen number, followed by the corresponding scale. (1-3) Transhsuum brevicostatum (Ožvoldová), sample BMW-26 (1, 013/b); sample BMW-28 (2, 068/a); sample BMW-34 (3, 002/a). (4-8, 10-18) Transhsuum maxwelli (Pessagno), BMW-13c (4, 083/a; 6, 021/a; 7, 048/a; 18, 024/a); sample BMW-35 (5, 003/a; 13, 027/a; 15, 004/a; 16, 002/a); sample BMW-26 (8, 015/a; 11, 060/a); sample BMW-28 (10, 071/a); sample EW-158 (12, 062/a; 14, 012/a; 17, 061/a). (9) Transhsuum sp. E sensu (Yao), sample BMW-28 (9, 066/a). (19) Eucyrtidiellum pyramis (Aita in Aita and Okada), sample BMW-33 (19, 019/b). (20) Eucyrtidiellum ptyctum (Riedel and Sanfilippo), BMW-13c (20, 139/b). (21-23) Eucyrtidiellum nodosum Wakita, sample BMW-13c (21, 074/b; 22, 005/b; 23, 134/b). (24-25) Eucyrtidiellum unumaense (Yao), sample BMW-28 (24, 017/b); sample BMW-31 (25, 045/b). (26-27) Eucyrtidiellum pustulatum Baumgartner, sample BMW-33 (26, 028/b); sample BMW-13b (27, 060/b). (28) Spinosicapsa basilica (Hull), sample BMW-13c (28, 064/a). (29) Spinosicapsa sp. cf. S. triacantha (Fischli), sample BMW-13c (29, 046/a). (30) Spinosicapsa lata (Yang), sample BMW-33 (30, 018/a). (31) Spinosicapsa spinosa (Ožvoldová), sample BMW-13c (31, 057/a). (32) Yamatoum sp. A, sample BMW-28 (32, 047/b). (33-34) Doliocapsa matsuokai (Yeh), sample BMW-13b (33, 049/b); sample BMW-31 (34, 048/b). (35-37) Doliocapsa planata (Wu), sample BMW-13c (35, 131/a; 37, 089/a); sample BMW-34 (36, 029/a). (38-42) Yamatoum spinosum (Takemura), sample EW-158 (38, 022/b); sample BMW-13b (39, 054/b); sample BMW-26 (40, 073/b; 41, 049/b); sample BMW-28 (42, 051/b). (43-44) Crococapsa sp. C, sample EW-158 (43,067/b; 44, 070/b). (45) Crococapsa sp. B, sample BMW-35 (45, 032/b). (46-47), Crococapsa tansinhoki (Hull), sample BMW-33 (46, 023/b); sample BMW-35 (47, 022/b). (48) Crococapsa sp. aff. C. truncata (Wu), sample BMW-28 (48, 057/b). (49-52) Crococapsa sp. A, sample BMW-13c (49, 009/b; 50, 073/b; 51, 007/b; 52, 004/b). (53) Belleza decora (Rüst), sample BMW-13c (53, 012/b). 


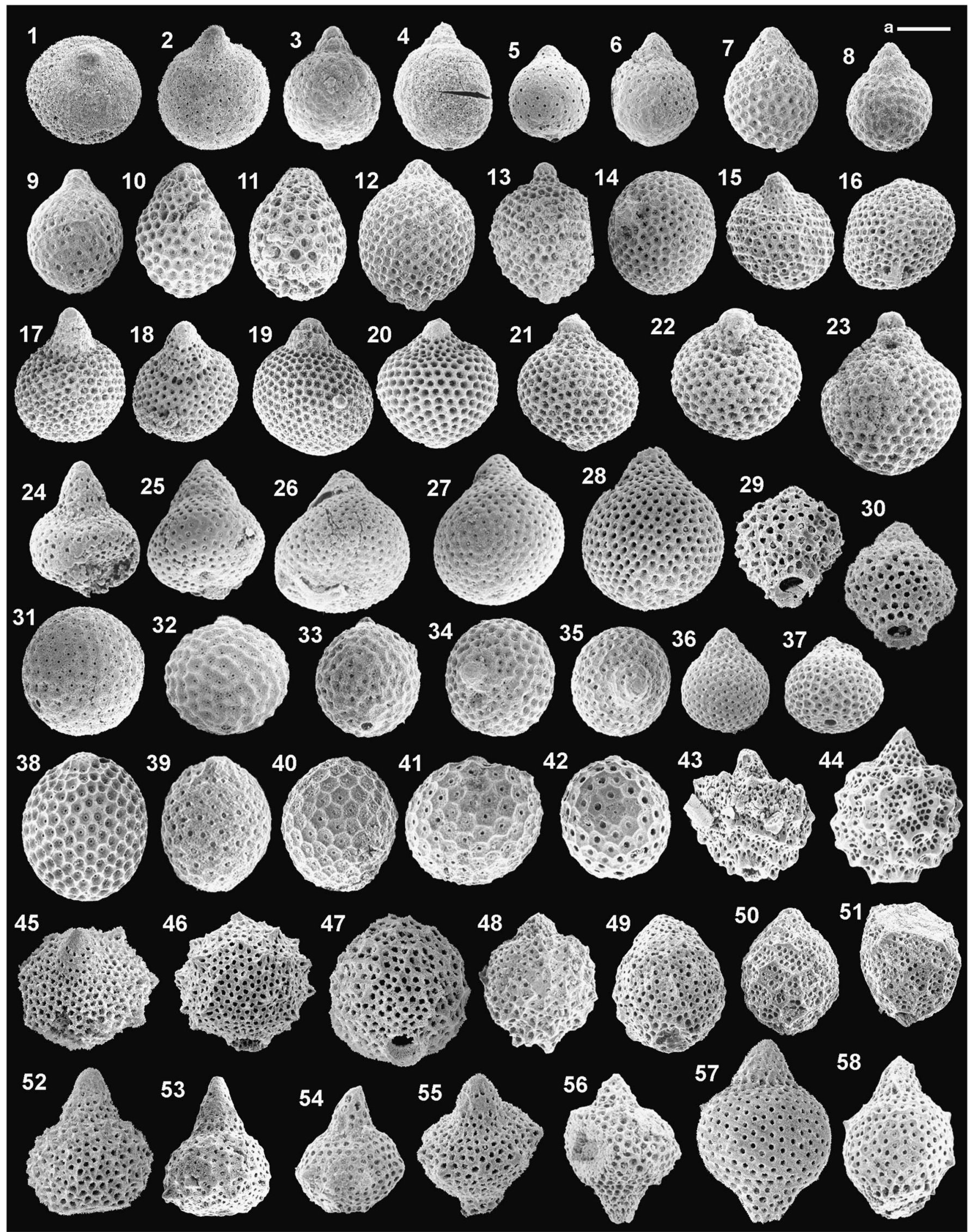


Diagnosis.-Shell with four segments (rarely more), generally thick-walled. Cephalis small, spherical, poreless, without apical horn. Other segments latticed with polygonal pore frames and lacking tubercles or nodes. Last segment somewhat inflated and having an aperture.

Etymology.-From dolium (barrel) and capsa (box); feminine gender.

Occurrence.-Lower Toarcian to Tithonian.

Remarks.-This new genus is differentiated from Praewilliriedellum Kozur, 1984, by having very distinct strictures and well-differentiated segments.

Doliocapsa matsuokai (Yeh, 2009)

Figure 8.33-8.34

1998 Stichocapsa sp. F Arakawa, p. 63, pl. 6, fig. 274.

2005 Stichocapsa sp. Šmuc and Goričan, pl. 3, figs. 22a-b.

2009 Hiscocapsa matsuokai Yeh, p. 67, pl. 21, figs. 1, 8, 20, 22.

2011 Hiscocapsa matsuokai; Yeh, p. 16, pl. 7, figs. 10-13.

2011 Hiscocapsa cf. matsuokai; Yeh, p. 16, pl. 5-7.

Remarks.-The proximal part in our specimens is slightly longer than in the type material. This species differs from all others representatives herein illustrated by having smaller and more numerous polygonal pores, less marked strictures at joints, and longer proximal conical part.

\section{Doliocapsa keni (Kocher, 1981)}

Figure 7.4

1981 Solenotryma keni Kocher, p. 91, pl. 16, figs. 11-12.

2006 Stichomitra (?) keni (Kocher); O’Dogherty et al., p. 442, pl. 5, fig. 6.

Doliocapsa planata $(\mathrm{Wu}, 2000)$

Figure 8.35-8.37

2000 Hiscocapsa planata Wu, p. 303, pl. 1, figs 7-8.
2002 Stichocapsa magnipora Chiari, Marcucci and Prela, p. 76, pl. 3, figs. 13-17.

Genus Hiscocapsa O’Dogherty, 1994

Type species.-Cyrtocapsa grutterinki Tan, 1927.

Other species.-Sethocapsa aitai Chiari, Marcucci, and Prela, 2002; Sethocapsa kodrai Chiari, Marcucci, and Prela, 2002; Stichocapsa pseudornata Tan, 1927; and Stichocapsa rutteni Tan, 1927.

Emended diagnosis.-Hiscocapsa is emended in order to include only tetracyrtid nassellarians having a globose postabdominal segment with tubercles. A large distal aperture is also presented and sometimes, when preserved, an appendage. We note that terminally closed species with tubercles (e.g., Sethocapsa kaminogoensis Aita in Aita and Okada, 1986; Sethocapsa spp., pl. 5, fig. 15 in Aita and Okada, 1986; Sethocapsa sp. 8, pl. 2, fig. 32 in Matsuoka, 1998) should belong to a new genus, which is not described herein.

Occurrence.-Upper Bathonian to upper Aptian.

Hiscocapsa kodrai (Chiari, Marcucci, and Prela, 2002) Figure 9.53

2002 Sethocapsa kodrai Chiari, Marcucci and Prela, p. 74, pl. 3, figs. 1-7.

2003 Quarticella ovalis Takemura; Suzuki and Gawlick, p. 199, fig. 5.40 .

2005 non Sethocapsa kodrai; Nakae and Komuro, fig. 4.47.

2012 Hiscocapsa kodrai; Djeric et al., pl. 1, fig. 23.

Remarks.-This species is very similar to Quarticella ovalis Takemura, but its aperture is much narrower. The larger postabdominal segment also displays well-differentiated tubercles instead of the typical spiny surface of Quarticella ovalis. Hiscocapsa kodrai might belong to Quarticella, but study of the internal structure is required before this new generic assignation is proposed. In the type material, a flattened base with a constricted aperture is clearly distinct (see Chiari et al., 2002, pl. 3, fig. 3). This flattened base is never present in representatives of Quarticella.

Figure 9. Scale bar measures $50 \mu \mathrm{m}$; each radiolarian scanning micrograph is designated by sample and specimen number. (1-6) Hemicryptocapsa buekkensis (Kozur), sample BMW-28 (1, 029; 2, 028); sample BMW-26 (3, 033); sample BMW-31 (4, 032); sample BMW-32 (5, 018); sample BMW-30 (6, 010). (7-8) Zhamoidellum sp. A, sample BMW-32 (7, 051); sample BMW-33 (8, 031). (9) Kilinora? oblongula (Kocher in Baumgartner et al.), sample BMW-32 (9, 005). (10-11) Zhamoidellum sp. aff. Z. ovum Dumitrica, sample BMW-30 (10, 015); sample BMW-32 (11, 086). (12-16) Hemicryptocapsa marcucciae (Cortese), sample BMW-32 (12, 087); sample BMW-26 (13, 074); sample BMW-13c (14, 141); sample BMW-13b (15, 065); sample BMW-30 (16, 043). (17-18) Crococapsa sp. aff. C. tansinhoki (Hull), sample BMW-13c (17, 140; 18, 011). (19) Hemicryptocapsa nonaginta n. sp., BMW-13b (19, 039, paratype). (20-23) Hemicryptocapsa carpathica (Dumitrica), sample BMW-13c (20, 100; 21, 123; 22, 119; 23, 091). (24) Quarkus madstonensis Pessagno, Blome, and Hull in Pessagno et al., sample BMW-31 (24, 029). (25-26) Quarkus japonicus (Yao), sample BMW-13b (25, 026); sample BMW-31 (26, 067), (27-28) Praewilliriedellum convexum (Yao), sample BMW-13b (27, 027); sample BMW-28 (28, 038). (29-30) Quarticella sp. B, sample BMW-28 (29, 056; 30, 010). (31-37) Hemicryptocapsa yaoi (Kozur), sample BMW-13c (31, 116; 32, 112; 34, 118); sample EW-158 (33, 028); sample BMW-33 (35, 011); sample BMW-13b (36, 073); sample BMW-32 (37, 061). (38) Gongylothorax sp. A, sample BMW-34 (38, 019). (39-42), Gongylothorax favosus Dumitrica, sample BMW-32 (39, 058); sample BMW-30 (40, 045); sample BMW-34 (41, 025); sample BMW-33 (42, 036). (43-44) Williriedellum yahazuense (Aita), sample BMW-34 (43, 032); sample BMW-33 (44, 009). (45-48) Williriedellum sp. cf. W. formosum (Chiari, Marcucci, and Prela), sample BMW-28 (45, 049; 46, 033; 47, 058); sample BMW-13b (48, 067). (49) Williriedellum sp. B, sample BMW-13b (49, 053). (50-51) Williriedellum crystallinum Dumitrica, sample BMW-32 (50, 032); sample BMW-30 (51, 007). (52) Quarticella ovalis Takemura, sample BMW-28 (52, 034). (53) Hiscocapsa kodrai (Chiari, Marcucci, and Prela), sample BMW-13b (53, 030). (54-55) Quarticella sp. C, sample BMW-13b (54, 041); sample BMW-28 (55, 050). (56), Quarticella cicciona (Chiari, Marcucci, and Prela), sample BMW-13b (56, 033). (57) Quarticella sp. D, sample BMW-28 (57, 041). (58) Quarticella sp. A, sample EW-158 (58, 065). 


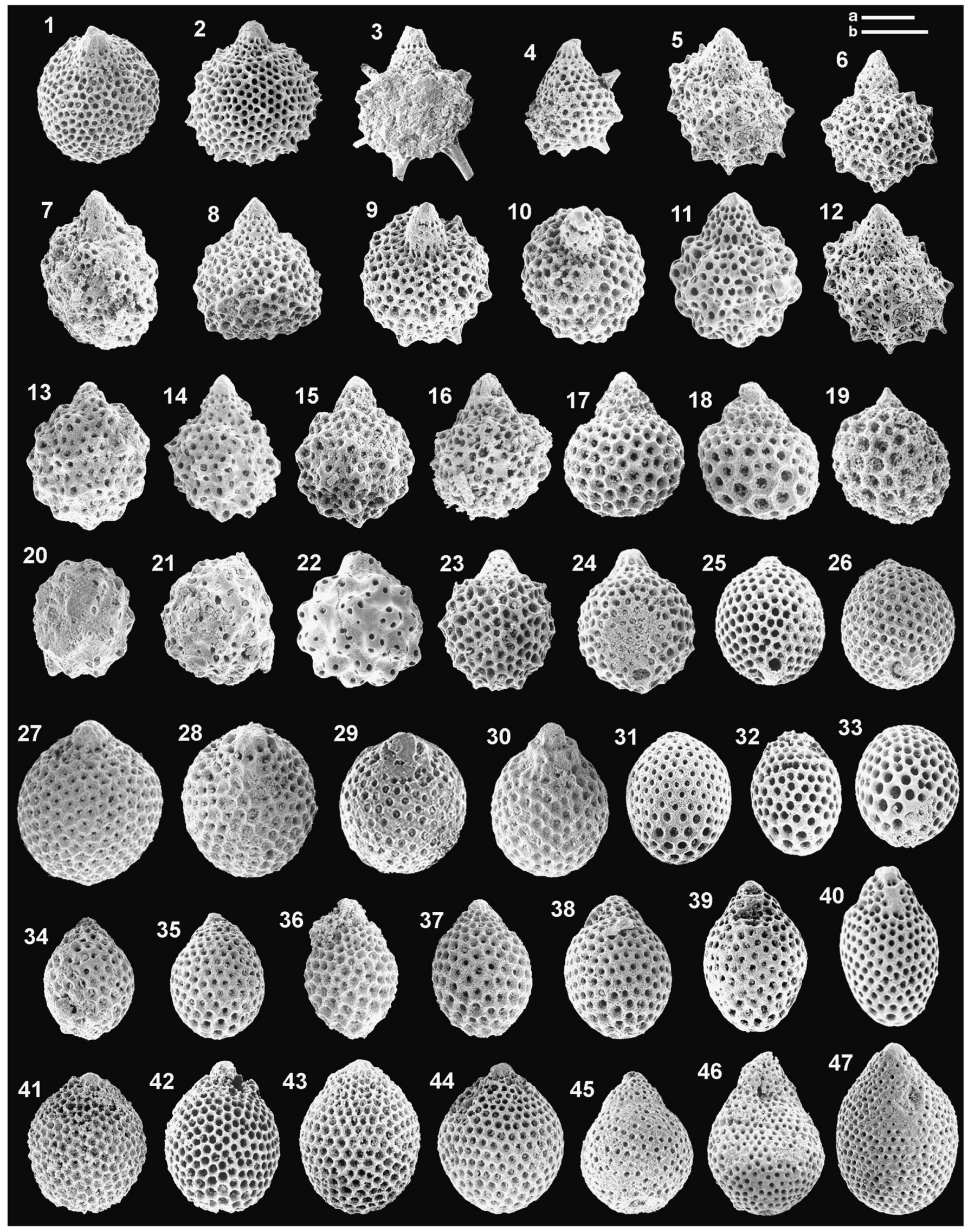


Genus Praewilliriedellum Kozur, 1984

Type species.—Praewilliriedellum cephalospinosum Kozur, 1984.

Other species.-Stichocapsa? pseudoconvexa Kemkin and Taketani, 2004; Stichocapsa convexa Yao, 1979; Stichocapsa robusta Matsuoka, 1984; and Theocorys renzae Schaaf, 1981.

Emended diagnosis.-Praewilliriedellum is emended in order to allocate clear tetracyrtid forms (e.g., Stichocapsa robusta Matsuoka). Many representatives of this genus have an abdomen partially encased in the postabdominal segment, making it hard to differentiate externally. The degree of encasement is nevertheless much lower than in Williriedellum and other genera assigned to Williriedellidae.

Occurrence.-Upper Aalenian to upper Barremian.

\section{Praewilliriedellum convexum (Yao, 1979)}

Figure 9.27-9.28

1979 Stichocapsa convexa Yao, p. 35, pl. 5, figs. 14-16; pl. 6, figs. 1-7.

2003 Stichocapsa convexa; Suzuki and Gawlick, p. 212, fig. 6.51 .

2006 Stichocapsa convexa; O’Dogherty et al., p. 441, pl. 6, fig. 35. [See for complete synonymy]

2007b Stichocapsa convexa; Gawlick et al., figs. 7.15, 8.32, 17.23, 18.10.

2008 Praewilliriedellum convexum; Beccaro et al., pl. 2, fig. 26.

2009 Stichocapsa convexa; Suzuki and Gawlick, p. 186, figs. 5.54A-B.

2013 Praewilliriedellum convexum; Chiari et al., fig. 13n.

Praewilliriedellum robustum (Matsuoka, 1984)

Figure 10.45?, 10.46-10.47

1984 Stichocapsa robusta Matsuoka, p. 146, pl. 1, figs. 6-13; pl. 2, figs. 7-12.

2003 Stichocapsa robusta; Suzuki and Gawlick, p. 213, fig. 5.44.

2006 Stichocapsa robusta; O’Dogherty et al., p. 441, pl. 6, figs. 31-34. [See for complete synonymy]

2007b Stichocapsa robusta; Gawlick et al., fig. 8.34.

2008 Praewilliriedellum robustum; Beccaro et al., pl. 2, fig. 27.

2013 Praewilliriedellum robustum; Chiari et al., fig. 13p.
Genus Quarkus Pessagno, Blome, and Hull in Pessagno et al., 1993

Type species.-Quarkus madstonensis Pessagno, Blome, and Hull in Pessagno et al., 1993.

Other species.—Stichocapsa japonica Yao, 1979.

Occurrence.-Lower Bajocian to upper Callovian. A very similar specimen, but questionable to this genus, was found in upper Berriasian material of the Mariana Trench (Stichocapsa sp. 8 Matsuoka, 1998, pl. 2, fig. 24). We do not assign this species to Quarkus because continuous record from the Callovian to the Berriasian has not been demostrated yet.

Quarkus japonicus (Yao, 1979)

Figure 9.25-9.26

1979 Stichocapsa japonica Yao, p. 36, pl. 6, figs. 8-12; pl. 7, figs. 1-15.

2006 Stichocapsa japonica; O’Dogherty et al., p. 441, pl. 6, fig. 6. [See for complete synonymy]

2009 Stichocapsa japonica; Suzuki and Gawlick, p. 186, figs. 5.53.

2012 Praewilliriedellum japonicum; Goričan et al., pl. 1, fig. 20.

2013 Praewilliriedellum japonicum; Chiari et al., fig. 13o.

Remarks.-This species has always been assigned to the genus Stichocapsa (considered as a nomen dubium by O'Dogherty et al., 2009, p. 330), however we note that this commonly illustrated species is very close to Quarkus madstonensis, the only difference being the less-expanded fourth segment. Judging from the stratigraphic distribution, Quarkus japonicus may be the ancestor of Quarkus madstonensis. An intermediate form (Stichocapsa sp. aff. S. japonica Yao) was illustrated by Chiari et al., 2002 (pl. 3, figs. 18-22) from the upper Bajocian-lower Bathonian of Albania.

Quarkus madstonensis Pessagno, Blome, and Hull in Pessagno et al., 1993

Figure 9.24

1993 Quarkus madstonensis Pessagno, Blome, and Hull in Pessagno et al., p. 159, pl. 8, figs. 9, 13-15, 24.

2006 Williriedellum madstonense; O'Dogherty et al., p. 446, pl. 6, figs. 9-10.

2008 Williriedellum madstonense; Danelian et al., pl. 1, fig. 11.

Figure 10. Scale bars (a, b) measure $50 \mu \mathrm{m}$; each radiolarian scanning micrograph is designated by sample and specimen number, followed by the corresponding scale. (1-2) Williriedellum? sp. C, sample BMW-13b (1, 066/a); sample BMW-28 (2, 007/a). (3) Arcanicapsa exquisita (Hull), sample BMW-32 (3, 013/a). (4) Arcanicapsa sp. A, sample BMW-13b (4, 029/a). (5-16) Arcanicapsa funatoensis (Aita), sample BMW-26 (5, 035/a; 6, 031/a; 10, 037/a; 13, 035/a; 15, 032/a); sample EW-158 (7, 021/a; 8, 029/a; 9, 019/a); sample BMW-34 (11, 011/a); sample BMW-13b (12, 103/a); sample BMW-13c (14, 106/a; 16, 132/a). (17-19) Zhamoidellum ventricosum Dumitrica, sample BMW-13c (17, 121/a; 18, 114/a; 19, 136/a). (20-22) Arcanicapsa undulata (Heitzer), sample BMW-32 (20, 024/a); sample BMW-33 (21, 032/a); sample BMW-34 (22, 016/a). (23-24) Williriedellum sp. A, sample BMW-26 (23, 059/a); sample BMW-13b (24, 074/b). (25-26) Kilinora sp. aff. K. oblongula (Kocher in Baumgartner et al.), sample EW-158 (25, 027/b); sample BMW-32 (26, 047/b). (27-30) Zhamoidellum sp. B, sample BMW-13c (27, 093/b); sample BMW-35 (28, 033/b); sample BMW-32 (29, 084/b); sample BMW-30 (30, 013/b). (31-40) Zhamoidellum ovum Dumitrica, sample BMW-33 (31, 021/a; 32, 012/a; 33, 016/a; 35, 017/a; 39, 034/a); sample BMW-32 (34, 046/a); sample BMW-13c (36, 130/a; 37, 101/a); sample BMW-35 (38, 034/a); sample BMW-34 (40, 018/a). (41-44) Zhamoidellum sp. C, sample BMW-26 (41, 005/a; 44, 063/a); sample BMW-35 (42, 035/a); sample EW-158 (43, 004/a). (45-47) Praewilliriedellum robustum (Matsuoka), sample EW-158 (?45, 025/b); sample BMW-31 (46, 071/a); sample BMW-34 $(47,017 / a)$. 


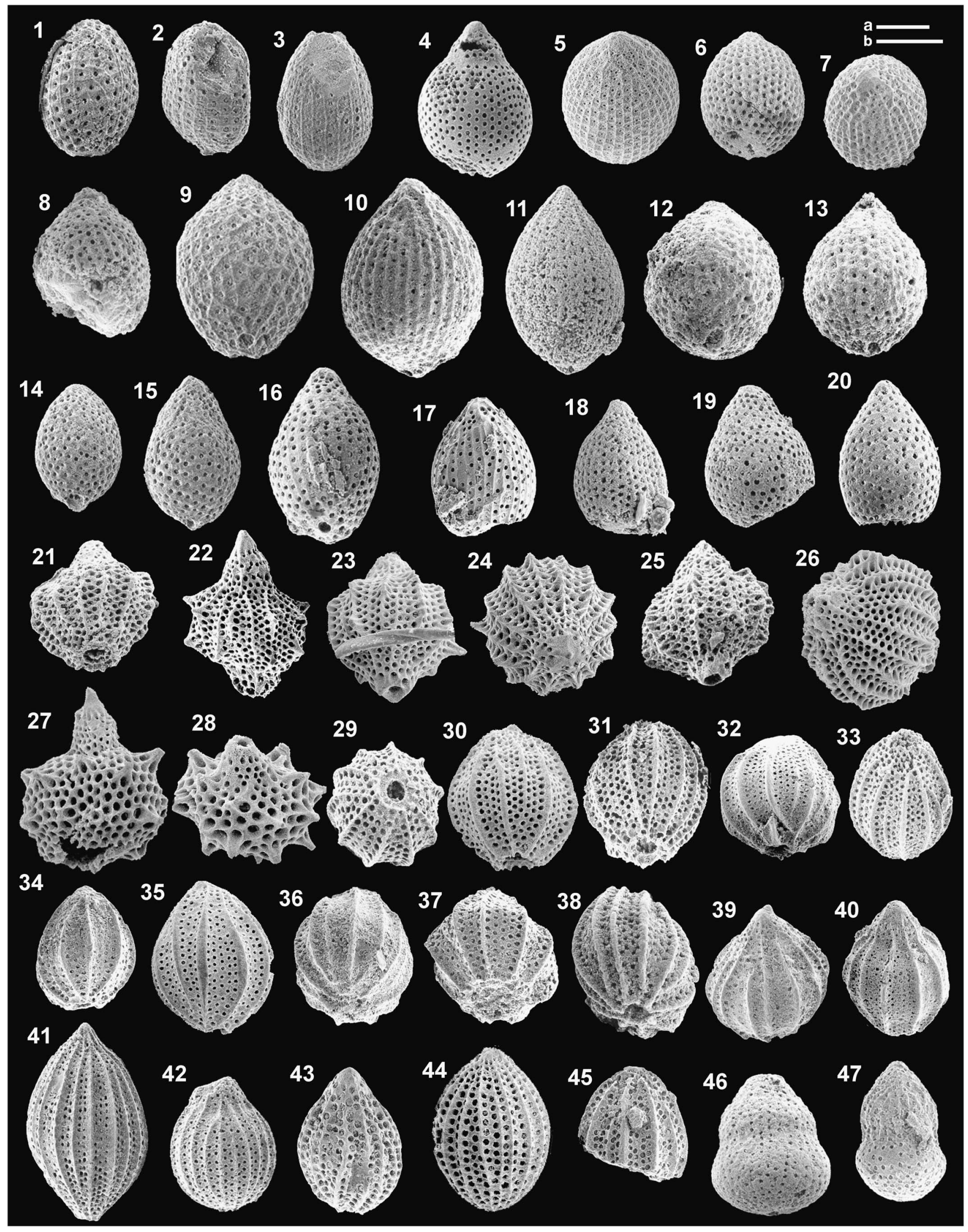


Family Arcanicapsidae Takemura, 1986

Subfamily Arcanicapsinae Takemura, 1986

Included genera.-Arcanicapsa Takemura, 1986; Fultacapsa Ožvoldová in Ožvoldová and Frantová, 1997; Religa Whalen and Carter, 2002; Squinabollum Dumitrica, 1970; Trisyringium Vinassa de Regny, 1901; and the group of species assigned to Dorypyle Squinabol, 1904. Dorypyle should be regarded as a nomen dubium that requires a new genus for taxonomic stability.

The genera Arcanicapsa Takemura, 1986 and Yamatoum Takemura, 1986 were questionably placed under this family in the recent revision by O'Dogherty et al., 2009, but, as stated by Takemura 1986, the cephalic structure has more affinities with the genus Unuma Ichikawa and Yao, 1976. In this paper we follow the same opinion. In the same revision by O'Dogherty et al., 2009, the genus Solidea Whalen and Carter in Carter et al., 1998 was erroneously placed in this subfamily, but its correct placement should be in Favosyringiinae.

Genus Arcanicapsa Takemura, 1986

Type species.—Arcanicapsa sphaerica Takemura, 1986.

Other species.-Sethocapsa aculeata Cortese, 1993; Sethocapsa congduensis $\mathrm{Li}$ and $\mathrm{Wu}, 1985$; Sethocapsa echinata $\mathrm{Li}$ and Wu, 1985; Sethocapsa leiostraca Foreman, 1973b; Sethocapsa trachyostraca Foreman, 1973b; Sethocapsa funatoensis Aita, 1987; and Zhamoidellum? exquisita Hull, 1997.

Occurrence.--Lower Toarcian to lower Aptian.

Remarks.-Arcanicapsa as originally described has a stout apical horn (Takemura 1986, p. 54). In this paper we broaden the definition to include morphologically closely similar species without apical horn. Such tricyrtids, having a closed abdomen ornamented with spines or nodes, have been traditionally ascribed to Sethocapsa (e.g., Sethocapsa funatoensis Aita), which is considered nomen dubium (O'Dogherty et al., 2009). Some species were questionably assigned to Zhamoidellum (e.g., Zhamoidellum? exquisita Hull). Here we assign to Zhamoidellum only species without tubercles (see discussion under Zhamoidellum). Species with a clear distinction between upward-directed spines proximally and downwarddirected spines distally belong to Trisyringium Vinassa de Regny (see O'Dogherty, 1994, p. 208; O'Dogherty et al., 2009).
Arcanicapsa exquisita (Hull, 1997)

Figure 10.3

1997 Zhamoidellum (?) exquisita Hull, p. 132, pl. 38, figs. 5, 16-17, 21.

2000 Zhamoidellum (?) exquisita; Wu, pl. 1, figs. 14-15.

2003 Zhamoidellum exquisita; Suzuki and Gawlick, p. 204, fig. 6.55 .

Arcanicapsa funatoensis (Aita, 1987)

Figure 10.5-10.16

1987 Sethocapsa funatoensis Aita, p. 73, pl. 2, figs. 6a-7b.

2005b Arcanicapsa funatoensis; Nishihara and Yao, fig. 5.1.

2006 Zhamoidellum funatoense; O'Dogherty et al., p. 445, pl. 10, fig. 32. [See for complete synonymy]

2009 non Zhamoidellum funatoense; Yeh, p. 68, pl. 21, figs. 10-11, 17, 25.

2011 Hiscocapsa funatoense; Yeh, p. 16, pl. 7, figs. 19-20, $23,26$.

Arcanicapsa sp. aff. A. funatoensis (Aita, 1987)

Figure 11.27-11.28

Remarks.-The specimens resemble A. funatoensis, but differ by having stronger spines and larger shell size.

Arcanicapsa undulata (Heitzer, 1930)

Figure 10.20-10.22

1930 Lithobotrys undulata Heitzer, p. 390, pl. 28, fig. 22.

2003 Tricolocapsa undulata; Suzuki and Gawlick, figs. 5.41, 6.39.

2006 Tricolocapsa undulata; Auer et al., fig. 6.44.

2007b Tricolocapsa undulata; Gawlick et al., fig. 8.41.

2008 Tricolocapsa undulata; Auer et al., fig. 9.78.

2009 Tricolocapsa undulata; Suzuki and Gawlick, p. 183, figs. 5.44A-B, 5.45A-B, 6.18A-B, 6.19A-B.

Remarks.-Arcanicapsa undulata differs from A. funatoensis by having a smoother surface. Circular pores are present only on tubercles.
Arcanicapsa sp. A

Figure 10.4

Figure 11. Scale bars measure $50 \mu \mathrm{m}$ for a magnification of $\mathrm{x} 200$ (a) and $\mathrm{x} 250$ (b). Each radiolarian scanning micrograph is designated by the sample and the specimen number followed by the corresponding scale. (1-3) Gongylothorax marmoris Kiessling in Kiessling and Zeiss, sample BMW-32 (1, 097/b; 2, 002/b; 3, 057/b). (4) Japonocapsa sp. A, sample BMW-13b (4, 072/b). (5-7) Striatojaponocapsa conexa (Matsuoka), sample EW-158 (5, 003/a; 6, 001/a); sample BMW-32 (7, 089/a). (8-9) Striatojaponocapsa riri O’Dogherty, Goričan, and Dumitrica in O'Dogherty et al., sample BMW-32 (8, 056/b; 9, 008/b). (10-13) Striatojaponocapsa synconexa O’Dogherty, Goričan, and Dumitrica in O'Dogherty et al., sample EW-158 (10, 006/a; 12, 056/b; 13, 071/b); sample BMW-26 (11, 023/b). (14-16) Striatojaponocapsa spp., sample BMW-32 (14, 101/b; 16, 060/b); sample BMW-34 (15, 030/b). (17-20) Striatojaponocapsa? spp., sample BMW-32 (17, 025/a; 19, 059/b); sample BMW-13b (18, 024/b); sample EW-158 (20, 073/b). (21-26) Unuma latusicostatus (Aita), sample BMW-13b (21, 059/a; 25, 069/a); sample EW-158 (22, 023/a); sample BMW-28 (23, 055/a; 24, 005/a; 26, 026/a). (27-28) Arcanicapsa sp. aff. A. funatoensis (Aita), sample BMW-28 (27, 011/a; 28, 031/a). (29-38, 45) Unuma gordus Hull 1997, sample BMW-31 (29, 054/b; 37, 031/a); sample BMW-28 (30, 039/a; 35, 048/a); sample EW-158 (31, 024/a; 36, 058/a); sample BMW-13b (32, 025/a; 34, 055/a); sample BMW-26 (33, 061/a; 38, 072/a); sample BMW-32 (45, 066/a). (39-40) Protunuma turbo Matsuoka, sample BMW-13b (39, 058/a; 40, 052/a). (41) Protunuma japonicus Matsuoka and Yao, sample BMW-33 (41, 014/a). (42) Protunuma europeus O’Dogherty, Goričan and Dumitrica in O'Dogherty et al., sample BMW-13b (42, 061/b). (?43-44) Protunuma ochiensis Matsuoka, sample BMW-33 (43, 013/a); sample BMW-28 (44, 052/a). (46), Theocapsomella cordis (Kocher in Baumgartner et al.), sample BMW-13b (46, 020/b). (47) Theocapsomella medvednicensis (Goričan in Halamić et al.), sample BMW-32 (47, 003/b). 


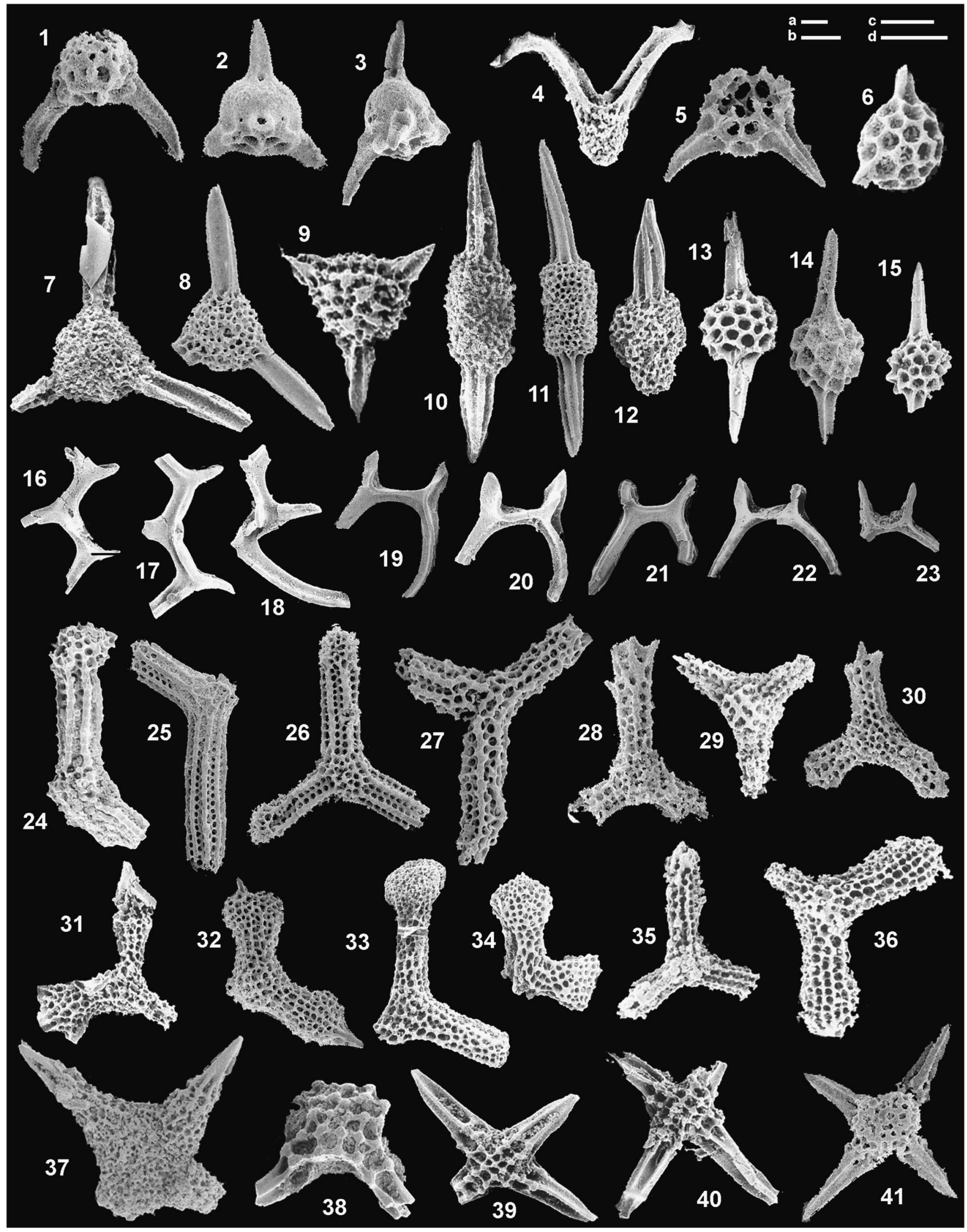


Remarks.-This specimen is similar to Arcanicapsa exquisita but possesses a smaller abdomen; the constriction between thorax and abdomen (lumbar stricture) is less pronounced.

Subfamily Favosyringiinae Steiger, 1992

Genus Spinosicapsa Ožvoldová, 1975

Type species.—Spinosicapsa ceblienica Ožvoldová, 1975.

Occurrence.-Upper Carnian to lower Aptian.

Spinosicapsa basilica (Hull, 1997)

Figure 8.28

1997 Podobursa basilica Hull, p. 100, pl. 41, figs. 7-8, 10, $18,20-21$.

Spinosicapsa lata (Yang, 1993)

Figure 8.30

1993 Syringocapsalata lata Yang, p. 132, pl. 24, figs. 1-2, 16, 20-21; pl. 26, figs. 7, 11, 15.

1997 Podobursa lata; Hull, p. 102, pl. 42, fig. 6.

2007b Syringocapsa lata; Gawlick et al., fig. 19.37.

Spinosicapsa spinosa (Ožvoldová, 1975)

Figure 8.31

1975 Heitzeria spinosa Ožvoldová, p. 78, pl. 101, fig. 2.

1995b Podobursa spinosa; Baumgartner et al., p. 426, pl. 3230, figs. 1-4. [See for complete synonymy]

Spinosicapsa sp. cf. S. triacantha (Fischli, 1916)

Figure 8.29

2006 Podobursa triacantha; O'Dogherty et al., p. 437, pl. 7, fig. 4. [See for complete synonymy]

Remarks.-The assignment to $S$. triacantha is queried because the lateral spines are broken off in this specimen.

Family Eucyrtidiellidae Takemura, 1986

Genus Eucyrtidiellum Baumgartner, 1984

Type species.—Eucyrtidium? unumaensis Yao, 1979.
Occurrence.-Lower Pliensbachian to upper Tithonian.

Eucyrtidiellum nodosum Wakita, 1988

Figure 8.21-8.23

1988 Eucyrtidiellum nodosum Wakita, p. 408, pl. 4, fig. 29; pl. 5, fig. 16.

2003 Eucyrtidiellum nodosum; Suzuki and Gawlick, p. 217, figs. 5.20, 6.24-6.25, 6.29 .

2006 Eucyrtidiellum nodosum; O'Dogherty et al., p. 442, pl. 3, fig. 3. [See for complete synonymy]

2007b Eucyrtidiellum nodosum; Gawlick et al., fig. 8.9; fig. 17.8.

Eucyrtidiellum ptyctum (Riedel and Sanfilippo, 1974)

Figure 8.20

1974 Eucyrtidium ptyctum Riedel and Sanfilippo, p. 778, pl. 5, fig. 7; pl. 12, fig. 14, not fig. 15.

2003 Eucyrtidiellum ptyctum; Suzuki and Gawlick, p. 218, figs. 6.26-6.27.

2006 Eucyrtidiellum ptyctum; Gawlick et al., figs. 8.10, 9.7.

2006 Eucyrtidiellum ptyctum; Auer et al., fig. 6.14.

2006 Eucyrtidiellum ptyctum; O'Dogherty et al., p. 442, pl. 3, figs. 1-2. [See for complete synonymy]

2007b Eucyrtidiellum ptyctum; Gawlick et al., fig. 7.3; fig. 19.18 .

2009 Eucyrtidiellum ptyctum; Suzuki and Gawlick, p. 188 , fig. 5.63 .

Eucyrtidiellum pustulatum Baumgartner, 1984 Figure 8.26-8.27

1984 Eucyrtidiellum pustulatum Baumgartner, p. 765, pl. 4, figs. 4-5.

2003 Eucyrtidiellum unumaense pustulatum; Suzuki and Gawlick, p. 217, figs. 5.20, 6.24-6.25, 6.29. [See for complete synonymy]

2007b Eucyrtidiellum unumaense pustulatum; Gawlick et al., figs. 8.13, 17.12.

Eucyrtidiellum pyramis (Aita in Aita and Okada, 1986)

Figure 8.19

Figure 12. Scale bars (a, b, c, d) measure $50 \mu \mathrm{m}$; each radiolarian scanning micrograph is designated by sample and specimen number, followed by the corresponding scale. (1) Saitoum pagei Pessagno, sample BMW-28 (1, 015/c). (2-3) Saitoum trichylum De Wever, sample BMW-28 (2, 004/c); sample BMW-26 (3,051/c). (4) Bernoullius cristatus Baumgartner, sample EW-158 (4, 038/d). (5-6) Gorgansium silviesense Pessagno and Blome, sample BMW-28 $(\mathbf{5}, 009 / \mathrm{c})$; sample BMW-13b (6, 008/c). (7-8) Alievium? longispineum Yang and Wang, sample BMW-26 (7, 040/c); sample BMW-28 (8, 023/b). (9) Alievium? sp. aff. A. crassum (Kiessling), sample BMW-26 (9, 069/d). (10-12) Archaeospongoprunum elegans Wu, sample EW-158 (10, 051/b); sample BMW-28 (11, 035/b); sample BMW-34 (12, 023/b). (13-15) Pantanellium riedeli Pessagno, sample EW-158 (13, 050/b); sample BMW-28 (14, 036/b); sample BMW-31 (15, 001/b). (16-18) Hexasaturnalis tetraspinus (Yao), sample EW-158 (16, 033/a; 17, 039/a; 18, 035/c). (19-20) Hexasaturnalis nakasekoi Dumitrica and Dumitrica-Jud, sample BMW-28 (19, 021/a); sample EW-158 (20, 041/a). (21-22) Hexasaturnalis suboblongus (Yao), sample BMW-28 (21, 019/a); sample BMW-158 (22, 036/a). (23) Hexasaturnalis minor (Baumgartner in Baumgartner et al.), sample BMW-13c (23, 071/a). (24-27) Tritrabs ewingi (Pessagno), sample BMW-32 (24, 080/b); sample BMW-28 (25, 016/b; 26, 008/b; 27, 006/b). (28-30) Angulobracchia spp., sample BMW-28 (28, 002/c; 30, 018/c); sample BMW-26 (29, 057/c). (31) Paronaella pristidentata Baumgartner, sample EW-158 (31, 044/b). (32) Homoeoparonaella elegans (Pessagno), sample BMW-28 (32, 012/b). (33-34) Paronaella kotura Baumgartner, sample EW-158 (33, 042/a); sample BMW-34 (34, 033/a). (35-36), Homoeoparonaella spp., sample BMW-31 (35, 060/b; 36, 073/b). (37) Crucella theokaftensis Baumgartner, sample BMW-13c (37, 061/b). (38) Emiluvia sedecimporata (Rüst), sample BMW-32 (38, 069/b). (39-40) Emiluvia salensis Pessagno, sample BMW-26 (39, 044/b); sample EW-158 (40, 030/b). (41), Emiluvia? sp., sample BMW-28 (41, 024/c). 
1986 Eucyrtidium (?) pyramis Aita in Aita and Okada, p. 109 , pl. 6, figs. $8-13$; pl. 7, figs. 1a-b.

1995b Eucyrtidiellum pyramis; Baumgartner et al., p. 216, pl. 3019, figs. 1-2. [See for complete synonymy]

2010 Eucyrtidiellum pyramis; Robin et al., fig. 4.14.

Eucyrtidiellum unumaense (Yao, 1979)

Figure 8.24-8.25

1979 Eucyrtidium (?) unumaensis Yao, p. 39, pl. 9, figs. 1-11.

2003 Eucyrtidiellum unumaense unumaense; Suzuki and Gawlick, p. 216, fig. 6.28.

2006 Eucyrtidiellum unumaense; O’Dogherty et al., p. 443, pl. 3, figs. 4-6. [See for complete synonymy]

2007b Eucyrtidiellum unumaense unumaense; Gawlick et al., figs. 7.5, 17.11, 19.19 .

2009 Eucyrtidiellum unumaense; Suzuki and Gawlick, p. 188 , fig. 5.62 .

Superfamily Williriedelloidea Dumitrica, 1970

Family Williriedellidae Dumitrica, 1970

Genus Hemicryptocapsa Tan, 1927

Type species.-Hemicryptocapsa capita Tan, 1927.

Other species.-Hemicryptocapsa nonaginta new species; Praezhamoidellum yaoi Kozur, 1984; Praezhamoidellum buekkense Kozur, 1984; Tricolocapsa tetragona Matsuoka, 1983; Williriedellum? marcuccii Cortese, 1993; Williriedellum carpathicum Dumitrica, 1970.

Emended diagnosis.-Cryptothoracic tricyrtids lacking nodose outer surface are included in this genus. The shell surface can be ornamented with regular pore frames as in the type species, or smooth as in Hemicryptocapsa buekkensis. Also see extended discussion under the genus Williriedellum.

Occurrence.-Upper Tithonian to upper Aptian.

\section{Hemicryptocapsa buekkensis (Kozur, 1984)}

Figure 9.1-9.6

1984 Praezhamoidellum buekkenses Kozur, p. 54, pl. 3, figs. 1a-b.

1998 Tricolocapsa? bukkense; Cordey, p. 128, pl. 27, fig. 9.

2006 Williriedellum buekkense; O’Dogherty et al., p. 446, pl. 9, figs. 1-3

2008 Williriedellum buekkense; Auer et al., fig. 9.52.

2009 Praezhamoidellum buekkense; Gawlick et al., figs. 5.50A-B.

2012 Hemicryptocapsa buekkensis; Goričan et al., pl. 1, figs. 26, 34.

2012 Williriedellum buekkense; Djerić et al., pl. 3, fig. 2.
Hemicryptocapsa carpathica (Dumitrica, 1970)

Figure 9.20-9.23

1970 Williriedellum carpathicum Dumitrica, p. 70, pl. 9, figs. 56a-b; 57-59; pl. 10, fig. 61.

1995b Williriedellum carpathicum; Baumgartner et al., p 626, pl. 4055, figs. 1-3.

2003 Williriedellum carpathicum; Suzuki and Gawlick, p. 200, fig. 6.74. [See for complete synonymy]

2008 Williriedellum carpathicum; Beccaro et al., pl. 3, fig. 29.

2012 Hemicryptocapsa carpathica; Goričan et al., pl. 2, fig. 20.

Hemicryptocapsa marcucciae (Cortese, 1993)

Figure 9.12-9.16

1993 Williriedellum (?) marcuccii Cortese, p. 180, pl. 7, figs. 6-7.

2006 Williriedellum marcucciae; O’Dogherty et al., p. 446, pl. 9, figs. 28-36. [See for complete synonymy]

2008 Williriedellum marcucciae; Auer et al., fig. 11.46.

2008 Williriedellum (?) marcucciae; Beccaro et al., pl. 3, fig. 30.

2009 Williriedellum marcucciae; Suzuki and Gawlick, fig. 5.25; fig. 6.28 .

2010 Williriedellum marcucciae; Robin et al., pl. 3, fig. 11.

2014 Hemicryptocapsa marcucciae; Šegvić et al., pl. 1, figs. 28A-B.

Remarks.-Like other species in this paper, the absence of tubercles or nodes on the surface of this species justifies the transfer from Williriedellum to Hemicryptocapsa.

\section{Hemicryptocapsa nonaginta new species} Figure 9.19

1993 Tricolocapsa(?) sp A. Pessagno et al., p. 160, pl. 8, fig. 27.

1995b Tricolocapsa sp. S Baumgartner et al., p. 602, pl. 4057, figs. 1-3.

2004 Tricolocapsa sp. S; Ziabrev et al., fig. 5.25.

2006 Tricolocapsa sp. S; Auer et al., fig. 6.45.

?2008 Tricolocapsa sp. S; Auer et al., fig. 9.79.

2008 Williriedellum sp. cf. W. sp. S Baumgartner et al., pl. 1, fig. 4.

2009 Tricolocapsa sp. S; Kokubo and Matsuoka, figs. 4.4-4.6.

2009 non Tricolocapsa sp. S; Nishihara, pl. 11, fig. 270.

?2009 Tricolocapsa aff. ruesti Tan; Nishihara, pl. 11, fig. 268.

2009 Tricolocapsa sp. S; Suzuki and Gawlick, p. 183, figs. 5.47A-B.

Holotype.-Specimen MA7647 from sample MKS7A (illustrated in Baumgartner et al., 1995b pl. 4057, fig. 3) from Kashibara section (MA9), south of Hichiso town, Japan (see Matsuoka, 1995). 
Diagnosis.—Large cryptothoracic tricyrtid with very well defined lumbar stricture and a large abdomen. Outer surface without nodes and covered by hexagonal pore frames. Distal aperture constricted circular without rim.

Occurrence.-Upper Bajocian to lower Bathonian.

Description.-Cryptothoracic subspherical form composed of three distinct segments. Cephalis and thorax partially incased in the abdomen, with a very well defined lumbar stricture. The thorax is truncate-conical and covered by small pores whereas the abdomen is quite large and spherical. The entire surface of the abdomen is covered by a latticed meshwork of polygonal pore frames and a constricted aperture is visible.

Etymology.-The name Nonaginta means ninety, in honor of the prodigious decade in contemporary history of radiolarian research.

Measurements. - (in micrometers; $\mu \mathrm{m}$ ) maximum width of shell 112-142, mean 118; maximum length 113-133, mean 123, based on four specimens.

Remarks.-Hemicryptocapsa nonaginta $\mathrm{n}$. $\mathrm{sp}$. was published in open nomenclature in the Mesozoic radiolarian atlas (Baumgartner et al., 1995b) and since then it has been illustrated in many publications. This species differs from $H$. yaoi by having larger circular pores in the middle of polygonal areas. It differs from $H$. carpathica by having a simple circular aperture without a rim.

\section{Hemicryptocapsa yaoi (Kozur, 1984)}

Figure 9.31-9.37

1984 Praezhamoidellum yaoi Kozur, p. 53, pl. 3, fig. 3a-b.

2003 Williriedellum dierschei Suzuki and Gawlick; Suzuki and Gawlick, p. 201, fig. 6.73. [premature name]

2004 Williriedellum dierschei Suzuki and Gawlick in Gawlick et al., p. 311, pl. 4, figs. 1-6.

2006 Williriedellum dierschei; Auer et al., fig. 6.52.

2006 Williriedellum yaoi; O’Dogherty et al., p. 446, fig. 9, figures 6-12. [See for complete synonymy]

2007b Williriedellum dierschei; Gawlick et al., fig. 8.45, 17.32.

2009 Williriedellum dierschei; Suzuki and Gawlick, p. 179, figs. 5.27A-B, 5.28, 6.48A-B.

2010 Williriedellum yaoi; Robin et al., pl. 3, figs. 18, 20.

2012 Hemicryptocapsa yaoi; Goričan et al., pl. 1, fig. 25; pl. 2, fig. 12.

Genus Williriedellum Dumitrica, 1970

Type species.—Williriedellum crystallinum Dumitrica, 1970.

Included species.-Cryptamphorella crepida O'Dogherty, 1994; Hemicryptocapsa polyhedra Dumitrica, 1970; Hemicryptocapsa prepolyhedra Dumitrica, 1970; Hemicryptocapsa tuberosa Dumitrica, 1970; Sethocapsa yahazuensis Aita, 1987; Tricolocapsa clivosa Aliev, 1967; Tricolocapsa formosa Chiari,
Marcucci, and Prela, 2002; Williriedellum? gilkeyi Dumitrica, 1970; Williriedellum crystallinum Dumitrica, 1970; Williriedellum nodosum Chiari, Marcucci, and Prela, 2002; Williriedellum peterschmittae Schaaf, 1981; Williriedellum sujkowskii Widz and De Wever, 1993.

Emended diagnosis.-The genus Williriedellum originally contained cryptothoracic tricyrtids with an aperture regardless of the external ornamentation of the shell. We restrict the name Williriedellum to species with raised ridges or nodes on the outer surface of the abdomen. Species with a regular distribution of circular pores (each pore may be bounded by a simple polygonal pore frame) are assigned to Hemicryptocapsa. The same differentiation between these two genera was applied by O'Dogherty et al. (2009). The concept of that publication, however, did not allow for written definitions and comments.

Occurrence.-Upper Aalenian to lower Coniacian.

\section{Williriedellum crystallinum Dumitrica, 1970}

Figure 9.50-9.51

1970 Williriedellum crystallinum Dumitrica, p. 69, pl. 10, figs. $60 \mathrm{a}-\mathrm{c}, 62-63$.

2009 Williriedellum crystallinum; Suzuki and Gawlick, p. 178, fig. 5.24. [See for complete synonymy]

Williriedellum sp. cf. W. formosum (Chiari, Marcucci, and Prela, 2002)

Figure 9.45-9.48

2002 Tricolocapsa formosa Chiari, Marcucci, and Prela, p. 83, pl. 5, figs. 3-8.

2011 Williriedellum formosum; Bandini et al., pl. 5, figs. 27-28.

2012 Williriedellum formosum; Goričan et al., pl. 1, fig. 23.

Remarks.-The external ornamentation of this species is very close to $W$. formosum but has a rounded aperture whereas in the type material the aperture is covered by a dish-like appendage. Moreover, some of our specimens also have groups of four small pores per frame area like occurring in W. gilkeyi.

\section{Williriedellum yahazuense (Aita, 1987) Figure 9.43-9.44}

1987 Sethocapsa yahazuense Aita, p. 73, pl. 2, figs. 8a-9b; pl. 9, figs. 16-17.

1993 Williriedellum sujkowskii Widz and De Wever, p. 88, pl. 1, figs. 7-10.

2005 Williriedellum yahazuense; Šmuc and Goričan, p. 62, pl. 3, fig. 19.

Williriedellum sp. A

Figure 10.23-10.24

Remarks.-These specimens show very small and pointed nodes on the outer surface of the abdomen. 


\section{Williriedellum sp. B}

Figure 9.49

Remarks.-This morphotype has very faintly developed ridges on the surface. These ridges do not build well-defined frame areas characteristic of $W$. crystallinum.

Williriedellum? sp. C

Figure 10.1-10.2

Remarks.-The genus is queried because the presence of the aperture is not confirmed. The ridges on the surface of the illustrated specimens are only very faintly developed compared to other species of this genus.

\section{Genus Zhamoidellum Dumitrica, 1970}

1992 Complexapora Kiessling in Kiessling and Zeiss.

Type species.—Zhamoidellum ventricosum Dumitrica, 1970.

Included species.-Complexapora kozuri Hull, 1997; Complexapora tirolica Kiessling in Kiessling and Zeiss 1992; Zhamoidellum argandi O'Dogherty, Goričan, and Dumitrica in O'Dogherty et al., 2006; Zhamoidellum boehmi Kiessling, 1999; Zhamoidellum calamin O'Dogherty, Goričan, and Dumitrica in O'Dogherty et al., 2006; Zhamoidellum mikamense Aita, 1986; Zhamoidellum ovum Dumitrica, 1970; Zhamoidellum ventricosum Dumitrica, 1970; Zhamoidellum yehae Dumitrica in Goričan et al., 2006.

Remarks.-As stated by O'Dogherty et al., 2006, the presence or absence of a sutural pore in Zhamoidellum can be regarded as character related to the intraspecific variability. Only species without tubercles are included. Species with spines and nodes are placed in Arcanicapsa.

Occurrence.-Lower Pliensbachian? to upper Tithonian.

Zhamoidellum ovum Dumitrica, 1970

Figure 10.31-10.40

1970 Zhamoidellum ovum Dumitrica, p. 79 pl. 9, figs. 52a-b, 53-54.

2009 Zhamoidellum ovum; Suzuki and Gawlick, p. 179, fig. 5.30A-B, 6.33A-B. [See for complete synonymy]

Zhamoidellum sp. aff. Z. ovum Dumitrica, 1970

Figure 9.10-9.11

Remarks.-These specimens are considered only affinis because they display less-pronounced constrictions and more elongated outline of the shell.

Zhamoidellum ventricosum Dumitrica, 1970

Figure 10.17-10.19

1970 Zhamoidellum ventricosum Dumitrica, p. 79, pl. 9, figs. $55 \mathrm{a}-\mathrm{b}$.
2003 Zhamoidellum ventricosum; Suzuki and Gawlick, p. 205, fig. 6.96 .

2006 Zhamoidellum ventricosum; Auer et al., fig. 6.57.

2006 Zhamoidellum ventricosum; O’Dogherty et al., p. 445, pl. 9, figs. 13-25. [See for complete synonymy]

2009 Zhamoidellum ventricosum; Suzuki and Gawlick, p. 179 , fig. 5.29 .

Zhamoidellum sp. A

Figure 9.7-9.8

Remarks. - This morphotype differs from other Zhamoidellum by its smaller size. The cephalis and thorax are imperforate, but the abdomen is covered by small circular pores set in polygonal pore frames. The collar and lumbar strictures are well marked on these specimens.

\section{Zhamoidellum sp. B}

Figure 10.27-10.30

Remarks.-This morphotype differs from Z. ovum by having a more deeply encased thorax. In addition, the collar stricture is very distinct and the thorax is not porous. The illustrated specimens display enough characters to be considered a new species. However, pictures showing the basal aperture are not available and for this reason this morphotype is not described as a new taxon in this paper.

\section{Zhamoidellum sp. C}

Figure 10.41-10.44

Remarks.-These specimens are very close to Zhamoidellum sp. B, but they differ by having a porous thorax and a lessmarked collar stricture. The pores are more widely open and the surrounding ridges are thinner than in Zhamoidellum sp. B.

Family Japonocapsidae Kozur, 1984

Genus Striatojaponocapsa Kozur, 1984

Type species._Tricolocapsa plicarum Yao, 1979.

Occurrence.-Lower Bajocian to upper Callovian.

Striatojaponocapsa conexa (Matsuoka, 1983)

Figure 11.5-11.7

1983 Tricolocapsa conexa Matsuoka, p. 20, pl. 3, figs. 3-7; pl. 7, figs. 11-14.

2003 Tricolocapsa conexa; Suzuki and Gawlick, p. 208, figs. 5.42, 6.43-45.

2006 Striatojaponocapsa conexa; O’Dogherty et al., p. 447, pl. 10, figs. 18-20. [See for complete synonymy]

2007 Striatojaponocapsa conexa; Hatakeda et al., pl. 2, figs. 1-10.

2007 Striatojaponocapsa conexa; Suzuki and Gawlick, p. 182, figs. 5.40, 6.32A-B. 
Striatojaponocapsa riri O'Dogherty, Goričan, and Dumitrica in O'Dogherty et al., 2006

Figure 11.8-11.9

2006 Striatojaponocapsa riri O’Dogherty, Goričan, and Dumitrica in O'Dogherty et al., p. 447, pl. 8, figs. 14-15.

2007 Striatojaponocapsa riri; Hatakeda et al., pl. 2, figs. 11-20.

Striatojaponocapsa synconexa O'Dogherty, Goričan, and Dumitrica in O'Dogherty et al., 2006

Figure 11.10-11.13

2006 Striatojaponocapsa synconexa O'Dogherty, Goričan, and Dumitrica in O'Dogherty et al., p. 447, pl. 10, figs. 9-17. [See for complete synonymy]

2007 Striatojaponocapsa synconexa; Hatakeda et al., pl. 1, figs. 11-20.

2008 Striatojaponocapsa synconexa; Baumgartner et al., pl. 4, fig. 18.

2012 Striatojaponocapsa synconexa; Goričan et al., pl. 1, figs. 15,35 .

2013 Striatojaponocapsa synconexa; Chiari et al., p. 416, fig. 14c.

Striatojaponocapsa spp.

Figure 11.14-11.16

Remarks.-Various elongated forms of Striatojaponcapsa have been found in our samples. The small number of specimens does not allow the description of new species. They differ from other Striatojaponocapsa by the elongated outline and small size.

\section{Striatojaponocapsa? spp. \\ Figure 11.17-11.20}

Remarks.-The genus is queried because the distal part and the classical appendage on these forms are not preserved, but the surface ornamentation fits well with the general pore patterns of Striatojaponocapsa.

Genus Japonocapsa Kozur, 1984

Type species.—Tricolocapsa? fusiformis Yao, 1979.

Occurrence.-Upper Aalenian to upper Bathonian.

$$
\text { Japonocapsa sp. A }
$$

Figure 11.4

Remarks.-This form is close to Japonocapsa fusiformis (Yao, 1979), but the basal dish-like appendage seems broken off in this specimen.

\section{Family Unumidae Kozur, 1984}

Included genera.-Guttacapsa O'Dogherty, 1994; Helvetocapsa O'Dogherty, Goričan, and Dumitrica in O'Dogherty et al., 2006; Protunuma Ichikawa and Yao, 1976; Quarticella Takemura, 1986; Spinunuma Ichikawa and Yao, 1976 (syn. Unuma); Turbocapsula
O'Dogherty, 1994; Unuma Ichikawa and Yao, 1976; and Yamatoum Takemura, 1986.

Remarks.-According to De Wever et al., 2001 (p. 265) and O'Dogherty et al., 2011 (p. 112) this family was placed under the superfamily Archaeodictyomitroidea. However, the family Unumidae as stated by Takemura, 1986 (p. 36) does not share the same cephalic structure. In this paper, we prefer to consider this family as more closely related to the Japonocapsidae. We also tentatively reassign the genera Quarticella and Yamatoum under this family because the cephalic structure (Yamatoum-type) is the same as in Unuma.

Genus Protunuma Ichikawa and Yao, 1976

Type species.—Protunuma fusiformis Ichikawa and Yao, 1976

Occurrence.-Middle Toarcian to upper Tithonian.

Protunuma europeus O'Dogherty, Goričan, and Dumitrica in O'Dogherty et al., 2006

Figure 11.42

2006 Protunuma europeus O'Dogherty, Goričan, and Dumitrica in O'Dogherty et al., p. 433, pl. 10, figs. 6-8.

Protunuma japonicus Matsuoka and Yao, 1985

Figure 11.41

1985 Protunuma japonicus Matsuoka and Yao, p. 130, pl. 1, figs. 11-15; pl. 3, figs. 6-9.

1995b Protunuma japonicus; Baumgartner et al., p. 434, pl. 3292, fig. 1-8.

2003 Protunuma multicostatus (Heitzer); Suzuki and Gawlick, p. 197, fig. 5.43. [See for complete synonymy]

\section{Protunuma ochiensis Matsuoka, 1983}

Figure 11.43?-11.44

1983 Protunuma (?) ochiensis Matsuoka p. 26, pl. 4, figs. $8-11$; pl. 9, figs. 3-7.

2003 Protunuma ochiensis; Suzuki and Gawlick, p. 197, fig. 6.90.

2006 Protunuma ochiensis; O’Dogherty et al., p. 433, pl. 7, figs. 11-13. [See for complete synonymy]

Protunuma turbo Matsuoka, 1983

Figure 11.39-11.40

1983 Protunuma turbo Matsuoka, p. 24, pl. 4, figs. 4-7; pl. 8, figs. 16-18; pl. 9, figs. 1-2.

1995b Protunuma turbo; Baumgartner et al., p. 436, pl. 4034, figs. 1-3. [See for complete synonymy]

2008 Protunuma turbo; Baumgartner et al., pl. 4, figs. 2-3.

2012 Protunuma turbo; Goričan et al., pl. 1, fig. 28.

Genus Unuma Ichikawa and Yao, 1976

1976 Spinunuma Ichikawa and Yao. 
Type species.-Unuma (Unuma) typicus Ichikawa and Yao, 1976.

Occurrence.--Lower Toarcian to upper Bathonian.

Unuma gordus Hull, 1997

Figure 11.29-11.38, ?11.45

1997 Unuma gorda Hull, p. 172, pl. 43, figs. 9, 11-12.

2003 Unuma gorda; Suzuki and Gawlick, p. 98; figs. 5.36, 6.68 .

2006 Unuma gordus; O'Dogherty et al., p. 434, pl. 7, figs. 15-18. [See for complete synonymy]

2007 Unuma gorda; Gawlick et al., fig. 7.21; fig. 8.44; figs. 17.30, 18.13 .

2009 Unuma gordus; Suzuki and Gawlick, p. 177, figs. 6.2A-B.

2013 Unuma gordus; Chiari et al., fig. 14n.

Unuma latusicostatus (Aita, 1987)

Figure 11.21-11.26

1987 Tricolocapsa latusicostata Aita, p. 76, pl. 4, figs. 7a-8b; pl. 10, figs. 8-9.

1995b Unuma latusicostatus; Baumgartner et al., p. 622, pl. 4058, figs. 1-4.

2012 Unuma latusicostatus; Goričan et al., pl. 1, fig. 30.

Genus Quarticella Takemura, 1986

2009 Minutusolla Yeh, p. 72.

Type species.—Quarticella ovalis Takemura, 1986

Other species.—Minutusolla yaoi Yeh, 2009; Quarticella dura Takemura, 1986; Stichocapsa cicciona Chiari, Marcucci, and Prela, 2002.

Emended diagnosis. - We consider under this genus only those species possessing a latticed fourth inflated segment with somewhat spiny surface, but never with long and stout spines.

Occurrence.-Upper Aalenian-upper Bathonian.

Quarticella cicciona (Chiari, Marcucci, and Prela, 2002) Figure 9.56

2002 Stichocapsa cicciona Chiari, Marcucci, and Prela, p. 76, pl. 3, figs. 8-12.

2006 Stichocapsa cicciona; O’Dogherty et al., p. 441, pl. 6, fig. 36 .

\section{Quarticella ovalis Takemura, 1986}

Figure 9.52

1986 Quarticella ovalis Takemura, p. 58, pl. 8, figs. 17-21.

1995b Quarticella ovalis; Baumgartner et al., p. 466, pl. 4078, figs. 1-3.
2003

Quarticella ovalis; Suzuki and Gawlick, p. 199, fig. 5.40.

2005a Quarticella ovalis; Nishihara and Yao, fig. 2.24.

2008 Quarticella ovalis; Auer et al., fig. 9.57.

2009 Quarticella ovalis; Suzuki and Gawlick, figs. 5.23 A-B.

\section{Quarticella sp. A}

Figure 9.58

Remarks.-This specimen differs from $Q$. ovalis by having a smooth surface and more regular arrangement of pores. Circular pores are surrounded by polygonal pore frames. Very small pyramidal spines occur at vertices.

Quarticella sp. B

Figure 9.29-9.30

?1985 Stichocapsa sp. B Yamamoto et al., pl. 7, fig. 6.

Remarks.-These specimens differ from $Q$. ovalis by having meshwork of larger and equally sized pores. Distal segment has a wide aperture with characteristic flat rim.

\section{Quarticella sp. C}

Figure 9.54-9.55

1997 Sethocapsa? sp. D Yao, fig. 542.

1982 Stichocapsa (?) sp. $\alpha$ Mizutani and Koike, pl. 2, figs. 1, 2a-b.

1982 Stichocapsa (?) sp. A Wakita, pl. 3, fig. 8.

Remarks.-This species is very similar to Quarticella sp. D, but bears a row of tubercles in the equatorial part of the inflated postabdominal segment.

\section{Quarticella sp. D \\ Figure 9.57}

Remarks.-This species differs from $Q$. ovalis by having a smooth surface without spines and regular arrangement of circular pores.

Genus Yamatoum Takemura, 1986

Type species._Yamatoum elegans Takemura, 1986.

Other species.—Quarticella conica Takemura, 1986; Quarticella levis Takemura, 1986; Quarticella? quinaria Takemura, 1986; Quarticella spinosa Takemura, 1986; Yamatoum atlanticum O'Dogherty, Goričan, and Dumitrica in O'Dogherty et al., 2006; Yamatoum caudatum Takemura, 1986; Yamatoum connicinum Takemura, 1986; Yamatoum komamiensis Takemura, 1986; Yamatoum spinosum Takemura, 1986.

Emended diagnosis.-Yamatoum is emended in order to include spindle-shaped forms with a large abdomen armed with numerous (three or more) radiate equatorial spines and, when preserved, a strong (frequently tapered to branched) terminal spines. The last inverted conical 
segment may be missing in many specimens due to its delicate 2009 nature.

Occurrence.-Upper Aalenian to upper Bathonian.

Yamatoum spinosum (Takemura, 1986)

Figure 8.38-8.42

1986 Quarticella spinosa Takemura, p. 59, pl. 9, figs. 1-6.

Remarks.-This species has been transferred into Yamatoum because it bears strong lateral spines on its abdomen. Our specimens have shorter spines than the type-material.

Yamatoum sp. A

Figure 8.32

Remarks.-This species differs from $Y$. spinosum by having a narrower final segment and shorter spines.

Superfamily Archaeodictyomitroidea Pessagno, 1976

Family Hsuuidae Pessagno and Whalen, 1982

Genus Hsuum Pessagno, 1977a

1992 Ogivus El Kadiri.

Type species.-Hsuum cuestaense Pessagno, 1977a.

Occurrence.--Lower Pliensbachian to lower Cenomanian.

Hsuum arabicum Dumitrica in Dumitrica et al., 1997 Figure 7.54

1997 Hsuum arabicum Dumitrica in Dumitrica et al., p. 46, pl. 10, figs. 2-3.

1999 Hsuum arabicum; Hori, p. 82, fig. 7.14.

Hsuum obispoense Pessagno, 1977a

Figure 7.55

1977a Hsuum obispoensis Pessagno, p. 82, pl. 8, figs. 3-4.

1977b Hsuum obispoensis; Pessagno, p. 44, pl. 6, fig. 7.

Genus Transhsuum Takemura, 1986

Type species._-Transhsuum medium Takemura, 1986

Occurrence.—Lower Pliensbachian to upper Kimmeridgian.

Transhsuum brevicostatum (Ožvoldová, 1975)

Figure 8.1-8.3

1975 Lithostrobus brevicostatus Ožvoldová, p. 84, pl. 102, fig. 1.

1995b Transhsuum brevicostatus gr.; Baumgartner et al., p. 578, pl. 3181, figs. $1-5$.
Hsuum brevicostatus gr.; Suzuki and Gawlick, p. 168, fig. 5.6. [See for complete synonymy]

Transhsuum maxwelli (Pessagno, 1977a)

Figure 8.4-8.8, 8.10-8.18

1977a Hsuum maxwelli Pessagno, p. 81, pl. 7, figs. 14-16.

2006 Transhsuum maxwelli gr.; O’Dogherty et al., p. 433, pl. 2, figs. 14, 18-23, 25. [See for complete synonymy]

2009 Hsuum maxwelli; Suzuki and Gawlick, p. 168, fig. 5.7. [See for complete synonymy]

Transhsuum sp. E sensu (Yao, 1997) Figure 8.9

1997 Hsuum sp. E sensu Yao, pl. 15, fig. 197.

Remarks.-The main characteristic of this morphotype is its short skeleton consisting of only five segments.

Genus Parahsuum Yao, 1982

Type species._Parahsuum simplum Yao, 1982.

Occurrence.--Lower Hettangian to upper Kimmeridgian.

Parahsuum carpathicum Widz and De Wever, 1993 Figure 7.52-7.53

1993 Parahsuum carpathicum Widz and De Wever, p. 85, pl. 1, figs. 14-16.

1995b Parahsuum sp. S Baumgartner et al., p. 384, pl. 3240, figs. 1-5.

1999 Parahsuum officerense (Pessagno and Whalen); Hori, p. 82 fig. 7.15.

2003 Parahsuum carpathicum; Suzuki and Gawlick, p. 182, fig. 5.30.

2006 Parahsuum carpathicum; O'Dogherty et al., p. 432, pl. 2, figs. 6-13. [See for complete synonymy]

2009 Parahsuum sp. S; Suzuki and Gawlick, p. 167, fig. 5.5.

Parahsuum sp. aff. P. probosum (Pessagno and Whalen, 1982) Figure 7.61

1982 Droltus (?) probosus Pessagno and Whalen, p. 122, pl. 6, figs. 12, 16-17; pl. 12, fig. 12.

Remarks.-This species differs from the typical P. probosum by having a longer shell with broadened last segment.

Parahsuum snowshoense (Pessagno and Whalen, 1982)

Figure 7.50

1982 Lupherium snowshoense Pessagno and Whalen, p. 136, pl. 6, figs. 6, 20; pl. 12, fig. 6.

2006 Parahsuum snowshoense; O'Dogherty et al., p. 432, pl. 1, figs. 1-3.

2007b Parahsuum snowshoense; Gawlick et al., fig. 18.6. 
Remarks.-We also include forms with an apical horn (e.g., Takemura, 1986, pl. 5, fig. 16) in this species.

\section{Parahsuum sp. aff. P. snowshoense (Pessagno and Whalen, 1982) \\ Figure 7.51}

Remarks.-This species differs from $P$. snowshoense by having less-pronounced strictures and partly thickened costae.

\section{Parahsuum sp. 1 sensu O’Dogherty et al., 2006} Figure 7.49

Remarks.-Our specimen is closely related to $P$. snowshoense (Pessagno and Whalen), but differs by having a slender shell not constricted distally. Segments are trapezoidal in outline and possess four longitudinal rows of pores.

Parahsuum sp. 2 sensu O’Dogherty et al., 2006 Figure 7.56-7.60

Remarks.-This species shows an irregular arrangement of costae over most of the shell. The linear arrangement of costae, characteristic of Parahsuum, is only visible in the distalmost part.

Parahsuum sp. 3 sensu O’Dogherty et al., 2006 Figure 7.62-7.63

Remarks.-Shell conical proximally, cylindrical distally. Costae sharp, high in relief, faintly disappearing proximally.

Genus Semihsuum Pessagno, Blome, and Hull in Pessagno et al., 1993

Type species.—Hsuum? inexploratum Blome, 1984.

Occurrence.-Lower Bajocian to upper Callovian. Semihsuum amabile (Aita, 1987)

Figure 7.15-7.16

1985 Archaeodictyomitra (?) amabilis Aita, fig. 6.6.

2006 Hsuum (?) amabile; O’Dogherty et al., p. 431, pl. 1, figs. 21-22. [See for complete synonymy]

2009 Archaeodictyomitra amabilis; Suzuki and Gawlick, p. 169, figs. 5.10, 6.20 A-B.

2012 Semihsuum amabile; Djeric et al., pl. 1, fig. 28.

2013 Archaeodictyomitra (?) amabilis; Chiari et al., Fig. 12b.

Family Archaeodictyomitridae Pessagno, 1976

Genus Belleza Hull, 1997

Type species.—Stichocapsa decora Rüst, 1885.

Occurrence.-Upper Bajocian to upper Callovian.

Belleza decora (Rüst, 1885)

Figure 8.53
1885 Stichocapsa decora Rüst, p. 319, pl. 17, fig. 3.

1990 Sethocapsa (?) lineaplena Yang and Wang, p. 210, pl. 4, figs. 7?, 12-13.

1995b Stichocapsa decora; Baumgartner et al., p. 520 , pl. 3269, figs. 1-2, not figs. 3-4.

1997 Belleza decora (Rüst); Hull, p. 142, pl. 47, figs. 5-6, 14, 22-23

2006 Mictyoditra decora; O’Dogherty et al., p. 431, pl. 1, fig. 23 [See for complete synonymy]

Genus Archaeodictyomitra Pessagno, 1976

Type species.—Archaeodictyomitra squinaboli Pessagno, 1976.

Occurrence.-Lower Pliensbachian to upper Campanian.

Archaeodictyomitra sp. cf. A. annulata Kozur and Mostler in Grill and Kozur, 1986

Figure 7.21

1986 Archaeodictyomitra annulata Kozur and Mostler in Grill and Kozur, p. 257, pl. 10, fig. 2.

Remarks.-As stated by the original authors, this species is very distinct by its hoop-like second-last segment.

Archaeodictyomitra minoensis (Mizutani, 1981)

Figure 7.40-7.42

1981 Pseudodictyomitra minoensis Mizutani, p. 178, pl. 58, fig. 4; pl. 63, figs. 9-10.

1995b Archaeodictyomitra minoensis; Baumgartner et al., p. 104, pl. 3305, figs. 1-5. [See for complete synonymy]

1999 Archaeodictyomitra minoensis; Hori, p. 81, fig. 7.12 .

2008 Archaeodictyomitra minoensis; Auer et al., fig. 9.4

Archaeodictyomitra praeapiarium Cordey, 1998

Figure 7.36-7.37

1982 Archaeodictyomitra apiara (Rüst); Nishizono et al., pl. 3, fig. 4 .

1998 Archaeodictyomitra praeapiarium Cordey, p. 99, pl. 28, figs. 9-10.

Remarks.-According to Cordey 1998, this species differs from A. apiarium by less-pronounced constrictions and continuous costae, also in the most proximal part.

Archaeodictyomitra prisca Kozur and Mostler in Grill and Kozur, 1986

Figure 7.43-7.45

1986 Archaeodictyomitra prisca Kozur and Mostler in Grill and Kozur, p. 258, pl. 8, figs. 3-6; pl. 9, fig. 1.

2006 Archaeodictyomitra prisca; O’Dogherty et al., p. 430, pl. 1, figs. 4-5. [See for complete synonymy] 
Archaeodictyomitra publica (Hull, 1997)

Figure 7.17

1997 Combusta (?) publica Hull, p. 84, pl. 34, figs 3, 13, 20-21.

2006 Combusta (?) publica; O’Dogherty et al., p. 431, pl. 1, fig. 26. [See for complete synonymy]

2011 Parahsuum publicum; Yeh, p. 11, pl. 2, fig. 18.

Archaeodictyomitra rigida Pessagno, 1977a

Figure 7.33-7.35

1977a Archaeodictyomitra rigida Pessagno, p. 81, pl. 7, figs. 10-11.

2003 Archaeodictyomitra rigida; Suzuki and Gawlick, p. 179, fig. 5.18; fig. 6.20 .

2008 Archaeodictyomitra rigida; Auer et al., fig. 9.7.

2009 Archaeodictyomitra rigida; Suzuki and Gawlick, p. 168, fig. 5.9. [See for complete synonymy]

Archaeodictyomitra spelae Chiari, Cortese, and Marcucci in Chiari et al., 1997

Figure 7.48

1997 Archaeodictyomitra spelae Chiari, Cortese, and Marcucci in Chiari et al., p. 64, pl. 1, figs. 13-15.

2006 Archaeodictyomitra spelae; Danelian et al., pl. 1, fig. 11.

Remarks.-This species is distinguished from other Middle Jurassic Archaeodictyomitra species by its long slender shell and the marked strictures between adjacent segments.

Archaeodictyomitra tyaughtonensis Cordey, 1998

Figure 7.38-7.39

Archaeodictyomitra tyaughtonensis Cordey, 1998, p. 98, pl. 28, figs. 5-6.

Remarks.-According to Cordey (1998) this species is characterized by the well-visible segmentation and the fusiform shape.

Archaeodictyomitra sp. aff. A. exigua Blome, 1984

Figure 7.18-7.20

aff. 1984 Archaeodictyomitra exigua Blome, p. 356, pl. 8, figs. 4, 7-8, 10, 12-13; pl. 15, figs. 14-15.

Remarks.-This species resembles Archaeodictyomitra exigua in its conical shape, but differs by lacking the constricted final segment.

Archaeodictyomitra whalenae Kozur and Mostler in Grill and Kozur, 1986

Figure 7.46-7.47

1986 Archaeodictyomitra whalenae Kozur and Mostler in Grill and Kozur, p. 260, pl. 9, figs. 2-5.
2006 Archaeodictyomitra whalenae; O'Dogherty et al., p. 430 , pl. 1, figs. 8-9. [See for complete synonymy]

Genus Thanarla Pessagno, 1977b

Type species.—Phormocyrtis veneta Squinabol, 1903.

Occurrence.-Lower Bathonian to upper Cenomanian.

Thanarla patricki gr. (Kocher, 1981)

Figure 7.22-7.32

1981 Archaeodictyomitra patricki Kocher, p. 57, pl. 12, figs. 14-17.

2003 Archaeodictyomitra patricki; Suzuki and Gawlick, p. 178, fig. 5.19.

2006 Archaeodictyomitra patricki; O'Dogherty et al., p. 430, pl. 1, figs. 15-17. [See for complete synonymy]

2007b Archaeodictyomitra patricki; Gawlick et al., fig. 19.13.

2013 Archaeodictyomitra sp. cf. A. patricki; Chiari et al., Fig. 12c.

Superfamily Amphipyndacoidea Riedel, 1967

Family Amphipyndacidae Riedel, 1967

Genus Eoxitus Kozur, 1985

Type species.—Eoxitus hungaricus Kozur, 1985.

Other species.-Eoxitus baloghi Kozur, 1985 (syn. Eoxitus nodosus); ?Eoxitus brevis Kozur, 1985; Eoxitus elongatus Kozur, 1985; Eoxitus nodosus Kozur, 1985; Parvicingula dhimenaensis Baumgartner, 1984, Triversus kasinzovae Vishnevskaya, 1991; ?Triversus strobilatus Vishnevskaya, 1991;?Triversus triquetrum Vishnevskaya, 1991.

Occurrence.-The stratigraphic occurrence of Eoxitus should be considered as lower Bajocian to Tithonian (see discussion above). The last representative of Eoxitus is E. dhimenaensis, which goes extinct in the Tithonian.

Remarks._Eoxitus is very similar to its allied Tethysetta, but is much more elongated and does not have the characteristic broad spindle shape frequently observed throughout the Cretaceous forms assigned in Tethysetta. By definition, Tethysetta species have well-developed circumferential ridges and do not have spines on tubercles, as is the case of older species of Eoxitus.

The stratigraphic range of both genera in O'Dogherty et al. (2009) was lower Bajocian-lower Aptian. These two genera are clearly distinguishable both morphologically and stratigraphically. At that time, we included the only species bearing faint spines in the Cretaceous-Parvicingula usotanensis Tumanda, 1989. Nonetheless, we currently believe that this species should be assigned to Tethysetta because it displays the characteristic circumferential ridges, although it also possesses somewhat spiny tubercles. This morphotype is stratigraphically disconnected from other Jurassic spiny parvicingulids. 
The genus Tethysetta includes at least the following species: Dictyomitra boesii Parona, 1890; Lithocampe fasciata Rüst, 1898; Parvicingula mashitaensis Mizutani, 1981; Parvicingula usotanensis Tumanda, 1989; Tethysetta cingulifera Dumitrica in Dumitrica et al., 1997; Tethysetta hullae Dumitrica in Dumitrica et al., 1997; Tethysetta ovoidala Dumitrica in Dumitrica et al., 1997; Tethysetta pygmaea Dumitrica in Dumitrica et al., 1997.

\section{Eoxitus baloghi Kozur, 1985}

Figure 6.7-6.11

1985 Eoxitus baloghi Kozur, p. 216, fig. 2c.

1985 Eoxitus nodosus Kozur, p. 218, figs. 2a-b, not fig. 2d.

1997 Parvicingula dhimenaensis ssp. A Baumgartner et al.; Arakawa, pl. 4, fig. 8, not fig. 4 (=E. hungaricus).

1987 Parvicingula sp. B Aita, p. 66, pl. 5, figs. 9a-10b; pl. 11, fig. 1.

1987 Parvicingula sp. C Aita, p. 66, pl. 5, figs. 12a-13b?; pl. 11, fig. 2.

1989 Triversus spinifer Hattori and Sakamoto, pl. 12, fig. D.

2006 Tethysetta baloghi; O'Dogherty et al., p. 436, pl. 3, figs. $20-21$.

\section{Eoxitus? brevis Kozur, 1985}

Figure 7.9

1985 Eoxitus brevis Kozur, p. 217, figs. 2e-f.

Remarks.-This species is questionably assigned to Eoxitus because it has more than three rows of pores per segment.

\section{Eoxitus dhimenaensis (Baumgartner, 1984)}

Figure 6.15-6.16

1976 Amphipyndax sp. Baumgartner and Bernoulli, p. 611, figs. $12 \mathrm{e}, \mathrm{i}, \mathrm{m}$.

1981 Parvicingula boesii (Parona); De Wever and Caby, pl. 2, fig. C.

1981 Parvicingula boesii; Kocher, p. 81, pl. 15, fig. 11 only.

1982 Parvicingula sp. C Aita, pl. 1, figs. 13, 14.

1982 Amphipyndax ? sp. Nishizono et al., pl. 3, fig. 16.

1984 Parvicingula dhimenaensis Baumgartner, p. 778, pl. 7, figs. 2-3, not fig. 4.

1985 Parvicingula dhimenaensis; De Wever and Miconnet, p. 389, pl. 4, figs. 4, 6-8.

1985 Parvicingula dhimenaensis; Yamamoto et al., p. 36, pl. 6, fig. 1.

1986 Parvicingula dhimenaensis; Conti, pl. 1, fig. 1.

1986 Parvicingula dhimenaensis; Kishida and Hisada, fig. 2.4; fig. 8.2.

1986b Parvicingula dhimenaensis; Matsuoka, pl. 2, fig. 12.

1987 Parvicingula dhimenaensis; Aita, p. 66, ?pl. 2, figs. 3a-b, 5a-b; pl. 9, figs. 12-13.
1987 Parvicingula dhimenaensis; Goričan, p. 185, pl. 3, figs. 13-14.

1988 Parvicingula dhimenaensis; Wakita, pl. 4, fig. 10; pl. 5, fig. 7.

1991b Parvicingula dhimenaensis; Matsuoka, pl. 1, fig. 7.

1995b Parvicingula dhimenaensis dhimenaensis; Baumgartner et al., p. 406, pl. 4072, only fig. 1.

1998 Parvicingula dhimenaensis dhimenaensis; Arakawa, pl. 9, fig. 416.

1998 Parvicingula ? sp. B0 Arakawa, pl. 9, fig. 448.

2009 Parvicingula dhimenaensis; Suzuki and Gawlick, p. 169 , figs. $6.9 \mathrm{~A}-\mathrm{B}$.

2012 Eoxitus dhimenaensis; Goričan et al., pl. 1, figs. 12-13.

Eoxitus hungaricus Kozur, 1985

Figure 6.1-6.6

1982 Amphipyndax (?) sp. A Kido et al., pl. 4, figs. 1-2

1982 Parvicingula sp. J Kishida and Sugano, pl. 12, figs. 6-7.

1982 Parvicingula (?) sp. Imoto et al., pl. 3, 6-7.

1985 Eoxitus elongatus Kozur, p. 217, fig. 1h.

1985 Eoxitus hungaricus Kozur, p. 216, figs. 1a-b, d-e.

1985 Parvicingula spinosa Aita, figs. 6.12-6.13.

1992 Eoxitus hungaricus; Ožvoldová, pl. 1, fig. 14; pl. 4, fig. 8.

1994 Parvicingula dhimenaensis Baumgartner; Goričan, p. 80, pl. 24, figs. 12-13.

1995 Parvicingula dhimenaensis; Takahashi and Ishii, pl. 1, fig. 27.

1995b Parvicingula dhimenaensis ssp. A Baumgartner et al., p. 406, pl. 4071, only fig. 1-3.

1997 Parvicingula dhimenaensis; Arakawa, pl. 4, fig. 4, not fig. 8 (=E. baloghi).

1998 Parvicingula dhimenaensis; Arakawa, pl. 9, fig. 417.

2003 Triversus hungaricus; Suzuki and Gawlick, p. 195 , fig. 6.58-6.60.

2009 Triversus hungaricus; Suzuki and Gawlick, p. 170, fig. 5.14; figs. 6.6A-B, 6.7-6.8.

Eoxitus? sp. A

Figure 6.17

1982 Parvicingula decora Pessagno and Whalen; Aubrecht and Ožvoldová, 1994, pl. 4, fig. 1.

1995b Parvicingula(?) spinata; Baumgartner et al., p. 412, pl. 3187, fig. 1 only, fig. 2?

1998 Parvicingula sp. E0 Arakawa, pl. 10, fig. 430.

1998 Parvicingula sp. E1 Arakawa, pl. 10, fig. 431.

Remarks. - The apical horn and pointed nodes enable differentiation from Praeparvicingula Pessagno, Blome, and Hull in Pessagno et al., 1993.

Eoxitus? sp. B

Figure 6.28 
Remarks.-This morphotype is easily distinguished from other species of Eoxitus by is enormous apical horn.

Family Canoptidae Pessagno in Pessagno et al., 1979

Type species.—Canoptum poissoni Pessagno in Pessagno et al., 1979.

Occurrence.-Ladinian to upper Bajocian.

Genus Canoptum Pessagno, 1979

Remarks.-Includes generic concepts of Paracanoptum Yeh, 1987b and Pseudocanoptum Suzuki, 1997.

Canoptum krahsteinense (Suzuki and Gawlick in Gawlick et al., 2004)

Figure 6.33

1985 Canoptum sp. Yamamoto et al., p. 34, pl. 3, fig. 10.

2003 Spongocapsula krahsteinensis Suzuki and Gawlick, p. 189, fig. 6.95. (Premature name)

2004 Spongocapsula krahsteinensis Suzuki and Gawlick in Gawlick et al., p. 313, figs. 4.7-4.10.

2007b Spongocapsula krahsteinensis; Gawlick et al., fig. 7.14.

2009 Spongocapsula krahsteinensis; Ishida, figs. 10.18, 10.35 .

2011 Spongocapsula cf. krahsteinensis; Ishida, fig. 6.23.

2014 Canoptum krahsteinense; Šegvić et al., pl. 1, figs. 8-9.

Genus Cinguloturris Dumitrica in Dumitrica and Mello, 1982

Type species.—Cinguloturris carpatica Dumitrica in Dumitrica and Mello, 1982.

Occurrence.-Upper Bathonian to lower Valanginian.

Cinguloturris carpatica Dumitrica in Dumitrica and Mello, 1982

Figure 6.29-6.30

1982 Cinguloturris carpatica Dumitrica in Dumitrica and Mello, p. 23, pl. 4, figs. 7-11.

2006 Cinguloturris carpatica; O’Dogherty et al., p. 435, pl. 3, figs. 8-9. [See for complete synonymy]

2009 Cinguloturris carpatica; Suzuki and Gawlick, p. 167, figs. 5.2, 6.1A-B.

Cinguloturris getsensis O'Dogherty, Goričan, and Dumitrica in O'Dogherty et al., 2006

Figure 6.31-6.32

2006 Cinguloturris getsensis O'Dogherty, Goričan, and Dumitrica in O'Dogherty et al., p. 435, pl. 3, figs. 10-12. [See for complete synonymy]

Cinguloturris latiannulata (Grill and Kozur, 1986)

Figure 6.34
1986 Canoptum latiannulatum Grill and Kozur, p. 250, pl. 7, figs. 4-5.

Remarks.-This species is closely related morphologically to $C$. carpatica, but differs by having no pores in the intersegmental depressions at strictures.

Family Obeliscoitidae O’Dogherty, 1994

Genus Olanda Hull, 1997

Type species.—Olanda olorina Hull, 1997.

Occurrence.-Lower Bajocian to middle Albian.

Olanda sp.

Figure 7.14

Remarks.-The single specimen found in sample BMW-28 from the lower Bathonian is broken and cannot be determined at species level, but the proximal part is in good agreement with the genus Olanda.

Family Parvicingulidae Pessagno, 1977a

Genus Caneta Pessagno, Blome, and Hull in Pessagno et al., 1993

Type species.—Parvicingula hsui Pessagno, 1977a.

Occurrence.-Lower Kimmeridgian to upper Tithonian.

Caneta hsui (Pessagno, 1977a)

Figure 6.25

1977a Ristola hsui Pessagno, p. 85, pl. 8, figs. 15-16; pl. 9, figs. 1-5.

1984 Ristola hsui; Pessagno et al., p. 29, pl. 4, figs. 2-3.

1993 Parvicingula sp. cf. hsui; Yang, p. 119, pl. 19, figs. 4-5, 14, 17, 21.

1995 Caneta hsui; Hull, p. 16, pl. 1, figs. 6, 10, 18, 22; pl. 6, fig. 10.

1996 Caneta hsui; Kiessling and Scasso, pl. 1, fig. 17.

1999 Caneta hsui s.l.; Kiessling, p. 48, pl. 10, figs. 12-13.

2003 Wrangellium hsuei; Suzuki and Gawlick, p. 194, fig. 6.99.

2007b Wrangellium hsuei; Gawlick et al., fig. 8.48.

Remarks.-Our specimen does not have a constricted last segment as illustrated in the holotype.

Genus Takemuraella new name

1986 Triversus Takemura, preoccupied name (non Triversus Sher 1973, Nematoidea).

Type species.—Triversus japonicus Takemura, 1986. 
Other species._Praeparvicingula tlellensis Carter in Goričan et al., 2006; Triversus fastigatus Hull, 1997; Triversus schardti O'Dogherty, Goričan, and Dumitrica in O'Dogherty et al., 2006; Triversus spinifer Takemura, 1986; Triversus preconicus Vishnevskaya, 1991.

Diagnosis.-Multicyrtid having three rows of circular pores by segment, which are externally weakly defined by ridges or strictures, and usually without apical horn or occasionally a very faint apical horn is present (e.g., Triversus fastigatus Hull 1997, pl. 51, fig. 5).

Occurrence.-Upper Pliensbachian to upper Bathonian.

Etymology.- - In honor of our friend and colleague Prof. Atsushi Takemura, for his great contribution of the taxonomy and stratigraphy of Paleozoic and Mesozoic radiolarians.

Remarks.-Takemura (1986) emphasized the Amphipyndaxtype cephalic skeletal structure of this genus as a distinction from Parvicingula. In addition, we note that Takemuraella shares with other parvicingulids the three rows of pores per segment. but it lacks the typical circumferential ridges always visible in Praeparvicingula and Parvicingula.

Takemuraella japonica (Takemura, 1986)

Figure 6.49-6.50

1986 Triversus japonicus Takemura, p. 62, pl. 16-20.

not Triversus japonicus; Hattori and Sakamoto, pl. 12, 1989 figs. B-C.

2006 Triversus japonicus; O’Dogherty et al., 434, pl. 4, fig. 3.

2013 Triversus japonicus; Chiari et al., p. 418, fig. 14k.

Takemuraella schardti (O'Dogherty, Goričan, and Dumitrica in O’Dogherty et al., 2006)

Figure 6.18-6.21

1997 Ristola (?) turpicula Pessagno and Whalen; Arakawa, pl. 4, fig. 18.

2006 Stichocapsa tuscanica Chiari, Cortese, and Marcucci; Danelian et al., pl. 2, fig. 8.

2006 Triversus schardti O’Dogherty, Goričan, and Dumitrica in O'Dogherty et al., p. 434, pl. 4, figs. 4-10. [See for complete synonymy]

2008 Parvicingula spinata (Vinassa de Regny); Auer et al., fig. 9.47.

2009 Triversus hexagonatus (Heitzer); Suzuki and Gawlick, p. 170 , figs. $5.15 ; 6.11 \mathrm{~A}-\mathrm{B}$.

Takemuraella sp. cf. T. schardti (O’Dogherty, Goričan, and Dumitrica in O'Dogherty et al., 2006)

Figure 7.10-7.11

Remarks.-This species differs from $T$. schardti by having fewer segments, a wider conical shell, and larger pores.

Takemuraella spinifera (Takemura, 1986)

Figure 6.22-6.24
1982 Amphipyndax sp. A Kido et al., pl. 4, figs. 1, 2.

1982 Amphipyndax (?) sp. Kojima, p. 88, pl. 1, fig. 4.

1986 Triversus spinifer Takemura, p. 63, pl. 10, figs. 21-23; pl. 11, figs. 1-2.

1987 Ristola sp. E Hattori, pl. 19, fig. 6.

1987 Triversus aff. T. spinifer Hattori, pl. 20, fig. 7.

1989 Parvicingula (?) sp. Hattori and Sakamoto, pl. 12, fig. A.

1989 Ristola spp. Hattori, pl. 14, fig. H.

1997 Parvicingula aff. spinifer; Yao, pl. 13, fig. 609.

1998 Parvicingula sp. H0 Arakawa, pl. 9, fig. 434.

2003 Parvicingula spinifer; Goričan et al., p. 297, pl. 5, fig. 5.

2004 Triversus spinifer; Matsuoka, fig. 239.

2006 Praeparvicingula? spinifera; Goričan et al., p. 338, pl. TVS01, figs. 1-5.

2012 Eoxitus spinifer (Takemura); Goričan et al., pl. 1, fig. 40.

2013 Triversus sp. cf. T. spinifer Takemura; Chiari et al., fig. 141.

Remarks.-As in Takemuraella schardti, the presence or absence of a delicate and narrower last segment (even broken, see Fig. 6.17-18) is not considered a significant characteristic to separate two morphotypes belonging to different subspecies.

Family Pseudodictyomitridae Pessagno, 1977b

Genus Loopus Yang, 1993

Type species.-Pseudodictyomitra primitiva Matsuoka and Yao, 1985.

Other species.-Candissa mexicana Hull, 1997; Cinguloturris? venusta Chiari, Cortese, and Marcucci in Chiari et al., 1997; Loopus doliolum Dumitrica in Dumitrica et al., 1997; Loopus doliolum martae Beccaro, 2004; Loopus yangi Dumitrica in Dumitrica et al., 1997; Pseudodictyomitra blabla Schaaf, 1981.

Occurrence.-Lower Bathonian to lower Aptian.

Remarks._-Includes generic concepts of Candissa Hull, 1997.

Loopus martae Beccaro, 2004

Figure 7.64-7.66

2004 Loopus doliolum martae Beccaro, p. 13, pl. 1, figs. 3-5. 2008 Loopus doliolum martae; Beccaro et al., pl. 3, fig. 17.

Remarks.-In this paper the record of the taxon is raised to species level. This new taxonomic assignation is justified by the clear morphological distinction and different stratigraphic ranges between Loopus doliolum Dumitrica in Dumitrica et al., 1997 and Loopus martae Beccaro.

Loopus mexicanus (Hull, 1997)

Figure 7.71

1997 Candissa mexicana Hull, p. 144, pl. 47, figs. 11-12, 17, 21, 24. 
Remarks.-Loopus mexicanus is distinguished from other Loopus included by having a lobate outline with wellpronounced constrictions.

Loopus venustus (Chiari, Cortese, and Marcucci in Chiari et al., 1997)

Figure 7.67-7.70

1982? Unnamed multicyrtoid nassellaria Adachi, pl. 2, fig. 7.

1986a Pseudodictyomitra (?) sp. D Matsuoka, pl. 4, only figs. 2-6.

1986b Pseudodictyomitra (?) sp. D Matsuoka, pl. 2, fig. 11; pl. 3, figs. 12a-b.

1988 Pseudodictyomitra sp. D Wakita, pl. 1, fig. 12.

1988 Pseudodictyomitra (?) sp. D Wakita, pl. 4, fig. 12.

1990 Pseudodictyomitra (?) sp. D Matsuoka and Oji, pl. 1, fig. 8.

1997 Cinguloturris (?) venusta Chiari, Cortese, and Marcucci in Chiari et al., p. 66, pl. 2, figs. 4-5.

1997 Cinguloturris (?) sp. Chiari, Cortese, and Marcucci in Chiari et al., pl. 2, fig. 6.

2006 Dictyomitrella (?) sp. 3 O’Dogherty et al., p. 436, pl. 3, fig. 16.

2006 Loopus venustus; Danelian et al., p. S40, pl. 1, figs. 21-23.

Remarks.-We group a large variety of Loopus with complex irregular ornamentation of disconnected short costae and small polygonal depressions in $L$. venustus. This species clearly differs from L. primitivus, which has regularly distributted vertical costae, no polygonal depressions, and stronger strictures.

Genus Mizukidella new genus

Type species.—Dictyomitrella? kamoensis Mizutani and Kido, 1983.

Other species.-Canoptum hungaricum Grill and Kozur, 1986; ?Canoptum rudabanyaense Grill and Kozur, 1986; Mizukidella mokaensis new species.

Diagnosis.-Multicyrtid conical to subcylindrical shell. Cephalis dome-shaped without horn. Abdomen and post-abdominal cylindrical segments separated by nodose circumferential ridges, with paired pores just below and above the ridges. Abdomen and post-abdominal chambers have more or less regularly arranged rows of circular pits.

Etymology.-Achronym of parts of Mizutani, Kido, and Dictyomitrella; feminine gender.

Occurrence.-Bajocian to upper Berriasian.

Remarks.—Mizukidella n. gen. is distinguished from other parvicingulids by having a characteristic surface with pits (very small depressions) on the medial part of post-abdominal segments, which are bounded by circumferential ridges and limited by one row of pores below and above. A possible origin from Canoptum is suspected. Through the latest Jurassic, this genus may have given rise to Svinitzium by the regularization of the size and arrangement of pores and pits around the circumferential ridges.

Mizukidella kamoensis (Mizutani and Kido, 1983)

Figure 6.12-6.14

1983 Dictyomitrella (?) kamoensis Mizutani and Kido, p. 258, pl. 53, figs. 2-4b.

2006 Dictyomitrella (?) kamoensis; O’Dogherty et al., p. 435, pl. 3, fig. 15. [See for complete synonymy]

2009 Wrangellium oregonense Yeh; Yeh, p. 59, pl. 17, figs. 13, 22.

2013 Svinitzium kamoense; Chiari et al., fig. 14d.

Mizukidella mokaensis new species

Figure 6.26-6.27

1998 Praecaneta ? sp. 6 Matsuoka, pl. 9, fig. 129.

Holotype.-The specimen illustrated on figure 6.27 from sample BMW-35, upper Tithonian of Sillenkopf Formation (Northern Calcareous Alps, Austria).

Diagnosis.—Parvicingulid with very small depressions (pits) arranged linearly on the medial part of post-abdominal segments. A middle row of pores is always present in distal segments.

Occurrence.-Middle Oxfordian to upper Berriasian.

Description.-Shell as with genus having commonly a conical shell with eight segments. Cephalis and thorax poreless, conical, and separated by rows of small pores. Distal segments increase gradually in width. Circumferential ridges strong and bearing vertical bars displaying a typical "H-linked" structure. Each segment has two rows of pits at the ridges and a row of small pores in the middle part. Two rows of bigger pores are always present just above and below the ridges. In well-preserved specimens (e.g., Mariana trench, Matsuoka, 1998, pl. 9, fig. 129), a velum on the distalmost segment may be preserved.

Etymology.-Mokaensis is an anagram of kamoensis, which is the other species included into this genus (see above).

Measurements.-(in micrometers; $\mu \mathrm{m}$ ) maximum diameter of conical shell 226-263, mean 240; maximum length 113-119, mean 116, based on three specimens.

Remarks.—Mizukidella mokaensis differs from M. kamoensis by having a more regular shell structure. The pits are linearly arranged, pores are larger, ridges are stronger, and a middle row of pores is always present in distal segments.

Family Xitidae Pessagno, 1977b

Genus Xitus Pessagno, 1977b

Type species.—Xitus plenus Pessagno, 1977b. 
Occurrence.-Upper Bajocian to upper Campanian.

Remarks.-Includes generic concepts of Antexitus Yeh, 2009.

Xitus skenderbegi (Chiari, Marcucci, and Prela, 2002) Figure 7.1-7.3

1993 Xitus? sp. A Cortese, p. 181, pl. 7, figs. 6-7.

1997 Xitus sp. Matsuoka and Baumgartner, pl. 3, fig. 16.

1999 Xitus sp. A Halamić et al., pl. 3, figs. 1-4.

2002 Neorelumbra skenderbegi Chiari, Marcucci, and Prela, p. 68, pl. 1, figs. 14-21.

2003 Neorelumbra skenderbegi; Suzuki and Gawlick, p. 190, fig. 6.32 .

2004 Neorelumbra skenderbegi; Chiari et al., pl. 2, fig. 2.

2005 Xitus skenderbegi; Šmuc and Goričan, p. 62, pl. 4, figs. 15-16.

2008 Xitus skenderbegi; Auer et al., fig. 9.42

2009 Xitus skenderbegi; Suzuki and Gawlick, fig. 5.11.

Xitus sp. A

Figure 7.12-7.13

Remarks.-This species differs from X. skenderbegi by having a more slender shape; it differs from X. magnus Baumgartner in Baumgartner et al., 1995 in being smaller and having fewer segments.

\section{Family Xitomitridae new family}

Type genus.-Xitomitra new genus.

Included genera.-Campanomitra new genus; Parvimitrella new genus; Pseudodictyomitrella Grill and Kozur, 1986; Xitomitra new genus.

Diagnosis.-Multicyrtid nassellarians with small poreless cephalis and all other segments covered by a latticed meshwork of uniform size polygonal pore frames. Shell largely open distally and lacking any kind of distal projections or appendages. The genera are distinguished on the basis of presence or absence of apical horn and presence or absence of stricture at intersegmental constrictions. Only one genus, Xitomitra n. gen., may develop a secondary layer of very faint nodes on distal segments.

Occurrence.-Aalenian to Maastrichtian.

Remarks.-The Xitomitridae $\mathrm{n}$. fam. is related to the Canoptidae, Parvicingulidae, and Xitidae. It differs from the Canoptidae by having a latticed shell with larger pores. It differs from Parvicingulidae by lacking the typical arrangement of horizontal rows of pores per segment. Xitomitridae $\mathrm{n}$. fam. is also distinguished from Xitidae by lacking the typical double layer latticed meshwork bearing large tubercles on the entire surface.

\section{Genus Campanomitra new genus}

Type species.—Stichocapsa praepulchella Hori, 1999.
Other species.-Amphipyndax awaensis Nakaseko and Nishimura in Nakaseko et al., 1979; Amphipyndax conicus Nakaseko and Nishimura, 1981; Amphipyndax ellipticus Nakaseko and Nishimura, 1981; Archicorys pulchella Rüst, 1885; Parvicingula cappa Cortese, 1993; ?Pseudoeucyrtis buekkensis Grill and Kozur, 1986, Quarticella hunzikeri O'Dogherty, Goričan, and Dumitrica in O'Dogherty et al., 2006; ?Stichocapsa labyrinthica Dumitrica in Dumitrica et al., 1997; Stichocapsa tuscanica Chiari, Cortese, and Marcucci in Chiari et al., 1997; Stichocapsa ulivii Chiari, Cortese, and Marcucci in Chiari et al., 1997; Stichocapsa devorata arctica Vishnevskaya and Murchey, 2002; forms included as Stichocapsa spp. by Matsuoka (1998, figs. 35, 37-40).

Diagnosis.-Campanulate shell composed of four to ten segments. Largely open distally. Small, poreless cephalis without apical horn. Other segments covered by a latticed meshwork consisting of relatively large polygonal pore frames. The strictures between junctions of the segments are never present.

Etymology.-Genus name derived from campano (bell) and mitra (ecclesiastical headgear: miter). Feminine gender.

\section{Occurrence.-Bajocian to Cenomanian.}

Remarks.-Campanomitra n. gen. differs from Pseudodictyomitrella by lacking an apical horn. It differs from Parvimitrella and Xitomitra in having larger pores and no intersegmental constrictions. Campanomitra n. gen. differs from Takemuraella by lacking the arrangement of three rows of pores per segment and having no circumferential ridges.

A common character of the genus in Cretaceous species is the presence of a large sutural pore on the surface of the shell. Stichocapsa labyrinthica Dumitrica in Dumitrica et al., 1997 is tentatively assigned in this genus because it lacks the distinctive sutural pore present in all other Cretaceous representatives of the genus.

Campanomitra sp. aff. C. buekkensis (Grill and Kozur, 1986) Figure 6.44

1986 Pseudoeucyrtis buekkensis Grill and Kozur, p. 251, pl. 6, fig. 4.

Remarks.-This specimen is characterized by an irregular arrangement of circular pores throughout the entire shell. It differs from typical C. buekkensis in which the pores are arranged in horizontal rows distally.

Campanomitra tuscanica (Chiari, Cortese, and Marcucci in Chiari et al., 1997)

Figure 6.65-6.69

1986 Pseudodictyomitrella hexagonata; Grill and Kozur, pl. 4, fig. 2.

1997 Stichocapsa tuscanica Chiari, Cortese, and Marcucci in Chiari et al., p. 70, pl. 4, figs. 8-9. 
2003 Triversus hexagonatus; Suzuki and Gawlick, p. 194, figs. 5.48, 6.61.

2006 Pseudodictyomitrella tuscanica; O'Dogherty et al., p. 440, pl. 5, figs. 7-9.

2006 Triversus hexagonatus; Gawlick et al., fig. 8c.40; fig. 9b.20.

2006 Triversus hexagonatus; Auer et al., fig. 6.48.

2007b Triversus hexagonatus; Gawlick et al., fig. 17.29.

2013 Pseudodictyomitrella tuscanica; Chiari et al., p. 415, fig. 13t.

Campanomitra sp. aff. C. tuscanica (Chiari, Cortese, and Marcucci in Chiari et al., 1997)

Figure 6.62-6.64

Remarks.-This species differs from C. tuscanica by having cylindrical instead of conical shell distally.

Campanomitra sp. aff. C. ulivii (Chiari, Cortese, and Marcucci in Chiari et al., 1997)

Figure 6.70-6.73

1997 Stichocapsa ulivii Chiari, Cortese, and Marcucci in Chiari et al., p. 70, pl. 4, figs. 10-11.

?1997 Stichocapsa sp. aff. S. ulivii Chiari, Cortese, and Marcucci in Chiari et al., p. 72, pl. 4, fig. 12.

Remarks.-This species resembles C. ulivii in shape and pore size, but differs by having a smoother surface without raised pore frames.

\section{Campanomitra? sp. A}

Figure 6.40-6.43

Remarks.-The specimens included in this species have a barrel-shape outline (fusiform) and consist of six or more segments.

\section{Campanomitra? spp. \\ Figure 6.56-6.61}

1982 Stichocapsa sp. A Sashida et al., pl. 2, fig. 2.

1989 Bagotum sp. A Hattori and Sakamoto, pl. 13, fig. J.

Remarks.-Various species of Campanomitra n. gen. with fewer than five segments commonly occur in our material. Similar forms have been previously illustrated (see synonymy). However, more in depth taxonomical research is needed to describe new species. This morphotype vaguely resembles the Cretaceous species Trimulus parmatus O'Dogherty, 1994.

\section{Genus Parvimitrella new genus}

Type species.—Pseudodictyomitrella wallacheri Grill and Kozur 1986 (syn. Parvifavus irregularis Takemura, 1986).

Included species.-Amphipyndax plousios Foreman, 1968; Parvifavus irregularis Takemura, 1986; Pseudodictyomitrella wallacheri Grill and Kozur, 1986; Stichomitra? angulata
Bragin and Tekin in Bragin et al., 2002; Stichomitra cathara Foreman, 1968; Stichomitra? cechena Foreman, 1968; Stichomitra communis Squinabol, 1903; Stichomitra compsa Foreman, 1968; Stichomitra magna Squinabol, 1904; ?Stichomitra navalis O’Dogherty, 1994.

Diagnosis.—Thick-walled shell, multisegmented, having a single hexagonal closely packed layer of pores per segment. Strictures between segments usually well marked.

Etymology.-The generic name comes from a combination of Pseudodictyomitrella and Parvifavus, to which the type species was originally assigned.

Occurrence.-Aalenian to Maastrichtian.

Remarks.-Parvimitrella $\mathrm{n}$. gen. is erected to replace the genus Stichomitra Cayeux, 1897, sensu O'Dogherty, 1994, due to problems related to the poor definition of the type species (see discussion regarding this subject in O'Dogherty, 1994; O'Dogherty et al., 2009).

Parvimitrella n. gen. (ex. Stichomitra) is easily distinguished from other Jurassic and Cretaceous multi-segmented forms by its typical pattern of hexagonal close-packed layer of pores per chamber and its frequently dome-shaped cephalis, divided into two chambers by a transverse internal ledge. Parvimitrella n. gen. is distinguished from Amphipyndax in lacking two structurally distinct layers of test material and if circumferential ridges develop, they never occur at segmental divisions.

Parvimitrella n. gen. differs from Pseudodictyomitrella by having a rounded cephalis and strictures at segmental divisions. As mentioned above, many species originally included under the genus Stichomitra (especially in the Cretaceous) belong at least to three distinct described genera: Amphipternis Foreman, 1973a; Schaafella Vishnevskaya in Basov and Vishnevskaya, 1991; and Eostichomitra Empson-Morin, 1981.

Species frequently ascribed to Stichomitra, but now considered as belonging to other genera: Amphipternis: Dictyomitra mediocris Tan, 1927; Stichocapsa? stocki Campbell and Clark, 1944; and Stichomitra tosaensis Nakaseko and Nishimura, 1981.

Schaafella: Schaafella deweveri Vishnevskaya in Basov and Vishnevskaya, 1991; Schaafella nodosa Vishnevskaya in Basov and Vishnevskaya, 1991; Schaafella tochilinae Vishnevskaya in Basov and Vishnevskaya, 1991; Stichomitra tosaensis Nakaseko and Nishimura in Nakaseko et al., 1979; and Stichopilium bonum Kozlova in Kozlova and Gorbovetz, 1966.

Eostichomitra: Eostichomitra warzigita Empson-Morin, 1981; Stichomitra asymbatos Foreman, 1968; Vistularia magna Gorka, 1989; and ?Cyrtocapsa pseudacerra Tan, 1927.

Parvimitrella wallacheri (Grill and Kozur, 1986)

Figure 6.39

1986 Pseudodictyomitrella wallacheri Grill and Kozur, p. 253, pl. 4, figs. 5-7.

1986 Parvifavus irregularis Takemura, p. 9, pl. 10, figs. 10-13.

2008 Parvifavus wallacheri; Auer et al., fig. 9.48.

2011 Parvifavus irregularis; Yeh, p. 8, pl. 2, figs. 1-2. 
Remarks.-Because the paper by Grill and Kozur was published in January 1986 but the paper by Takemura in December 1986, the species name of $P$. wallacheri has priority. It is also considered the type species of this genus.

\section{Genus Pseudodictyomitrella Grill and Kozur, 1986}

Type species.—Pseudodictyomitrella spinosa Grill and Kozur, 1986.

Other species.-Parvicingula limana Cortese, 1993; Pseudodictyomitrella badouxi O'Dogherty, Goričan and Dumitrica in O'Dogherty et al., 2006; Pseudodictyomitrella escheri O'Dogherty, Goričan and Dumitrica in O'Dogherty et al., 2006; Pseudodictyomitrella renevieri O'Dogherty, Goričan and Dumitrica in O'Dogherty et al., 2006.

Remarks.-All species here included under Pseudodictyomitrella have a conical shell with an acute cephalis tapering in an apical horn. For a detailed description of all these species the reader is referred to O'Dogherty et al., 2006.

Occurrence.-Lower Bajocian to upper Bathonian.

\section{Pseudodictyomitrella limana (Cortese, 1993)} Figure 6.45-6.48

1985 Parvicingula ? sp. Yamamoto et al., p. 36, pl. 6, fig. 5.

1993 Parvicingula limana Cortese; p. 177; pl. 4, figs. 5-7.

2003 Parvicingula cappa Cortese; Suzuki and Gawlick, p. 187, fig. 6.84 only.

2006 Pseudodictyomitrella limana; O’Dogherty et al., p. 440, pl. 5, fig. 18.

Pseudodictyomitrella renevieri O'Dogherty, Goričan, and Dumitrica in O'Dogherty et al., 2006

Figure 7.5-7.7

1998 Parvicingula? sp. A1 Arakawa, pl. 10, fig. 454.

1998 Parvicingula? sp. J0 Arakawa, pl. 10, fig. 462.

2006 Pseudodictyomitrella renevieri O’Dogherty, Goričan, and Dumitrica in O'Dogherty et al., p. 440, pl. 5, figs. 19-22.

Pseudodictyomitrella sp. cf. P. renevieri O'Dogherty, Goričan, and Dumitrica in O'Dogherty et al., 2006

Figure 7.8

Remarks.-The specimen has a pointed cephalis but supposedly due to preservation it lacks the apical horn.

Genus Xitomitra new genus

Type species.—Stichomitra tairai Aita, 1987.

Other species.—Stichomitra annibill Kocher, 1981 (syn. Stichomitra? matsuokai Hull, 1997).
Diagnosis.-Shell conical with four or more segments and a large distal aperture. Very large cephalis, imperforate and usually bearing a small apical horn. Subsequent segments increasing gradually in height, with well-defined external strictures. All segments covered by a regular pattern of polygonal (mostly hexagonal) pore frames. Distalmost segments may bear thick nodes.

Etymology.-Arbitrary combination of letters (ICZN, Art. 11.3) plus mitra (from Latin, meaning cap); feminine gender.

Remarks.-Xitomitra $\mathrm{n}$. gen. differs from other genera included in this family by having a more lobate outline with well-marked strictures. It differs from Parvimitrella $\mathrm{n}$. gen. by having a thick, wider and pointed cephalic region (see pl. 3, fig. 8a of Aita, 1987).

Occurrence.-Middle Bathonian to lower Callovian.

Xitomitra annibill (Kocher, 1981) Figure 6.51-6.54

1981 Stichomitra annibill Kocher, p. 96, pl. 16, figs. 24-26.

1989 Gen 2 sp. B Hattori and Sakamoto, ? pl. 14, fig. E.

1996 Stichomitra tairai Aita; Marcucci and Prela, pl. 2, fig. 14.

1997 Stichomitra (?) matsuokai Hull, p. 164, pl. 49, figs. 2-3, 13-14, 19-20.

2003 non Stichomitra annibill; Suzuki and Gawlick, figs. 6.35-6.36. $[=X$. tairai $]$

2005 Stichomitra annibill; Šmuc and Goričan, pl. 4, fig. 24.

2006 Stichomitra annibill; O'Dogherty et al., p. 442, pl. 5, figs. $1-3$.

2006 non Stichomitra annibill; Auer et al., fig. 6.37. $[=$ X. tairai $]$

2008 non Stichomitra annibill; Auer et al., fig. 9.66. [= X. tairai $]$

2009 non Stichomitra annibill; Suzuki and Gawlick, fig. 5.16. $[=X . \quad$ tairai $]$

Remarks.-Xitomitra annibill differs from Xitomitra tairai by having a smaller cephalis and a four-segmented shell. Furthermore, the shell does not have the outer characteristic layer of ornametation as developed in the Japanese species.

\section{Xitomitra tairai (Aita, 1987)}

Figure 6.35-6.38

1987 Stichomitra (?) tairai Aita, p. 72, pl. 3, figs. 7a-9; pl. 10, figs. 3-4.

1989 Stichomitra (?) sp. cf. S. tairai; Okada et al., pl. 32, fig. 12.

1996 non Stichomitra tairai; Marcucci and Prela, pl. 2, fig. 14. $[=X$. annibill $]$

2002 Stichomitra (?) tairai; Bragin et al., fig. 6.19.

2002 Stichomitra (?) sp. aff. S. (?) takanoensis Aita; Bragin et al., fig. 6.20 .

2003 Stichomitra annibill Kocher; Suzuki and Gawlick, figs. 6.35-6.36. 
2005 Stichomitra (?) tairai; Šmuc and Goričan, pl. 4, fig. 23.

2006 Stichomitra (?) tairai; O’Dogherty et al., p. 442, pl. 5, fig. 4-5.

2006 Stichomitra annibill; Auer et al., fig. 6.37.

2008 Stichomitra annibill; Auer et al., fig. 9.66.

2009 Stichomitra annibill; Suzuki and Gawlick, fig. 5.16.

Remarks.-Xitomitra tairai is distinguished from its allied $X$. annibill by having a characteristic and delicate double layer of rhomboidal pore frames covering the post-thoracic segments. This ornamentation is specially marked on proximal segments, which fade out distally where very faint nodes tend to regularly cover the surface.

\section{Xitomitra? sp. A}

Figure 6.55

Remarks.-This very small species is characterized by proximally conical to distally subcylidrical shell covered by a doublelayered pore pattern similar to that of X. tairai. The specimen is questionably assigned to Xitomitra because its lacks the welldefined strictures and the thick large cephalis.

Order Spumellaria Ehrenberg, 1876

'Actinommids with single medulary shell'

Superfamily Actinommoidea Haeckel, 1862

Family Pantanelliidae Pessagno, 1977b

Subfamily Pantanelliinae Pessagno, 1977b

Genus Pantanellium Pessagno, 1977a

Type species._Pantanellium riedeli Pessagno, 1977a.

Occurrence.-Upper Carnian to upper Aptian.

Pantanellium riedeli Pessagno, 1977a

Figure 12.13-12.15

1977a Pantanellium riedeli Pessagno, p. 78, pl. 6, figs. 5-11.

2006 Pantanellium riedeli; O’Dogherty et al., p. 460, pl. 11, figs. 9-14. [See for complete synonymy]

Genus Gorgansium Pessagno and Blome, 1980

Type species.-Gorgansium silviesense Pessagno and Blome, 1980.

Occurrence.-Upper Norian to upper Valanginian.

Gorgansium silviesense Pessagno and Blome, 1980

Figure 12.5-12.6

1980 Gorgansium silviesense Pessagno and Blome, p. 235, pl. 11; figs. 2-3, 11, 24.

1982 Gorgansium silviesense; Mizutani and Koike, pl. 1, fig. 3.

1987 Gorgansium silviesense; Goričan, p. 182, pl. 3, fig. 4.

2006 Gorgansium silviesense; O’Dogherty et al., p. 460, pl. 11, fig. 22.
'Actinommids with multiple medulary shell'

Superfamily Pylonoidea Haeckel, 1881 emend Dumitrica, 1989

Family Dactyliosphaeridae Squinabol, 1904

Subamily Hagiastrinae Riedel, 1971

Genus Crucella Pessagno, 1971

Type species.—Crucella messinae Pessagno, 1971.

Occurrence.-Lower Carnian to upper Campanian.

Crucella theokaftensis Baumgartner, 1980

Figure 12.37

1980 Crucella theokaftensis Baumgartner, p. 308, pl. 8, figs. 19-22; pl. 12, fig. 1.

2003 Crucella theokaftensis; Suzuki and Gawlick, p. 174, fig. 6.17.

2006 Crucella theokaftensis; O’Dogherty et al., p. 464, pl. 12, fig. 16. [See for complete synonymy]

Subfamily Emiluviinae Dumitrica, 1995

Genus Emiluvia Foreman, 1973b

Neosophia Özdikmen 2009 pro Sophia Whalen and Carter in Carter et al., 1998 (preoccupied name).

Type species.—Emiluvia chica Foreman, 1973b.

Occurrence.-Lower Sinemurian to upper Valanginian.

Emiluvia salensis Pessagno, 1977a

Figure 12.39-12.40

1977a Emiluvia salensis Pessagno, p. 77, pl. 5, figs. 9-11.

1995b Emiluvia salensis; Baumgartner et al., p. 210, pl. 3215, figs. 1-3.

2006 Emiluvia salensis; O’Dogherty et al., p. 468, pl. 12, figs. 12-15. [See for complete synonymy]

Emiluvia sedecimporata (Rüst, 1885)

Figure 12.38

1885 Staurosphaera sedecimporata Rüst, p. 288, pl. 28(3), fig. 1.

1994 Emiluvia sedecimporata; Goričan, p. 67, pl. 4, fig. 4.

1995b Emiluvia sedecimporata; Baumgartner et al., p. 210, pl. 3216, figs. 1-3. [See for complete synonymy]

1997 Emiluvia sedecimporata; Chiari et al., pl.2, fig. 9.

2004 Emiluvia peteri Beccaro, p. 16, fig. 4a; pl. 2, figs. 6-8.

Emiluvia? sp.

Figure 12.41

Remarks.-This form is questionably assigned to Emiluvia. Similar forms have been assigned to Staurolonche Rüst, 1885 (e.g,. Staurolonche robusta), but this genus must be considered as nomen dubium (O'Dogherty et al., 2009). Further taxonomic 
studies are required in order to fix the taxonomic position of this species.

Family Patulibracchiidae Pessagno, 1971

Subamily Angulobracchinae Baumgartner, 1980

Genus Angulobracchia Baumgartner, 1980

Type species.—Paronaella? purisimaensis Pessagno, 1977a.

Occurrence.-Upper Aalenian to lower Albian.

Remarks.-Includes generic concept of Cavabracchia Kito and De Wever, 1992.

Angulobracchia spp.

Figure 12.28-12.30

Remarks.-These forms are very rare and badly preserved in our material; hence they have not been studied in depth in this publication.

Genus Paronaella Pessagno, 1971

Type species.-Paronaella solanoensis Pessagno, 1971.

Occurrence.-Lower Rhaetian to upper Coniacian.

Paronaella kotura Baumgartner, 1980

Figure 12.33-12.34

1980 Paronaella kotura Baumgartner, p. 302, pl. 9, figs. 15-19; pl. 12, fig. 8 .

2006 Paronaella kotura; O’Dogherty et al., p. 468, pl. 12, figs. 12-15. [See for complete synonymy]

Paronaella pristidentata Baumgartner, 1980

Figure 12.31

1980 Paronaella pristidentata Baumgartner, p. 304, pl. 9, fig. 7; pl. 12, fig. 3.

1992 Paronaella pristidentata; Steiger, p. 44, pl. 10, figs. 8-9.

1995b Paronaella pristidentata; Baumgartner et al., p. 396, pl. 3138, figs. 1-2.

1993 Paronaella cleopatrensis Pessagno, Blome, and Hull in Pesagno et al., p. 122, pl. 2, figs. 3, 25.

1997 Paronaella pristidentata; Yao, pl. 7, fig. 309.

2001 Paronaella pristidentata; Vishnevskaya, pl. 46, figs. 3-4; pl. 91, figs. 4-5.

2009 Paronaella pristidentata; Nishihara, pl. 8, fig. 186.

Subfamily Patulibracchiinae Pessagno, 1971

Genus Homoeoparonaella Baumgartner, 1980

Type species._Paronaella elegans Pessagno, 1977a.

Occurrence.-Upper Sinemurian to upper Cenomanian.
Homoeoparonaella elegans (Pessagno, 1977a)

Figure 12.32

1977a Paronaella elegans Pessagno, p. 70, pl. 1, figs. 10-11.

1995b Homoeoparonaella elegans; Baumgartner et al., p. 272, pl. 3104, figs. 1-5. [See for complete synonymy]

1999 Homoeoparonaella elegans yangi Kiessling, p. 32, pl. 6, figs. 13-14, 16.

2008 Homoeoparonaella elegans; Beccaro et al., pl. 1, fig. 22.

Homoeoparonaella spp.

Figure 12.35-12.36

Remarks.-Our specimens are badly preserved, with ray tips broken off, therefore determination at species level is not possible.

Family Pseudoaulophacinae Riedel, 1967

Genus Alievium Pessagno, 1972

Type species.-Theodiscus superbus Squinabol, 1914.

Occurrence.-Upper Bajocian to upper Maastrichtian.

Alievium? sp. aff. A. crassum (Kiessling, 1999)

Figure 12.9

1999 Tripocyclia crassa Kiessling, p. 40, pl. 8, figs. 14, 22.

Remarks.-Our specimen has shorter spines and somewhat larger nodes on the surface than the Antarctic specimens. The genus is queried because the name Tripocyclia is not valid (nomen dubium see O'Dogherty et al., 2009, p. 288) and these forms belong to a new genus not described yet.

\section{Alievium? longispineum Yang and Wang, 1990} Figure 12.7-12.8

1990 Alievium longispineum Yang and Wang, p. 204, pl. 2, figs. 2, 4 .

Remarks.-This form has high generic affinity with the previous species and the genus is queried for the same reasons.

Family Tritrabidae Baumgartner, 1980

Genus Tritrabs Baumgartner, 1980

Type species.-Paronaella? casmaliaensis Pessagno, 1977a.

Occurrence.-Lower Aalenian to lower Aptian.

Tritrabs ewingi (Pessagno, 1971)

Figure 12.24-12.27

1971 Paronaella (?) ewingi Pessagno, p. 47, pl. 19, figs. 2-5.

2006 Tritrabs ewingi; O’Dogherty et al., p. 472, pl. 11, figs. 37, 43. [See for complete synonymy] 
Superfamily Sponguroidea Haeckel, 1862

Family Archaeospongoprunidae Pessagno, 1973

Genus Archaeospongoprunum Pessagno, 1973

Type species.-Archaeospongoprunum venadoensis Pessagno, 1973.

Occurrence.-Upper Permian to upper Campanian.

Archaeospongoprunum elegans $\mathrm{Wu}, 1993$

Figure 12.10-12.12

1993 Archaeospongoprunum elegans, p 118, pl. 1, figs. 5, 7, 23.

1997 Archaeospongoprunum aff. elegans Wu; Hull, p. 27, pl. 8, figs. 4, 9, 18-19.

2001 Archaeospongoprunum sp. Nishizono, pl. 1, fig. 3.

2002 Archaeospongoprunum elegans; Beccaro et al., pl. 1, fig. 22.

2006 Archaeospongoprunum elegans; O'Dogherty et al., p. 472 , pl. 11, figs. $24-25$.

Remarks.-Archaeospongoprunum elegans differs from A. imlayi Pessagno, by its subrectangular shape and massive spines with prominent subsidiary grooves. We considered specimens showing slightly torsioned spines distally to be included in this species (see Fig. 12.11-12 and Hull's 1997 material).

Family Bernoulliidae Pessagno, Blome, and Hull in Pessagno et al., 1993

Genus Bernoullius Baumgartner, 1984

Type species.—Eucyrtis? dicera Baumgartner in Baumgartner et al., 1980.

Occurrence.-Lower Toarcian to lower Aptian.

Bernoullius cristatus Baumgartner, 1984

Figure 12.4

1984 Bernoullius cristatus Baumgartner, p. 760, pl. 2, figs. 14-15.

1993 Bernoullius cristatus; Pessagno et al., p. 119, pl. 1, fig. 14.

1995b Bernoullius cristatus; Baumgartner et al., p.122, pl. 3221, figs. 1-3.

2003 Bernoullius cristatus; Suzuki and Gawlick, p. 172, fig. 5.10. [See for complete synonymy]

Superfamily Saturnaloidea Deflandre, 1953

Family Saturnalidae Deflandre, 1953

Subfamily Hexasaturnalinae Kozur and Mostler, 1983

Genus Hexasaturnalis Kozur and Mostler, 1983

Type species.—Spongosaturnalis ? hexagonus Yao 1972.

Occurrence.-Middle Toarcian to upper Valanginian.
Remarks.-Includes generic concepts of Kozurastrum De Wever, 1984 and Yaosaturnalis Kozur and Mostler, 1983.

Hexasaturnalis minor (Baumgartner in Baumgartner et al., 1995b)

Figure 12.23

1995b Acanthocircus suboblongus minor Baumgartner in Baumgartner et al., p. 66, pl. 3085, only fig. 1-3, not fig. 4 (H. nakasekoi)

2005 Hexasaturnalis minor; Dumitrica and Dumitrica-Jud, pl. 2, figs. 6, 9-13.

2008 Hexasaturnalis minor; Beccaro et al., pl. 1, fig. 18.

Hexasaturnalis nakasekoi Dumitrica and Dumitrica-Jud 2005 Figure 12.19-12.20

2005 Hexasaturnalis nakasekoi Dumitrica and DumitricaJud, p. 161, pl. 1, figs. 3-13; pl. 2, figs. 1-4, 7-8. [See for complete synonymy]

2006 Hexasaturnalis nakasekoi; O’Dogherty et al., p. 473, pl. 12, fig. 31. [See for complete synonymy]

2008 Hexasaturnalis minor; Beccaro et al., pl. 1, fig. 19.

Hexasaturnalis suboblongus (Yao, 1972)

Figure 12.21-12.22

1972 Spongosaturnalis (?) suboblongus Yao, p. 29, pl. 3, figs. 1-6; pl. 10, figs. 3a-c.

1995b Acanthocircus suboblongus suboblongus; Baumgartner et al., p. 68, pl. 3088, fig. 2-4, non fig $1[=H$. nakasekoi].

2005 Hexasaturnalis suboblongus; Dumitrica and DumitricaJud, pl. 1, figs. 1-2.

2013 Hexasaturnalis suboblongus; Chiari et al., p. 411, fig. 12r.

Hexasaturnalis tetraspinus (Yao, 1972)

Figure 12.16-12.18

1972 Spongosaturnalis? tetraspinus Yao, p. 29, pl. 4, figs. 1-6; pl. 11, figs. 1-2.

1995b Hexasaturnalis tetraspinus (Yao); Baumgartner et al., p. 254, pl. 3089, figs. 1-3.

2006 Hexasaturnalis tetraspinus; Goričan et al., p. 190, pl. 3089, figs. 1-5. [See for complete synonymy]

2013 Hexasaturnalis tetraspinus; Chiari et al., fig. 12s.

Table 2. Assigned ages and zones of studied samples.

\begin{tabular}{lccl}
\hline Locality & Sample Number & UAZone & Assigned Age \\
\hline EW & 158 & 5 & latest Bajocian-early Bathonian \\
BMW & 28 & 5 & latest Bajocian-early Bathonian \\
BMW & 32 & 7 & late Bathonian-early Callovian \\
BMW & 34 & 7 & late Bathonian-early Callovian \\
BMW & 31 & $5-7$ & latest Bajocian-early Callovian \\
BMW & 30 & $8-9$ & middle Callovian-late Oxfordian \\
BMW & 35 & $9-11$ & middle Oxfordian-late Kimmeridgian \\
BMW & 33 & 12 & early-early late Tithonian \\
BMW & 26 & 5 & latest Bajocian-early Bathonian \\
BMW & $13 b$ & 5 & latest Bajocian-early Bathonian \\
BMW & $13 \mathrm{c}$ & 8 & middle Callovian-early Oxfordian \\
\hline
\end{tabular}




\section{Age of radiolarian samples}

In this section, we present the radiolarian age and results obtained for the Hallstatt Mélange in four localities at Bad Mittendorf area (Table 2). Only the stratigraphically most important species are discussed. For the complete inventory of all samples, see Table 1 .

Kumitzberg.-In this area, the massive dark-gray radiolarite beds (sample EW-158) are intercalated by thin layers of cherty shales. The co-occurrence of Unuma latusicostatus (Aita) with Bernoullius cristatus Baumgartner (Table 1) suggests assignment to UAZ 5 (latest Bajocian-early Bathonian) of Baumgartner et al. (1995a). Pantanellium riedeli Pessagno, which first appears in UAZ 7, was also found. This species has a very large variability that overlaps with other Pantanellium species. Its range is thus ignored in the age determination.

Steinwand north.-In this area two sections accurately determine the age of the radiolarite succession. The most complete section, in the northeastern part of the syncline structure (Fig. 4), records continuous radiolarite deposition from Bathonian to the Oxfordian. The lowermost part of the radiolarite succession (near the entrance of the valley, sample BMW-28, Fig. 4) yielded a radiolarian assemblage of latest Bajocian-early Bathonian age (UAZ 5 of Baumgartner et al., 1995a), defined on the co-occurrence of Protunuma ochiensis Matsuoka with Unuma latusicostatus (Aita). The conflicting range of Pantanellium riedeli Pessagno (UAZ 7-12) was ignored for the same reason as in the aforementioned sample EW-158 from Kumitzberg.

The youngest part of the succession is preserved in the core of the syncline (sample BMW-35, Fig. 4) and yielded a radiolarian assemblage of middle-late Oxfordian to late Kimmeridgian-early Tithonian age (UAZs 9-11 of Baumgartner et al., 1995a), based on the occurrence of Archaeodictyomitra minoensis (Mizutani), Zhamoidellum ovum Dumitrica, and Emiluvia sedecimporata (Rüst). The interval between these two ages was also recognized. UAZ 7 (late Bathonian-early Callovian) or UAZ 8 (middle Callovian-early Oxfordian) was determined in samples BMW-32 and BMW-34. Striatojaponocapsa conexa (Matsuoka) in sample BMW-32 and Preawilliriedellum robustum (Matsuoka) in sample BMW-34 suggest that these two samples are not younger than UAZ 7, but Gongylothorax favosus Dumitrica, which first occurs in UAZ 8 is also associated.

At the end of the valley is located the second stratigraphic section outcropping in this area. The radiolarian assemblage in the youngest investigated sample (BMW-33) indicates a Tithonian age. Early-early late Tithonian UAZ 12 is inferred from the co-occurrence of Eucyrtidiellum pyramis (Aita in Aita and Okada) with Protunuma japonicus Matsuoka and Yao.

Area between Krautmoos and Mischenirwiese.-The thick succession of mass-flow deposits in this area is dated by the assemblages studied in the intercalated radiolarite matrix. The radiolarite sample BMW-26 collected below the first debris-flow deposit is dated latest Bajocian-early Bathonian age (UAZ 5 of Baumgartner et al., 1995a). This age is constrained with Semihsuum amabile (Aita), Saitoum trichylum De Wever, and Mizukidella kamoensis (Mizutani and Kido). The same age (UAZ 5) was obtained from a chert clast (sample BMW-13b) in the upper part of the succession. The age-diagnostic species in this clast are Theocapsomella cordis (Kocher), Eucyrtidiellum pustulatum Baumgartner, and Unuma latusicostatus (Aita). The matrix between the chert clasts (sample BMW-13c) yielded a significantly younger radiolarian fauna of middle Callovianearly Oxfordian age (UAZ 8 of Baumgartner et al., 1995a), as indicated by co-occurrence of Spinosicapsa spinosa (Ožvoldová) with Hemicryptocapsa marcucciae (Cortese).

As proven by radiolarian dating, the resedimentation of the Hallstatt Limestone started in the region of Bad Mitterndorf area since the Bathonian and prevailed at least until the Oxfordian. The area of the Hallstatt Limestone mass flows and slide blocks is separated from the coeval radiolarite succession without mass-flow deposits (the Steinwand section) by a younger thrust or fault.

\section{Discussion}

The time span of deposition of the radiolarite basin, which contains the far-traveled Hallstatt Limestone blocks, is determined as Bathonian to Oxfordian. Redeposition started in the ?late Bathonian and ended in the Oxfordian, as proven by the radiolarite matrix age and the age of the overlying sediments (O'Dogherty and Gawlick, 2008). Radiolarian ages and component spectrum define this redeposit as part of the Sandlingalm Basin (Sandlingalm Formation: Fig. 2), one of the oldest basins formed in a relative early stage of compression of the Neotethys (see Gawlick et al., 2007a, 2009, 2012; Missoni and Gawlick, $2011 \mathrm{~b}$ for details).

The depositional areas of the radiolarites of the section Steinwand-Mischenirwiese (Fig. 4) mass flows are missing. In the more northern areas of the Northern Calcareous Alps (Fig. 3), radiolarite deposition starts relatively early, indicating an early deepening event due to the tectonic load of the advancing Hallstatt nappes. First imbricates started to form in the BathonianCallovian (I and II in Fig. 5), as indicated by age dating of the matrix radiolarites. In the Oxfordian (III-IIIa in Fig. 5) the mass flows also contain components of the older radiolarite "mélange" basins and the section Mischenirwiese attained a more basinal position, as indicated by the sedimentological features of the radiolarite succession. In the late Oxfordian the nappe front reached the depositional area of the Mischenirwiese section, as shown in the overlying mélange (IV in Fig. 5). In the Kimmeridgian-Tithonian, nappe propagation stopped and the nappe stack was sealed by a carbonate platform. In the Kimmeridgian, coarse reefal debris was shed into this deep-water basin (ODogherty and Gawlick, 2008) with a general fining-upward trend. Due to uplift and demise of the southern Platform in the late Tithonian (Fig. 5; Gawlick and Missoni 2011a), only fine-grained siliceous limestones were deposited in the basin, as proven with radiolarian dating in this study (see the youngest radiolarian sample of the Steinwand locality).

\section{Acknowlegments}

This work is a contribution of the research projects P14131TEC, P15060, and P16812 (Austrian Science Foundation FWF 
projects), research program P1-0008 (Slovenian Research Agency), and CGL2011-23759 (Spanish Ministry of Science and Technology). We are greatly indebted to Elizabeth S. Carter for her positive comments and careful review. Steve Hageman, editor of the Journal, is also gratefully acknowledged for his suggestions and comments improving the final version of this paper.

\section{References}

Adachi, M., 1982, Some considerations on the Mirifusus baileyi assemblage in the Mino terrain, central Japan: News of Osaka Micropaleontologists, special volume, v. 5, p. 211-225.

Aita, Y., 1982, Jurassic radiolarian biostratigraphy in Irazuyama district, Kochi Prefecture, Japan-a preliminary report: News of Osaka Micropaleontologists, special volume, v. 5, p. 255-270.

Aita, Y., 1985, Jurassic radiolarian biostratigraphy of the Irazuyama Formation (Takano Section), Shikoku, Japan: Scientific and Technical Reports of the Mining College, Akita University, v. 6, p. 33-41.

Aita, Y., 1986, Radiolaria, in Aita, Y., and Okada, H., Radiolarians and calcareous nannofossils from the uppermost Jurassic and Lower Cretaceous strata of Japan and Tethyan regions: Micropaleontology, v. 32, p. $97-128$.

Aita, Y., 1987, Middle Jurassic to Lower Cretaceous radiolarian biostratigraphy of Shikoku with reference to selected sections in Lombardy Basin and Sicily: Science Reports of the Tohoku University, Series 2: Geology, v. 58 , p. $1-91$.

Aita, Y., and Okada, H., 1986, Radiolarians and calcareous nannofossils from the uppermost Jurassic and Lower Cretaceous strata of Japan and Tethyan regions: Micropaleontology, v. 32, p. 97-128.

Aliev, K.S., 1967, New radiolarian species of the Valanginian and Albian stages of northeastern Azerbaidzhan, in Aliev, M.M., ed., Melovye Otlozheniya Vostochnogo Kavkaza i Prilegayushchikh Oblastei: Moscow, Nauka, p. 23-30. [In Russian]

Arakawa, R., 1997, Jurassic radiolarian succession from the siliceous mudstone in the Kuzuu area of the Ashio Terrane, central Japan. Part I: Bulletin of the Tochigi Prefectural Museum, v. 14, p. 1-19.

Arakawa, R., 1998, Middle Jurassic Radiolaria assemblages from Manganese dioxide nodules, Kuzuu area of Ashio terrane, central Japan: Bulletin of the Tochigi Prefectural Museum, v. 15, p. 51-76.

Aubrecht, R., and Ožvoldová, L., 1994, Middle Jurassic-Lower Cretaceous development of the Pruské Units in the western part of the Pieniny Klippen Belt: Geologica Carpathica, v. 45, p. 211-223.

Auer, M., Gawlick, H.-J., and Suzuki, H., 2006, Die Unter-Oxford-RadiolaritMegabrekzie am Nordrand des Dachstein-Blockes (Nördliche Kalkalpen, Österreich): Radiolarienfaunen, Mikrofazies des Komponentenbestandes und tektonische Bedeutung: Jahrbuch der geologischen Bundesanstalt, Wien, v. 146, p. 33-51.

Auer, M., Gawlick, H.-J., Suzuki, H., and Schlagintwei, F., 2008, Spatial and temporal development of siliceous basin and shallow-water carbonate sedimentation in Oxfordian Northern Calcareous Alps: Facies, v. 55, p. $63-87$.

Bak, M., 1999, Uppermost Maastrichtian Radiolaria from the Magura Nappe deposits, Czech Outer Carpathians: Annales Societatis Geologorum Poloniae, v. 69, p. 137-159.

Bandini, A.N., Baumgartner, P.O., Flores, K., Dumitrica, P., Hochard, C., Stampfli, G., and Jackett, S.-J., 2011, Aalenian to Cenomanian Radiolaria of the Bermeja Complex (Puerto Rico) and Pacific origin of radiolarites on the Caribbean Plate: Swiss Journal of Geosciences, v. 104, p. 367-408.

Basov, V.A., and Vishnevskaya, V.S., 1991, Upper Mesozoic Stratigraphy of the Pacific Ocean: Circum-Pacific Council for Energy and Mineral Resources, Moscow, Nauka, 200 p. [In Russian]

Baumgartner, P.O., 1980, Late Jurassic Hagiastridae and Patulibracchiidae (Radiolaria) from the Argolis Peninsula (Peleponnesus, Greece): Micropaleontology, v. 26, p. 274-322.

Baumgartner, P.O., 1984, A Middle Jurassic-Early Cretaceous low latitude radiolarian zonation based on unitary associations and age of Tethyan radiolarites: Eclogae Geologicae Helvetiae, v. 77, p. 729-841.

Baumgartner, P.O., 1995, Acanthocircus suboblongus minor, Baumgartner, nov. subsp., in Baumgartner, P.O., O'Dogherty, L., Goričan, Š., Dumitrica-Jud, R., Dumitrica, P., Pillevuit, A., Urquhart, E., Matsuoka, A., Danelian, T., Bartolini, A., Carter, E.S., De Wever, P., Kito, N., Marcucci, M., and Steiger, T., Radiolarian catalogue and systematics of Middle Jurassic to Early Cretaceous Tethyan genera and species: Mémoires de Géologie (Lausanne), v. 23, p. 66.
Baumgartner, P.O., 1995, Acanthocircus suboblongus minor, Baumgartner, nov. subsp., in Baumgartner, P.O., O’ Dogherty, L., Goričan, Š., Dumitrica-Jud, R., Dumitrica, P., Pillevuit, A., Urquhart, E., Matsuoka, A., Danelian, T., Bartolini, A., Carter, E.S., De Wever, P., Kito, N., Marcucci, M., and Steiger, T., Radiolarian catalogue and systematics of Middle Jurassic to Early Cretaceous Tethyan genera and species: Mémoires de Géologie (Lausanne), v. 23 , p. 642 .

Baumgartner, P.O., and Bernoulli, D., 1976, Stratigraphy and radiolarian fauna in a Late Jurassic-Early Cretaceous section near Achladi (Evvoia, Eastern Greece): Eclogae Geologicae Helvetiae, v. 69, p. 601-626.

Baumgartner, P.O., De Wever, P., and Kocher, R., 1980, Correlation of Tethyan Late Jurassic-Early Cretaceous radiolarian events: Cahiers de Micropaléontologie, part 2, p. 23-86.

Baumgartner, P.O., Bartolini, A., Carter, E.S., Conti, M., Cortese, G., Danelian, T., De Wever, P., Dumitrica, P., Dumitrica-Jud, R., Goričan, Š., Guex, J., Hull, D.M., Kito, N., Marcucci, M., Matsuoka, A., Murchey, B., O’Dogherty, L., Savary, J., Vishnevskaya, V., Widz, D., and Yao, A., 1995a, Middle Jurassic to Early Cretaceous radiolarian biochronology of Tethys based on Unitary Associations, in Baumgartner, P.O., O'Dogherty, L., Goričan, Š., Urquhart, E., Pillevuit, A., and De Wever, P., eds., Mémoires de Géologie (Lausanne), v. 23, p. 1013-1048.

Baumgartner, P.O., O’Dogherty, L., Goričan, Š., Dumitrica-Jud, R., Dumitrica, P., Pillevuit, A., Urquhart, E., Matsuoka, A., Danelian, T., Bartolini, A., Carter, E.S., De Wever, P., Kito, N., Marcucci, M., and Steiger, T., 1995b, Radiolarian catalogue and systematics of Middle Jurassic to Early Cretaceous Tethyan genera and species, in Baumgartner, P.O., O'Dogherty, L., Goričan, S., Urquhart, E., Pillevuit, A., and De Wever, P., eds., Mémoires de Géologie (Lausanne), v. 23, p. 37-685.

Baumgartner, P.O., Flores, K., Bandini, A.N., Girault, F., and Cruz, D., 2008 , Upper Triassic to Cretaceous radiolaria from Nicaragua and Northern Costa Rica-The Mesquito Composite Oceanic Terrane: Ofioliti, v. 33, p. 1-19.

Beccaro, P., 2004, New Middle and Upper Jurassic Radiolaria from western Sicily and Southern Alps (Italy): Razprave IV, Razreda SAZU, v. 45 , p. $6-27$.

Beccaro, P., Baumgartner, P.O., and Martire, L., 2002, Radiolarian biostratigraphy of the Fonzaso Formation, Middle-Upper Jurassic, Southern Alps, Italy: Micropaleontology, v. 48, p. 43-60.

Beccaro, P., Diserens, M.-O., Goričan, Š., and Martire, L., 2008, Callovian radiolarians from the lowermost Calcare Selcifero di Fonzaso at Ponte Serra (Trento Plateau, Southern alps, Italy): Rivista italiana di Paleontologia e Stratigrafia, v. 114, p. 489-504

Bernoulli, D., and Jenkyns, H.C., 1974, Alpine, Mediterranean and Central Atlantic Mesozoic facies in relation to the early evolution of the Tethys, in Dott, R.H., and Shaver, R.H., eds., Modern and Ancient Geosynclinal Sedimentation, a Symposium: Special Publication of the Society of Economic Paleontologists and Mineralogists, v. 19, p. 129-160.

Bernoulli, D., and Jenkyns, H.C., 2009, Ancient oceans and continental margins of the Alpine-Mediterranean Tethys: deciphering clues from Mesozoic pelagic sediments and ophiolites: Sedimentology, v. 56, p. 149-190.

Blome, C., 1984, Middle Jurassic (Callovian) radiolarians from carbonate concretions, Alaska and Oregon: Micropaleontology, v. 30, p. 343-389.

Böhm, F., 1992, Mikrofazies und Ablagerungsmilieu des Lias und Dogger der Nordöstlichen Kalkalpen: Erlanger Geologische Abhandlungen, v. 121, p. 57-217.

Böhm, F., 2003, Lithostratigraphy of the Adnet Group (Lower to Middle Jurassic, Salzburg, Austria), in Piller W.E., ed., Stratigraphia Austriaca: Österreichische Akademie der Wissenschaften Schriftenreihe der erdwissenschaftlichen Kommission, v. 16, p. 231-268.

Böhm, F., Dommergues, J.L., and Meister, C., 1995, Breccias of the Adnet Formation: indicators of a Mid-Liassic event in the Northern Calcareous Alps (Salzburg/Austria): Geologische Rundschau, v. 84, p. 272-286.

Bragin, N.Y., and Tekin, U.K., 2002, Stichomitra (?) angulata Bragin and Tekin, nov. sp., in Bragin, N.Y., Tekin, U.K., and Özçelik, Y., Middle Jurassic radiolarians from the Akgöl Formation, central Pontids, northern Turkey: Neues Jahrbuch für Geologie und Paläontologie, Monatshefte, v. 10, p. 618.

Bragin, N.Y., Tekin, U.K., and Özçelik, Y., 2002, Middle Jurassic radiolarians from the Akgöl Formation, central Pontids, northern Turkey: Neues Jahrbuch für Geologie und Paläontologie, Monatshefte, v. 10, p. 609-628.

Campbell, A.S., and Clark, B.L., 1944, Radiolaria from Upper Cretaceous of Middle California: Geological Society of America, Special Papers, v. 57, p. 1-61.

Carter, E.S., 2006, Praeparvicingula tlellensis Carter, nov. sp., in Goričan, Š., Carter, E.S., Dumitrica, P., Whalen, P.A., Hori, R. S., De Wever, P., O'Dogherty, L., Matsuoka, A., and Guex, J. Catalogue and systematics of Pliensbachian, Toarcian and Aalenian radiolarian genera and species: Ljubljana, Slovenia, ZRC Publishing, Scientific Research Centre of the Slovenian Academy of Sciences and Arts, p. 340. 
Carter, E.S., Whalen, P.A., and Guex, J., 1998, Biochronology and paleontology of Lower Jurassic (Hettangian and Sinemurian) radiolarians, Queen Charlotte Islands, British Columbia: Geological Survey of Canada, Bulletin, v. 496, p. 1-162.

Cayeux, L., 1897, Contribution à l'étude micrographique des terrains sédimentaires. 1. Etude de quelques dépôts siliceux secondaires et tertiaires du Bassin de Paris et de la Belgique. 2. Craie du Bassin de Paris: Mémoires de la Société Géologique du Nord, Lille, v. 4, p. 1-591.

Chiari, M., Cortese, G., and Marcucci, M., 1997, Radiolaria, in Chiari, M., Cortese, G., Marcucci, M., and Nozzoli, N., Radiolarian biostratigraphy in the sedimentary cover of the ophiolites of South-Western Tuscany, Central Italy: Eclogae geologicae Helvetiae, v. 90, p. 55-77.

Chiari, M., Cortese, G., Marcucci, M., and Nozzoli, N., 1997, Radiolarian biostratigraphy in the sedimentary cover of the ophiolites of South-Western Tuscany, Central Italy: Eclogae Geologicae Helvetiae, v. 90, p. 55-77.

Chiari, M., Marcucci, M., and Prela, M., 2002, New species of Jurassic radiolarians in the sedimentary cover of ophiolites in the Mirdita area, Albania: Micropaleontology, v. 48, p. 61-87.

Chiari, M., Marcucci, M., and Prela, M., 2004, Radiolarian assemblages from the Jurassic cherts of Albania: new data: Ofioliti, v. 29, p. 95-105.

Chiari, M., Baumgartner, P.O., Bernoulli, D., Bortolotti, V., Marcucci, M., Photiades, A., and Principi, G., 2013, Late Triassic, Early and Middle Jurassic Radiolaria from ferromanganese-chert 'nodules' (Angelokastron, Argolis, Greece): evidence for prolonged radiolarite sedimentation in the Maliac-Vardar Ocean: Facies, v. 59, p. 391-424.

Conti, M., 1986, New data on the biostratigraphy of the Tuscan cherts at Monte Cetona (Southern Tuscany, Italy): Marine Micropaleontology, v. 11, p. 107-112.

Cordey, F., 1998, Radiolaires des complexes d'accrétion de la Cordillère Canadienne (Colombie-Britannique): Geological Survey of Canada, Bulletin, v. 509, p. 1-209.

Cortese, G., 1993, Radiolarian biostratigraphy of the Tuscan Cherts (Tuscan Succession) from Val di Lima, Tuscany, Northern Apennines): Palaeopelagos Journal, v. 3, p. 165-185

Danelian, T., Lahsini, S., and De Rafélis, M., 2006, Upper Jurassic Radiolaria from the Vocontian basin of SE France: Eclogae Geologicae Helvetiae, v. 99 (Supplement 1), p. S35-S47.

Danelian, T., Asatryan, G., Sosson, M., Person, A., Sahakyan, L., and Galoyan, G., 2008, Discovery of Middle Jurassic (Bajocian) Radiolaria from the sedimentary cover of the Vedi ophiolite (Lesser Caucasus, Armenia) Comptes Rendus Palevol, v. 7, p. 327-334.

De Wever, P., 1981, Une nouvelle sous-famille, les Poulpinae, et quatre nouvelles espèces de Saitoum radiolaires mésozoïques tethysiens: Geobios, v. 14 , p. $5-15$.

De Wever, P., 1984, Révision des radiolaires Mésozoïque de type Saturnalide, proposition d'une nouvelle classification: Revue de Micropaléontologie, v. 27 , p. $10-19$.

De Wever, P., and Caby, R., 1981, Datation de la base des schistes lustrés postophiolitiques par des radiolaires (Oxfordien supérieur-Kimmeridgien moyen) dans les Alpes Cottiennes (Saint-Véran, France): Comptes Rendus de l'Académie des Sciences de Paris, Série II, v. 292, p. 467-472.

De Wever, P., and Miconnet., P., 1985, Datations directes des radiolarites du bassin du Lagonero (Lucanie, Italie méridionale). Implications et conséquences: Revista Española de Micropaleontología, v. 17, p. 373-402.

De Wever, P., Dumitrica, P., Caulet, J.P., and Caridroit, M., 2001, Radiolarians in the Sedimentary Record, Amsterdam, Gordon and Breach Science Publishers, $533 \mathrm{p}$.

Deflandre, G., 1953, Radiolaires fossiles, in Grassé, P.P., ed., Traité de Zoologie 1: Paris, Masson, p. 389-436.

Diersche, V., 1980, Die Radiolarite des Oberjura im Mittelabschnitt der Nördlichen Kalkalpen: Geotektonic Forschung, v. 58, p. 1-217.

Djerić, N., Schmid, S.M., and Gerzina, N., 2012, Middle Jurassic radiolarian assemblages from the sedimentary cover of the Adriatic margin (Zlatar Mountain, SW Serbia): Bulletin de la Societe Geologique de France, v. 183, p. $359-368$

Dumitrica, P., 1970, Cryptocephalic and cryptothoracic Nassellaria in some Mesozoic deposits of Romania: Revue Roumaine de Géologie, Géophysique et Géographie (série Géologie), v. 14, p. 45-124.

Dumitrica, P., 1982, Genus Cinguloturris Dumitrica, nov. gen., in Dumitrica, P., and Mello, J., On the age of the Meliata Group and the Silica Nappe radiolarites (localities Drzkovce and Bohunovo, Slovak Karst, CSSR) Geologicke prace, Zpravy, v. 77, p. 22.

Dumitrica, P., 1989, Internal skeletal structures of the superfamily Pyloniacea (Radiolaria), a basis of a new systematics: Revista Española de Micropaleontología, v. 21, p. 207-264

Dumitrica, P., 1995, Systematic framework of Jurassic and Cretaceous Radiolaria, in Baumgartner, P.O., O’Dogherty, L., Goričan, Š., Urquhart, E., Pillevuit, A., and De Wever, P., eds., Middle Jurassic to Lower Cretaceous
Radiolaria of Tethys: occurrences, systematics, biochronology: Mémoires de Géologie (Lausanne), v. 23, p. 19-35.

Dumitrica, P., 1997, Radiolaria, in Dumitrica, P., Immenhauser, A., and Dumitrica-Jud, R., Mesozoic Radiolarian Biostratigraphy from Masirah Ophiolite, Sultanate of Oman Part I: Middle Triassic, Uppermost Jurassic and Lower Cretaceous Spumellarians and Multisegmented Nassellarians: Bulletin of the national Museum of Natural Science, Taiwan, v. 9, p. 1-106.

Dumitrica, P., 2006, Zhamoidellum yehae Dumitrica, nov. sp., in Goričan, Š., Carter, E.S., Dumitrica, P., Whalen, P.A., Hori, R.S., De Wever, P., O’Dogherty, L., Matsuoka, A., and Guex, J. Catalogue and systematics of Pliensbachian, Toarcian and Aalenian radiolarian genera and species, Ljubljana, Slovenia, ZRC Publishing, Scientific Research Centre of the Slovenian Academy of Sciences and Arts, p. 412.

Dumitrica, P., and Dumitrica-Jud, R., 2005, Hexasaturnalis nakasekoi nov. sp., a Jurassic saturnalid radiolarian species frequently confounded with Hexasaturnalis suboblongus (Yao): Revue de Micropaléontologie, v. 48 , p. $159-168$.

Dumitrica, P., and Mello, J., 1982, On the age of the Meliata Group and the Silica Nappe radiolarites (localities Drzkovce and Bohunovo, Slovak Karst, CSSR): Geologicke Prace, Zpravy, v. 77, p. 17-28.

Dumitrica, P., and Zügel, P., 2003, Lower Tithonian mono- and dicyrtid Nassellaria (Radiolaria) from the Solnhofen area (southern Germany): Geodiversitas, v. 25, p. 5-72

Dumitrica, P., Immenhauser, A., and Dumitrica-Jud, R., 1997, Mesozoic Radiolarian Biostratigraphy from Masirah Ophiolite, Sultanate of Oman Part I: Middle Triassic, Uppermost Jurassic and Lower Cretaceous Spumellarians and Multisegmented Nassellarians: Bulletin of the National Museum of Natural Science, Taiwan, v. 9, p. 1-106.

Ebli, O., 1997, Sedimentation und Biofazies an passiven Kontinentalrandern: Lias und Dogger des Mittelabschnitts der Nordlichen Kalkalpen und des fruhen Atlantik (DSDP site 547B, offshore Marokko): Münchner geowissenschaftliche Abhandlungen. Reihe A, Geologie und Paläontologie, v. 32 , p. $1-255$.

Ehrenberg, C.G., 1838, Über die Bildung der Kreidefelsen und des Kreidemergels durch unsichtbare Organismen: Abhandlungen der Königlichen Akademie des Wissenschaften zu Berlin Jahrgang, v. 1838, p. 59-147.

Ehrenberg, C.G., 1876, Fortsetzung der mikrogeologischen Studien als Gesamtübersicht der mikroskopischen Paläontologie gleichartig analysirter Gebirgsarten der Erde, mit specieller Rücksicht auf den PolycystinenMergel von Barbados: Abhandlungen der Königlichen Akademie der Wissenschaften zu Berlin Jahrgang, v. 1876, p. 1-225.

El Kadiri, K., 1992, Description de nouvelles espèces de radiolaires jurassiques de la Dorsale calcaire externe (Rif, Maroc): Revista Española de Paleontología, Número Extraordinario 3 (Ejemplar dedicado a: VI Jornadas de Paleontología, Granada 1990), p. 37-48.

Empson-Morin, K., 1981, Campanian Radiolaria from DSDP Site 313, Mid-Pacific Mountains: Micropaleontology, v. 27, p. 249-292.

Faupl, P., and Wagreich, M., 2000, Late Jurassic to Eocene palaeogeography and geodynamic evolution of the Eastern Alps: Mitteilungen der Österreichischen Geologischen Gesellschaft, v. 92, p. 79-94.

Fischli, H., 1916, Beitrag zur Kenntnis der fossilen Radiolarien in der Riginagelfluh: Mitteilungen der Naturwissenschaftlichen Gesellschaft in Winterthur, Jahrgang 1915-1916, v. 11, p. 44-47.

Foreman, H.P., 1968, Upper Maestrichtian Radiolaria of California: Special Papers in Palaeontology, v. 3, p. 1-82

Foreman, H.P., 1973a, Radiolaria of Leg 10 with systematics and ranges for the families Amphipyndacidae, Artostrobiidae and Theoperidae, in Worzel, J.L., Bryant, W., Beall Jr., A.O., Capo, R., Dickinson, K. Foreman, H.P., Laury, R., McNeely, B.W., and Smith, L.A., eds., Initial Reports of the Deep Sea Drilling Project, Volume 10: Washington, DC, U.S. Government Printing Office, p. 407-474.

Foreman, H.P., 1973b, Radiolaria from DSDP Leg 20, in Heezen, B.C., MacGregor, J.D., Foreman, H.P., Forristall, G., Hekel, H., Hesse, R., Hoskins, R.H., Jones, E.J.W., Kaneps, A.G., Krasheninnikov, V.A., Okada, H., and Ruef, M.H., eds., Initial Reports of the Deep Sea Drilling Project, Volume 20: Washington, DC, U.S. Government Printing Office, p. 249-305.

Frisch, W., 1979, Tectonic Progradation on plate tectonic evolution of the Alps: Tectonophysics, v. 60 , p. $121-139$

Frisch, W., and Gawlick, H.-J., 2003, The nappe structure of the central Northern Calcareous Alps and its disintegration during Miocene tectonic extrusiona contribution to understanding the orogenic evolution of the Eastern Alps: International Journal of Earth Sciences, v. 92, p. 712-727.

Gawlick, H.-J., 1996, Die früh-oberjurassischen Brekzien der Strubbergschichten im Lammertal-Analyse und tektonische Bedeutung (Nördliche Kalkalpen, Österreich): Mitteilungen der Gesellschaft der Geologie und Bergbaustudenten in Österreich, v. 39/40, p. 119-186.

Gawlick, H.-J., 1997, Conodont Colour Alteration Indizes (CAI) - eine Möglichkeit für die Kartierung des Öl- und Gasfensters in mehrphasig 
deformierten karbonatdominierten Sedimentbecken: Erdöl Erdgas Kohle, v. 113 , p. $164-167$.

Gawlick, H.-J., and Frisch, W., 2003, The Middle to Late Jurassic carbonate clastic radiolaritic flysch sediments in the Northern Calcareous Alps: sedimentology, basin evolution and tectonics-an overview: Neues Jahrbuch für Geologie und Paläontologie, Abhandlungen, v. 230, p. $163-213$.

Gawlick, H.-J., and Schlagintweit, F., 2006, Berriasian drowning of the Plassen carbonate platform at the type-locality and its bearing on the early Eoalpine orogenic dynamics in the Northern Calcareous Alps (Austria): International Journal of Earth Sciences, v. 95, p. 451-462.

Gawlick, H.-J., and Suzuki, H., 2004, Radiolaria, in Gawlick, H.-J., Schlanginweit, F., Ebli, O., and Suzuki, H., Die Plassen-Formation (Kimmeridgium) des Krahstein (Steirisches Salzkammergut, Östereich) und ihre Unterkagerung: neue Daten zur Fazies, Biostratigraphie und Sedimentologie: Zentralblatt für Geologie und Paläontologie, v. 1, p. 295-334.

Gawlick, H.-J., Frisch, W., Vecsei, A., Steiger, T., and Böhm, F., 1999, The change from rifting to thrusting in the Northern Calcareous Alps as recorded in Jurassic sediments: Geologische Rundschau, v. 87, p. 644-657.

Gawlick, H.-J., Frisch, W., Missoni, S., and Suzuki, H., 2002, Middle to Late Jurassic radiolarite basins in the central part of the Northern Calcareous Alps as a key for the reconstruction of their early tectonic history-an overview: Memorie della Società Geologica Italiana, v. 57, p. 123-132.

Gawlick, H.-J., Schlanginweit, F., Ebli, O., and Suzuki, H., 2004, Die PlassenFormation (Kimmeridgium) des Krahstein (Steirisches Salzkammergut, Östereich) und ihre Unterkagerung: neue Daten zur Fazies, Biostratigraphie und Sedimentologie: Zentralblatt für Geologie und Paläontologie, Teil 1, v. 2003, p. 295-334.

Gawlick, H.-J., Schlagintweit, F., and Missoni, S., 2005, Die Barmsteinkalke der Typlokalität nordwestlich Hallein (hohes Tithonium bis tieferes Berriasium; Salzburger Kalkalpen)—Sedimentologie, Mikrofazies, Stratigraphie und Mikropaläontologie: neue Aspekte zur Interpretation der Entwicklungsgeschichte der Ober-Jura-Karbonatplattform und der tektonischen Interpretation der Hallstätter Zone von Hallein - Bad Dürrnberg: Neues Jahrbuch für Geologie und Paläontologie, Abhandlungen, v. 236, p. $351-421$.

Gawlik, H.-J., Suzuki, H., and Schlagintweit, F., 2006, Die ober-Trias- und JuraSedimentgesteine der Sarsteinalm und ihre Bedeutung für die tektonische Gliederung des Dachstein-Blockes (Salzkammergut, Nördliche Kalkalpen, Österreich): Neues Jahrbuch für Geologie und Paläontologie, Abhandlungen, v. 239, p. 101-160.

Gawlick, H.-J., Schlagintweit, F., and Missoni, S., 2007a, Das Ober-Jura Seichtwasser-Karbonat-Vorkommen der Drei Brüder am Wolfgangsee (Salzkammergut, Österreich): das westlichste Vorkommen der Wolfgangsee-Karbonatplattform südlich der Brunnwinkl-Schwelle am Nordrand des Tauglboden-Beckens: Journal of Alpine Geology, v. 48, p. $83-100$.

Gawlick, H.-J., Schlagintweit, F., and Suzuki, H., 2007b, Die Ober-Jura bis Unter-Kreide Schichtfolge des Gebietes Sandling-Höherstein (Salzkammergut, Österreich)-Implikationen zur Rekonstruktion des BlockPuzzles der zentralen Nördlichen Kalkalpen, der Gliederung der karbonatklastischen Radiolaritflyschbecken und der Entwicklung der Plassen-Karbonatplattform: Neues Jahrbuch für Geologie und Paläontologie, Abhandlungen, v. 243, p. 1-70.

Gawlick, H.-J., Frisch, W., Hoxha, L., Dumitrica, P., Krystyn, L., Lein, R., Missoni, S., and Schlagintweit, F., 2008, Mirdita Zone ophiolites and associated sediments in Albania reveal Neotethys Ocean origin: International Journal of Earth Sciences, v. 97, p. 865-881.

Gawlick, H.-J., Missoni, S., Schlagintweit, F., Suzuki, H., Frisch, W., Krystyn, L., Blau, J., and Lein, R., 2009, Jurassic Tectonostratigraphy of the Austroalpine Domain: Journal of Alpine Geology, v. 50, p. 1-152.

Gawlick, H.-J., Missoni, S., Schlagintweit, F., and Suzuki, H., 2012, Jurassic active continental margin deep-water basin and carbonate platform formation in the north-western Tethyan realm (Austria, Germany): Journal of Alpine Geology, v. 54, p. 189-292.

Golonka, J., 2002, Plate-tectonic Maps of the Phanerozoic, in Kiessling, W., Flügel, E., and Golonka, J., eds., Phanerozoic Reef Patterns: SEPM (Society for Sedimentary Geology) Special Publication, v. 72, p. 21-75.

Goričan, Š., 1987, Jurassic and Cretaceous radiolarians from the Budva zone (Montenegro, Yugoslavia): Revue de Micropaléontologie, v. 30, p. $177-196$

Goričan, Š., 1994, Jurassic and Cretaceous radiolarian biostratigraphy and sedimentary evolution of the Budva Zone (Dinarides, Montenegro): Mémoires de Géologie (Lausanne), v. 18, p. 1-120.

Goričan, Š., 1999, Theocapsomma medvednicensis Goričan, nov. sp., in Halamić, J., Goričan, Š., Slovenec, D., and Kolar-Jurkovsek, T., A Middle Jurassic Radiolarite-Clastic Succession from the Medvednica Mt. (NW Croatia): Geologia croatica, v. 52, p. 37.
Goričan, Š., Šmuc, A., and Baumgartner, P.O., 2003, Toarcian Radiolaria from Mt. Mangart (Slovenian-Italian border) and their paleoecological implications: Marine Micropaleontology, v. 49, p. 275-301.

Goričan, Š., Carter, E.S., Dumitrica, P., Whalen, P.A., Hori, R.S., De Wever, P., O'Dogherty, L., Matsuoka, A., and Guex, J., 2006, Catalogue and Systematics of Pliensbachian, Toarcian and Aalenian Radiolarian Genera and Species: Ljubljana, ZRC Publishing, Scientific Research Centre of the Slovenian Academy of Sciences and Arts, $446 \mathrm{p}$.

Goričan, Š., Pavšič, J., and Rožič, B., 2012, Bajocian to Tithonian age of radiolarian cherts in the Tolmin basin (NW Slovenia): Bulletin de la Societe Geologique de France, v. 183, p. 369-382.

Górka, H., 1989, Les Radiolaires du Campanien inférieur de Cracovie (Pologne): Acta Palaeontologica Polonica, v. 34, p. 327-354.

Grill, J., and Kozur, H., 1986, The first evidence of the Unuma echinatus radiolarian zone in the Rudabanya Mts. (northern Hungary): Geologisch Paläontologische Mitteilungen Innsbruck, v. 13, p. 239-275.

Haas, J, Kovacs, S., Krystyn, L., and Lein, R., 1995, Significance of Late Permian-Triassic facies zones in terrane reconstructions in the AlpineNorth Pannonian domain: Tectonophysics, v. 242, p. 19-40.

Haeckel, E., 1862, Die Radiolarien (Rhizopoda Radiaria). Eine Monographie: Berlin, Reimer, 572 p.

Haeckel, E., 1881, Entwurf eines Radiolarien-Systems auf Grund von Studien der Challenger-Radiolarien: Jenaische Zeitschrift für Naturwissenschaft, v. 15 , p. $418-472$

Halamić, J., Goričan, Š., Slovenec, D., and Kolar-Jurkovsek, T., 1999, A Middle Jurassic Radiolarite-Clastic Succession from the Medvednica Mt. (NW Croatia): Geologia Croatica, v. 52, p. 29-57.

Hatakeda, K., Suzuki, N., and Matsuoka, A., 2007, Quantitative morphological analyses and evolutionary history of the Middle Jurassic polycystine radiolarian genus Striatojaponocapsa Kozur: Marine Micropaleontology, v. 63 , p. $39-56$.

Hattori, I., 1987, Jurassic radiolarian fossils from the Nanjo Massif, Fukui Prefecture, central Japan: Bulletin of the Fukui Municipal Museum of Natural History, v. 34, p. 29-101.

Hattori, I., 1989, Jurassic radiolarians from manganese nodules at three sites in the western Nanjo Massif, Fukui Prefecture, central Japan: Memoirs of the Faculty of Education, Fukui University, Series 2, v. 2, p. 47-134.

Hattori, I., and Sakamoto, N., 1989, Geology and Jurassic Radiolarians from manganese nodules of the Kanmuriyama-Kanakusadake Area in the Nanjo Massif, Fukui Prefecture, central Japan: Bulletin of the Fukui Municipal Museum of Natural History, v. 36, p. 25-79.

Heitzer, I., 1930, Die Radiolarienfauna der mitteljurassischen Kieselmergel im Sonnwendgebirge: Jahrbuch der Geologischen Bundesanstalt, Wien, v. 80, p. 381-406.

Hori, N., 1999, Latest Jurassic radiolarians from the northeastern part of the Torinoko Block, Yamizo Mountains, central Japan: Science Reports of the Institute of Geoscience, University of Tsukuba, Section B: Geological Sciences, v. 20, p. 47-114.

Hull, D.M., 1997, Upper Jurassic Tethyan and southern boreal radiolarians from western North America: Micropaleontology, v. 43 (supplement 2), p. $1-202$.

Ichikawa, K., and Yao, A., 1976, Two new genera of Mesozoic cyrtoid radiolarians from Japan, in Takayanagi, Y., and Saito, T., eds., Progress in Micropaleontology, Special Publication: New-York, Micropaleontology Press, The American Museum of Natural History, p. 110-117.

Imoto, N., Tamaki, A., Tanabe, T., and Ishiga, H., 1982, An age determination on the basis of radiolarian biostratigraphy of a bedded manganese deposit at the Yumiyama Mine in the Tamba District, southwest Japan: News of Osaka Micropaleontologists, special volume, v. 5, p. 227-235.

Ishida, N., 2009, Jurassic to Early Cretaceous accretionary complexes and Upper Jurassic trench-slope basin deposits of the Southern Chichibu Terrane in the Itsuki-Gokanosho area, western Kyushu: News of Osaka Micropaleontologists, Special Volume, v. 14, p. 375-403.

Ishida, N., 2011, An exotic body of the Middle Jurassic Torinosu-type limestone in the Hikawa formation, southeastern Kanto Mountains, Japan: Memoir of the Fukui Prefectural Dinosaur Museum, v. 10, p. 103-112.

Jud, R., 1994, Biochronology and systematics of Early Cretaceous Radiolarian of the Western Tethys: Mémoires de Géologie (Lausanne), v. 19, p. 1-147.

Karamata, S., 2006, The geological development of the Balkan Peninsula related to the approach, collision and compression of Gondwanan and Eurasian units, in Robertson, A.H.F., and Mountrakis, D., eds., Tectonic Development of the Eastern Mediterranean Región: Geological Society of London, Special Publication, v. 260, p. 155-178.

Kawabata, K., 1988, New species of Latest Jurassic and Earliest Cretaceous radiolarians from the Sorachi Group in Hokkaido, Japan: Bulletin of the Osaka Museum of Natural History, v. 43, p. 1-13.

Kemkin, I.V., and Taketani, Y., 2004, New Radiolarian species from Late Jurassic chert-terrigenous deposits of the Taukha Terrane, Southern SikhoteAlin: Paleontological Research, v. 8, p. 325-336. 
Kido, S., Kawaguchi, I., Adachi, M., and Mizutani., S., 1982, On the Dictyomitrella (?) kamoensis-Pantanellium foveatum assemblage in the Mino area, central Japan: News of Osaka Micropaleontologists, Special Volume, v. 5, p. 195-210.

Kiessling, W., 1992, Radiolaria, in Kiessling, W., and Zeiss, A., New palaeontolgical data from the Hochstegen Marble (Tauern window, eastern Alps): Geologisch Paläontologische Mitteilungen Innsbruck, v. 18 , p. $189-192$.

Kiessling, W., 1999, Late Jurassic Radiolarians from the Antarctic Peninsula: Micropaleontology, Special Issues, v. 45, p. 1-96.

Kiessling, W., and Scasso, R., 1996, Ecological perspectives of Late Jurassic radiolarian faunas from Antarctic Peninsula, in Riccardi, A.C., ed., Advances in Jurassic Research 1-2, Zurich, Transtec, p. 317-326.

Kiessling, W., and Zeiss, A., 1992, New palaeontolgical data from the Hochstegen Marble (Tauern window, eastern Alps): Geologisch Paläontologische Mitteilungen Innsbruck, v. 18, p. 187-202.

Kishida, Y., and Hisada, K.-I., 1986, Radiolarian assemblages of the Sambosan Belt in the western part of the Kanto Mountains, central Japan: News of Osaka Micropaleontologists, Special Volume, v. 7, p. 25-34

Kishida, Y., and Sugano, K., 1982, Radiolarian zonation of Triassic and Jurassic in outer side of southwest Japan: News of Osaka Micropaleontologists, Special Volume, v. 5, p. 271-300.

Kito, N., and De Wever, P., 1992, Nouvelles espèces d'Hagiastridae (radiolaires) du Jurassique Moyen de Sicile (Italie): Revue de Micropaléontologie, v. 35, p. $127-141$.

Kocher, R., 1980, Stylocapsa oblongula Kocher, nov. sp., in Baumgartner, P. O. De Wever, P., and Kocher, R, Correlation of Tethyan Late Jurassic-Early Cretaceous radiolarian events: Cahiers de Micropaléontologie, v. 2, p. 62.

Kocher, R.N., 1981, Biochronostratigraphische Untersuchungen oberjurassischer radiolarien führender Gesteine, insbesondere der Südalpen: Mitteilungen aus dem geologischen Institut der Eidgenossischen Technischen Hochschule und der Universitat Zurich, Neue Folge, v. 234, p. 1-184.

Kojima, S., 1982, Some Jurassic, Triassic and Permian radiolarians from the eastern part of Takayama City, central Japan: News of Osaka Micropaleontologists, Special Volume, v. 5, p. 81-91.

Kokubo, S., and Matsuoka, A., 2009, Middle Jurassic radiolarians from siliceous mudstone of the Kamiyoshida Formation, the Northern Chichibu Belt in the Kanto Mountains, central Japan: News of Osaka Micropaleontologists, Special Volume, v. 14, p. 489-495.

Kozlova, G.E., 1966, Stichopilium bonum Kozlova, nov. sp., in Kozlova, G.E., and Gorbovetz, A.N., ,Radiolarians of the Upper Cretaceous and Upper Eocene deposits of the West Siberian Lowland: Proceedings of the All Union Petroleum Scientific Research Institute for Geological Survey (VNIGRI), v. 248, p. 113. [In Russian]

Kozur, H., 1984, New radiolarian taxa from the Triassic and Jurassic: Geologisch Paläontologische Mitteilungen Innsbruck, v. 13, p. 49-88.

Kozur, H., 1985, The radiolarian genus Eoxitus n. gen. from the Unuma echinatus Zone (Bajocian) of northern Hungary: Proceedings of the Koninklijke Nederlandse Akademie van Wetenschappen, Series B: Palaeontology, Geology, Physics, Chemistry, Anthropology, v. 88, p. 211-220.

Kozur, H., and Mostler, H., 1983, The polyphyletic origin and the classification of the Mesozoic saturnalids (Radiolaria): Geologisch Paläontologische Mitteilungen Innsbruck, v. 13, p. 1-47.

Kozur, H., and Mostler, H., 1986, Archaeodictyomitra annulata Kozur and Mostler, nov. sp., in Grill, J., and Kozur, H., The first evidence of the Unuma echinatus radiolarian zone in the Rudabanya Mts. (northern Hungary): Geologisch Paläontologische Mitteilungen Innsbruck, v. 13, p. 257.

Krainer, K., and Mostler, H., 1997, Die Lias-Beckenentwicklung der Unkener Synklinale (Nördliche Kalkalpen, Salzburg) unter besonderer Berücksichtigung der Scheibelberg Formation: Geologisch Paläontologische Mitteilungen Innsbruck, v. 22, p. 1-41.

Krainer, K., Mostler, H., and Haditsch, J.G., 1994, Jurassische Beckenbildung in den Nördlichen Kalkalpen bei Lofer (Salzburg) unter besonderer Berücksichtigung der Manganerz-Genese: Abhandlungen der Geologisches Bundesanstalt in Wien, v. 50, p. 257-293.

Krystyn, L., 1971, Stratigraphie, Fauna und Fazies der Klaus-Schichten (Aalenium-Oxford) in den östlichen Nordalpen: Verhandlungen der Geologischen Bundesanstalt in Wien, v. 3, 486-509.

Krystyn, L., 1972, Conodonten im Dachstein-Riffkalk (Nor) des Gosaukammes (Salzburg): Anzeiger der Österreichischen Akademie der Wissenschaften, Mathematisch-Naturwissenschaftliche Klasse, v. 109, p. 51-54.

Lein, R., 1985, Das Mesozoikum der Nördlichen Kalkalpen als Beispiel eines gerichteten Sedimentationsverlaufes infolge fortschreitender Krustenausdünnung: Archiv für Lagerstättenforschung der Geologischen Bundesanstalt, v. 6, p. 117-128.

Lein, R., 1987a, Zur Verbreitung der Hallstätter Zone beiderseits des PyhrnPasses: Oberösterreichische Geonachrichten, v. 2, p. 21-37.
Lein, R., 1987b, Evolution of the Northern Calcareous Alps during Triassic times, in Flügel, H.W., and Faupl, P., eds., Geodynamics of the Eastern Alps: Wien, Deuticke, p. 85-102.

Li, H.S., 1988, Early Jurassic (late Pliensbachian) Radiolaria from the Denggen area, Xizang (Tibet): Acta Micropalaeontologica Sinica, v. 5, p. 323-330.

Li, H.S., and Wu, H.R., 1985, Radiolaria from the Cretaceous Congdu Formation in southern Xizang (Tibet): Acta Micropalaeontologica Sinica, v. 2, p. 61-76.

Mandl, G.W., 1982, Jurassische Gleittektonik im Bereich der Hallstätter Zone zwischen Bad Ischl und Bad Aussee (Salzkammergut, Österreich): Mitteilungen der Gesellschaft der Geologie- und Bergbaustudenten in Österreich, v. 28, p. $55-76$.

Marcucci, M., and Prela, M., 1996, The Lumi Zi (Puke) section of the Kalur Cherts: radiolarian assemblages and comparison with other sections in northern Albania: Ofioliti, v. 21, p. 71-76.

Matsuoka, A., 1982, Jurassic two-segmented Nassellarians (Radiolaria) from Shikoku, Japan: Journal of Geosciences, Osaka City University, v. 25, p. 71-86.

Matsuoka, A., 1983, Middle and Late Jurassic radiolarian biostratigraphy in the Sakawa and adjacent areas, Shikoku, southwest Japan: Journal of Geosciences, Osaka City University, v. 26, p. 1-48.

Matsuoka, A., 1984, Late Jurassic four-segmented nassellarians (Radiolaria) from Shikoku, Japan: Journal of Geosciences, Osaka City University, v. 27, p. $143-153$.

Matsuoka, A., 1986a, Faunal change of radiolarians around the JurassicCretaceous boundary - with special reference to some multi-segmented nassellarians: Fossils (Tokyo), v. 40, p. 1-15.

Matsuoka, A., 1986b, Tricolocapsa yaoi Assemblage (Late Jurassic radiolarians) from the Togano Group in Shikoku, southwest Japan: Journal of Geosciences, Osaka City University, v. 29, p. 101-115.

Matsuoka, A., 1988, First appearance biohorizon of Tricolocapsa conexa within Jurassic siliceous mudstone sequences of the Kamiaso area in the Mino Terrane, central Japan; a correlation of radiolarian zones of the Middle Jurassic: The Journal of the Geological Society of Japan, v. 94, p. 583-590.

Matsuoka, A., 1991a, Middle Jurassic radiolarians from the western Pacific: Proceedings of the International Symposium on Shallow Tethys 3, Sendai, Japan, Saito Ho-on Kai Special Publication, p. 163-173.

Matsuoka, A., 1991b, Early Jurassic radiolarians from the Nanjo Massif in the Mino Terrane, central Japan. Part 1. Tricolocapsa, Stichocapsa and Minocapsa, n. gen.: Transactions and Proceedings of the Palaeontological Society of Japan, new Series, v. 161, p. 720-738.

Matsuoka, A., 1995, Middle Jurassic to Early Cretaceous radiolarian occurrences in Japan and the Western Pacific (ODP Sites 800-801), in Baumgartner, P.O., O'Dogherty, L., Goričan, Š., Urquhart, E., Pillevuit, A., and De Wever, P., eds., Mémoires de Géologie (Lausanne), v. 23, p. 937-966.

Matsuoka, A., 1998, Faunal composition of earliest Cretaceous (Berriasian) radiolaria from the Mariana Trench in the western Pacific: News of Osaka Micropaleontologists, Special Volume, v. 11, p. 165-187.

Matsuoka, A., 2004, Toarcian (Early Jurassic) radiolarian fauna from the Nanjo Massif in the Mino Terrane, central Japan: News of Osaka Micropaleontologists, Special Volume, v. 13, p. 69-87.

Matsuoka, A., and Baumgartner, P.O., 1997, Middle Jurassic radiolarians from the basal sediments at DSDP Site 534, Blake Bahama Basin, Northern Atlantic: News of Osaka Micropaleontologists, Special Volume, v. 10, p. 183-191.

Matsuoka, A., and Oji, T., 1990, Middle Jurassic radiolaria fossils from the Magisawa Formation in the Taro Belt, North Kitakami Mountains: The Journal of the Geological Society of Japan, v. 96, p. 239-241.

Matsuoka, A., and Yao, A., 1985, Latest Jurassic radiolarians from the Torinosu Group in southeast Japan: Journal of Geosciences, Osaka City University, v. 28 , p. $125-145$.

Missoni, S., 2003, Analyse der mittel- und oberjurassischen Becke- nentwicklung in den Berchtesgadener Kalkalpen-Stratigraphie, Fazies und Paläogeographie [Ph.D. thesis]: University of Leoben, Austria, $150 \mathrm{p}$.

Missoni, S., and Gawlick, H.-J., 2011a, Evidence for Jurassic subduction from the Northern Calcareous Alps (Berchtesgaden; Austroalpine, Germany): International Journal of Earth Sciences, v. 100, p. 1605-1631.

Missoni, S., and Gawlick, H.-J., 2011b, Jurassic mountain building and Mesozoic-Cenozoic geodynamic evolution of the Northern Calcareous Alps as proven in the Berchtesgaden Alps (Germany): Facies, v. 57, p. 137-186.

Missoni, S., Steiger, T., and Gawlick, H.-J., 2001, Das "Gschirrkopffenster" in den Berchtesgadener Kalkalpen (Deutchland) und seine Interpretation: Neurgebnisse auf der Basis von stratigraphischen und faziellen Untersuchungen: Mitteilungen der Gesellschaft der Geologie- und Bergbaustudenten in Österreich, v. 45, p. 89-110.

Mizutani, S., 1981, A Jurassic formation in the Hida-Kanayama Area, central Japan: Bulletin of the Mizunami Fossil Museum, v. 8, p. 147-190.

Mizutani, S., and Kido, S., 1983, Radiolarians in Middle Jurassic siliceous shale from Kamiaso, Gifu Prefecture, central Japan: Transactions and Proceedings of the Palaeontological Society of Japan, New Series, v. 132, p. 253-262. 
Mizutani, S., and Koike, T., 1982, Radiolarians in the Jurassic siliceous shale and the Triassic bedded chert of Unuma, Kagamigahara City, Gifu Prefecture, central Japan: News of Osaka Micropaleontologists, Special Volume, v. 5, p. 117-134

Müller, J., 1858, Über die Thalassicollen, Polycystinen und Acanthometren des Mittelmeeres: Abhandlungen der Königlich Preussischen Akademie der Wissenschaften zu Berlin, p. 1-62.

Nakae, S., and Komuro, K., 2005, Radiolarian age of chert-hosted bedded manganese deposits from the Gen-otani mine in the Tamba district, northern Kyoto City, Japan: Resource Geology, v. 55, p. 311-320.

Nakaseko, K., and Nishimura, A., 1979, Amphipyndax awaensis Nakaseko and Nishimura, nov. sp., in Nakaseko, K., Nishimura, A., and Sugano, K., Cretaceous Radiolaria in the Shimanto Belt, Japan: News of Osaka Micropaleontologists, Special Volume, v. 2, p. 21.

Nakaseko, K., and Nishimura, A., 1981, Upper Jurassic and Cretaceous Radiolaria from the Shimanto Group in Southwest Japan: Science Reports, College of General Education Osaka University, v. 30, p. 133-203.

Nakaseko, K., Nishimura, A., and Sugano, K., 1979, Cretaceous Radiolaria in the Shimanto Belt, Japan: News of Osaka Micropaleontologists, Special Volume, v. 2, p. 1-49.

Nishihara, C., 2009, Change of Middle Jurassic (Bajocian) radiolarian assemblages: Case study in the Unuma section, Mino Terrane, central Japan: News of Osaka Micropaleontologists, Special Volume, v. 14, p. 433-470.

Nishihara, C., and Yao, A., 2005a, Faunal change of Middle Jurassic (Bajocian) radiolarians from manganese carbonate nodules in the Inuyama area, central Japan: Journal of Geosciences, Osaka City University, v. 48, p. 109-121. [In Japanese]

Nishihara, C., and Yao, A., 2005b, Faunal change of Middle Jurassic (Bajocian) radiolarians in the Inuyama area of the Mino Terrane: Fossils (Tokyo), v. 78, v. 32-39. [In Japanese]

Nishizono, Y., 2001, Jurassic radiolarians from the Sakamoto Formation in the Kurosegawa Terrane, Kyushu, southwest Japan: News of Osaka Micropaleontologists, Special Volume, v. 12, p. 203-214.

Nishizono, Y., Ohishi, A., Sato, T., and Murata, M., 1982, Radiolarian fauna from the Paleozoic and Mesozoic formations, distributed along the mid-stream of Kuma River, Kyushu, Japan: News of Osaka Micropaleontologists, Special Volume, v. 5, p. 311-326.

O'Dogherty, L., 1994, Biochronology and Paleontology of Mid-Cretaceous Radiolarians from Northern Apennines (Italy) and Betic Cordillera (Spain): Mémoires de Géologie (Lausanne), v. 21, p. 1-415.

O'Dogherty, L., and Gawlick, H.J., 2008, Pliensbachian Radiolaria in the Teltschengraben (Northern Calcareous Alps, Salzkammergut area, Austria) and their evidence for the reconstruction of Liassic Tethys: Stratigraphy, v. 5, p. 63-81.

O'Dogherty, L., Goričan, Š., and Dumitrica, P., 2006, Radiolaria, in O’Dogherty, L., Bill, M., Goričan, Š., Dumitrica, P., and Masson, H., Bathonian radiolarians from an ophiolitic melange of the Alpine Tethys (Gets Nappe, Swiss-French Alps): Micropaleontology, v. 51, p. 425-485.

O’Dogherty, L., Bill, M., Goričan, Š., Dumitrica, P., and Masson, H., 2006, Bathonian radiolarians from an ophiolitic melange of the Alpine Tethys (Gets Nappe, Swiss-French Alps): Micropaleontology, v. 51, p. $425-485$.

O’Dogherty, L., Carter, E.S., Dumitrica, P., Goričan, Š., De Wever, P., Bandini, A.N., Baumgartner, P.O., and Matsuoka, A., 2009, Catalogue of Mesozoic radiolarian genera; Part 2, Jurassic-Cretaceous: Geodiversitas, v. 31, p. 271-356.

O’Dogherty, L., De Wever, P., Goričan, Š., Carter, E.S., and Dumitrica, P., 2011, Stratigraphic ranges of Mesozoic radiolarian families: Palaeoworld, v. 20 , p. $102-115$

Okada, H., Tarduno, J.A., Nakaseko, K., Nishimura, A., Sliter, W.V., and Okada, H., 1989, Microfossil assemblage from the Late Jurassic to Early Cretaceous Nikoro Pelagic Sediments, Tokoro Belt, Hokkaido, Japan: Memoirs of the Faculty of Science, Kyushu University, Series D, Earth and Planetary Sciences, v. 27, p. 193-214.

Özdikmen, H., 2009, Substitute names for some unicellular animal taxa (Protozoa): Munis Entomology and Zoology, v. 4, p. 233-256.

Ožvoldová, L., 1975, Upper Jurassic radiolarians from the Kisuca Series in the Klippen Belt: Západné Karpaty, Séria Paleontológia, v. 1, p. 73-86.

Ožvoldová, L., 1992, The discovery of a Callovian radiolarian association in the Upper Posidonia Beds of the Pieniny succession of the Klippen Belt (Western Carpathians): Geologica Carpathica, v. 43, p. 111-122.

Ožvoldová, L., 1997, Genus Fultacapsa Ožvoldová, nov. gen, in Ožvoldová, L., and Frantová, L., Jurassic radiolarites from the eastern part of the Pieniny Klippen Belt (Western Carpathians): Geologica Carpathica, v. 48, p. 59.

Ožvoldová, L., and Frantová, L., 1997, Jurassic radiolarites from the eastern part of the Pieniny Klippen Belt (Western Carpathians): Geologica Carpathica, v. 48 , p. 49-61.
Parona, C.F., 1890, Radiolarie nei noduli selciosi del calcare giurese di Cittiglio presso Laverno: Bollettino Della Societa Geologica Italiana, v. 9, p. 132-175.

Pessagno, E.A., 1971, Jurassic and Cretaceous Hagiastridae from the Blake-Bahama Basin (Site 5A, JOIDES Leg 1) and the Great Valley Sequence, California Coast Ranges: Bulletins of American Paleontology, v. 264 , p. $5-83$

Pessagno, E.A., 1972, Cretaceous Radiolaria. Part I: The Phaseliformidae, new family and other Spongodiscacea from the Upper Cretaceous portion of the Great Valley Sequence, part II; Pseudoaulophacidae Riedel from the Cretaceous of California and the Blake-Bahama Basin (JOIDES leg 1): Bulletins of American Paleontology, v. 270, p. 269-328.

Pessagno, E.A., 1973, Upper Cretaceous Spumellariina from the Great Valley Sequence, California Coast Ranges: Bulletins of American Paleontology, v. 276 , p. $49-102$.

Pessagno, E.A., 1976, Radiolarian zonation and stratigraphy of the Upper Cretaceous portion of the Great Valley Sequence, California Coast Ranges: Micropaleontology, Special Publication, v. 2, p. 1-95.

Pessagno, E.A., 1977a, Upper Jurassic Radiolaria and radiolarian biostratigraphy of the California Coast Ranges: Micropaleontology, v. 23, p. 56-113.

Pessagno, E.A., 1977b, Lower Cretaceous radiolarian biostratigraphy of the Great Valley Sequence and Franciscan Complex, California Coast Ranges: Cushman Foundation for Foraminiferal Research, Special Publication, v. 15 , p. $1-87$.

Pessagno, E.A., 1979, Radiolaria, in Pessagno, E.A., Finch, W., and Abbott, P.L., Upper Triassic Radiolaria from the San Hipolito Formation, Baja California: Micropaleontology, v. 25, p. 160-197.

Pessagno, E.A., and Blome, C., 1980, Upper Triassic and Jurassic Pantanelliinae from California, Oregon and British Columbia: Micropaleontology, v. 26, p. 225-273.

Pessagno, E.A.J., and Hull, D.M., 2002, Upper Jurassic (Oxfordian) Radiolaria from the Sula Islands (East Indies): Their taxonomic, biostratigraphic, chronostratigraphic and paleobiogeographic significance: Micropaleontology, v. 48, p. 229-256.

Pessagno, E.A., and Whalen, P., 1982, Lower and Middle Jurassic Radiolaria (multicyrtid Nassellariina) from California, east-central Oregon and the Queen Charlotte Islands, B. C.: Micropaleontology, v. 28, p. 111-169.

Pessagno, E.A., Finch, W., and Abbott, P.L., 1979, Upper Triassic Radiolaria from the San Hipolito Formation, Baja California: Micropaleontology, v. 25 , p. $160-197$.

Pessagno, E.A., Blome, C., and Longoria, J., 1984, A revised radiolarian zonation from Upper Jurassic of western North America: Bulletins of American Paleontology, v. 320, p. 1-51.

Pessagno, E.A., Blome, C.D., and Hull, D.M., 1993, Radiolaria, in Pessagno, E. A., Blome, C. D., Hull, D. M., and Six, W. M., Jurassic Radiolaria from the Josephine ophiolite and overlying strata, Smith River subterrane (Klamath Mountains), northwestern California and southwestern Oregon: Micropaleontology, v. 39, p. 93-166.

Pessagno, E.A., Blome, C.D., Hull, D.M., and Six, W.M., 1993, Jurassic Radiolaria from the Josephine ophiolite and overlying strata, Smith River subterrane (Klamath Mountains), northwestern California and southwestern Oregon: Micropaleontology, v. 39, p. 93-166.

Plöchinger, B., 1974, Gravitativ transportiertes permisches Haselgebirge in den Oberalmer Schichten (Tithonium, Salzburg): Verhandlungen der Geologischen Bundesanstalt in Wien, v. 1974, 71-88.

Plöchinger, B., 1976, Die Oberalmer Schichten und die Platznahme der Hallstätter Masse in der Zone Hallein-Berchtesgaden: Neues Jahrbuch für Geologie und Paläontologie, Abhandlungen, v. 151, p. 304-324.

Riedel, W.R., 1967, Some new families of Radiolaria: Proceedings of the Geological Society of London, v. 1640, p. 148-149.

Riedel, W.R., 1971, Systematic classification of polycystine Radiolaria, in Funnell, B.M., and Riedel, W.R., eds., The Micropalaeontology of Oceans, Cambridge, UK, Cambridge University Press, p. 649-660.

Riedel, W.R., and Sanfilippo, A., 1974, Radiolaria from the southern Indian Ocean, DSDP Leg 26, in Davies, T.A., Luyendyk, B.P., et al., eds., Initial Reports of the Deep Sea Drilling Project, Volume 26: Washington, D.C., U.S. Government Printing Office, p. 771-814.

Robertson, A.H., 2012, Late Palaeozoic-Cenozoic tectonic development of Greece and Albania in the context of alternative reconstructions of Tethys in the Eastern Mediterranean region: International Geology Review, v. 54, p. $373-454$.

Robin, C., Goričan, Š., Guillocheau, F., Razin, P., Dromart, G., and Mosaffa, H., 2010, Mesozoic deep-water carbonate deposits from the southern Tethyan passive margin in Iran (Pichakun nappes, Neyriz area): biostratigraphy, facies sedimentology and sequence stratigraphy, in Leturmy, P., and Robin, C., eds., Tectonic and Stratigraphic Evolution of Zagros and Makran during 
the Mesozoic-Cenozoic: Special Publications of the Geological Society of London, v. 330, p. 179-210.

Rüst, D., 1885, Beiträge zur Kenntniss der fossilen Radiolarien aus Gesteinen des Jura: Palaeontographica, v. 31, p. 269-321.

Rüst, D., 1898, Neue Beiträge zur Kenntniss der Fossilen Radiolarien aus Gesteinen des Jura und der Kreide: Palaeontographica, v. 45, p. 1-67.

Sashida, K., Igo, H., Igo, H., Takizawa, S., Hisada, K.-I., Shibata, T., Tsukada, K., and Nishimura, H., 1982, On the Jurassic radiolarian assemblages in the Kanto district: News of Osaka Micropaleontologists, Special Volume, v. 5, p. 51-66.

Schaaf, A., 1981, Late Early Cretaceous Radiolaria from Deep Sea Drilling Project Leg 62, in Thiede, J., Vallier, T.L., et al., eds., Initial Reports of the Deep Sea Drilling Project, Volume 62: Washington, D.C., U.S. Government Printing Office, p. 419-470.

Schlager, W., and Schlager, M., 1973, Clastic sediments associated with radiolarites (Tauglbodenschichten, Upper Jurassic, Eastern Alps): Sedimentology, v. 20, p. 65-89.

Schmid, S.M., Bernoulli, D., Fügenschuh, B., Matenco, L., Schefer, S., Schuster, R., Tischler, M., and Ustaszewski, K., 2008, The AlpineCarpathian-Dinaride-orogenic system: correlation and evolution of tectonic units: Swiss Journal of Geosciences, v. 101, p. 139-183.

Šegvić, B., Kukoć, D., Dragičević, I., Vranjković, A., Brčić, V., Goričan, Š., Babajić, E., and Hrvatović, H., 2014, New record of Middle Jurassic radiolarians and evidence of Neotethyan dynamics documented in a mélange from the Central Dinaridic Ophiolite belt (CDOB, NE Bosnia and Herzegovina): Ofioliti, v. 39, p. 31-41.

Sher, S.A., 1973, The classification of Tetylenchus Filipjev, 1939, Leipotylenchus $\mathrm{n}$. gen. (Leipotylenchinae $\mathrm{n}$. subf.) and Triversus n. gen. (Nematoda: Tylenchoidae): Nematologica, v. 19, p. 318-325.

Šmuc, A., and Goričan, Š., 2005, The Jurassic sedimentary evolution of a carbonate platform into a deep-water basin, Mt. Mangart (Slovenian-Italian border): Rivista Italiana di Paleontologia e Stratigrafia, v. 111, p. 45-70.

Squinabol, S., 1903, Le Radiolarie dei noduli selciosi nella Scaglia degli Euganei. Contribuzione I.: Rivista Italiana di Paleontologia, v. 9, p. 105-151.

Squinabol, S., 1904, Radiolarie Cretacee degli Euganei: Atti e Memorie della reale Accademia di Scienze, Lettere ed Arti in Padova, Nuova Serie, v. 20, p. $171-244$.

Squinabol, S., 1914, Contributo alla conoscenza dei Radiolarii fossili del Veneto. Appendice - Di un genera di Radiolari caratteristico del Secondario: Memorie dell'Istituto Geologico della reale Universita di Padova, v. 2, p. 249-306.

Steiger, T., 1992, Systematik, stratigraphie und Palökologie der Radiolarien des Oberjura-Unterkreiden-Grenzbereiches im Osterhorn-Tirolikum (Nördliche Kalkalpen, Salzburg und Bayern: Zitteliana, v. 19, p. 3-188.

Suzuki, H., 1997, Drei neue Radiolarienarten in der Canoptum-Vergesellschaftung aus dem Unterjura des Kanoashi-Komplexes Südwestjapans: Neues Jahrbuch für Geologie und Paläontologie, Monatshefte, v. 6, p. 361-369.

Suzuki, H., and Gawlick, H.-J., 2003, Biostratigraphie und Taxonomie der Radiolarien aus den Kieselsedimenten der Blaa Alm und nödlich des Loser (Nördliche Kalkalpen, Callovieum-Oxfordium): Mitteilungen der Gesellschaft der Geologie- und Bergbaustudenten in Österreich, v. 46, p. 137-228.

Suzuki, H., and Gawlick, H.-J., 2009, Jurassic radiolarians from cherty limestones below the Hallstatt salt mine (Northern Calcareous Alps, Austria): Neues Jahrbuch für Geologie und Paläontologie, Abhandlungen, v. 251, p. 155-197.

Takahashi, O., and Ishii, A., 1995, Radiolarian Assemblage-zones in the Jurassic and Cretaceous Sequence in the Kanto Mountains, central Japan: Memoirs of the Faculty of Science, Kyushu University, Series D, Earth and Planetary Sciences, v. 24, p. $49-85$.

Takemura, A., 1986, Classification of Jurassic Nassellarians (Radiolaria): Palaeontographica, Abteilung A: Palaozoologie-Stratigraphie, v. 195, p. 29-74.

Tan, S.H., 1927, Over de samenstelling en het onstaan van krijt- en mergelgesteenten van de Molukken: Jaarboek van het mijnwezen in Nederlandsch Oost-Indie, Jaargang 55 (1926), Verhandelingen, 3rd gedeelte, p. 5-165.

Tollmann, A., 1981, Oberjurassische Gleittektonik als Hauptformungsprozess der Hallstätter Region und neue Daten zur Gesamttektonik der Nördlichen Kalkalpen in den Ostalpen: Mitteilungen der Österreichischen Geologischen Gesellschaft, v. 74(75), p. 167-195.

Tollmann, A., 1985, Geologie von Österreich, Band 2: Wien, Deuticke, 718 p.

Tollmann, A., 1987, Late Jurassic/Neocomian gravitational tectonics in the Northern Calcareous Alps in Austria, in Flügel, H.W., and Faupl, P., eds., Geodynamics of the Eastern Alps: Wien, Deuticke, p. 112-125.

Tumanda, F., 1989, Cretaceous radiolarian biostratigraphy in the Esashi Mountain area, Northern Hokkaido, Japan: Science Reports of the Institute of Geoscience, University of Tsukuba, Section B: Geological Sciences, v. 10, p. 1-44.

Vinassa de Regny, P.E., 1901, Radiolari Cretacei dell'Isola di Karpathos: Memorie della reale Accademia delle Scienze dell'Istituto di Bologna, v. 9, p. 497-512.

Vishnevskaya, V.S., 1991, The discovery of Jurassic Radiolaria Amphipyndacidae in the far east of the USSR, in Tochilina, S.V., ed., Paleontological and Stratigraphic Investigation of Phanerozoic in the Far Eastern Region (by the results of radiolarian analysis for mapping). Collected proceedings, Vladivostok, Far East Division of the Academy of Science of the USSR, Pacific Oceanological Institute, p. 63-67. [In Russian]

Vishnevskaya, V.S., 1991, Genus Schaafella Vishnevskaya, nov. sp., in Basov, V.A., and Vishnevskaya, V.S. Upper Mesozoic Stratigraphy of the Pacific Ocean, Circum-Pacific Council for Energy and Mineral Resources, Moscow, Nauka, p. 166. [In Russian].

Vishnevskaya, V.S., 2001, Jurassic to Cretaceous Radiolarian Biostratigraphy of Russia, Moscow, GEOS, 376 p.

Vishnevskaya, V.S., and Murchey, B.L., 2002, Climatic affinity and possible correlation of some Jurassic to Lower Cretaceous radiolarian assemblages from Russia and North America: Micropaleontology, v. 48, p. 89-111.

Vlahović, I., Tišljar, J., Velić, I., and Matičec, D., 2005, Evolution of the Adriatic Carbonate Platform: Palaeogeography, main events and depositional dynamics: Palaeogeography, Palaeoclimatology, Palaeoecology, v. 220, p. $330-360$

Wakita, K., 1982, Jurassic radiolarians from Kuzuryo-ko- Gujo-hachiman area: News of Osaka Micropaleontologists, Special Volume, v. 5, p. 153-171.

Wakita, K., 1988, Early Cretaceous melange in the Hida-Kanayama area, central Japan: Bulletin of the Geological Survey of Japan, v. 39, p. 367-421.

Whalen, P.A., and Carter, E.S., 1998, Genus Solidea Whalen and Carter, nov. gen., in Carter, E.S., Whalen, P.A., and Guex, J., Biochronology and paleontology of Lower Jurassic (Hettangian and Sinemurian) radiolarians, Queen Charlotte Islands, British Columbia: Geological Survey of Canada, Bulletin, v. 496, p. 81.

Whalen, P.A., and Carter, E.S., 2002, Pliensbachian (Lower Jurassic) Radiolaria from Baja California Sur, Mexico: Micropaleontology, v. 48, p. 97-151.

Widz, D., and De Wever, P., 1993, Nouveaux Nassellaires (Radiolaria) des radiolarites jurassiques de la coupe de Szeligowy Potok (Zones de Klippes de Pieniny, Carpathes Occidentales, Pologne): Revue de Micropaléontologie, v. 36, p. 77-91.

Wu, H.R., 1993, Upper Jurassic and Lower Cretaceous radiolarians of Xialu chert, Yarlung Zangbo ophiolite belt, southern Tibet, in Blueford, J.R., and Murchey, B.L., eds., Radiolaria of Giant and Subgiant Fields in Asia. Nazarov Memorial Volume: Micropaleontology, Special Publication, v. 6, p. 115-136.

Wu, H.R., 2000, New data of the Late Jurassic nassellarian (Radiolaria) from the Xialu chert in southern Tibet: Geoscience (Beijing), v. 14, p. 301-306.

Wu, H.R., and Li., H.S., 1982, The Radiolaria of the olistostrome of Zongzhuo Formation, Gyangze, South Xizang, Tibet: Acta Palaeontologica Sinica, v. 21 , p. $64-71$.

Yamamoto, H., Mizutani, S., and Kagami, H., 1985, Middle Jurassic radiolarians from Blake Bahama Basin, West Atlantic Ocean: Bulletin of the Nagoya University, Furukawa Museum, v. 1, p. 25-49.

Yang, Q., 1993, Taxonomic Studies of Upper Jurassic (Tithonian) Radiolaria from the Taman Formation, east-central Mexico: Palaeoworld, v. 3, p. 1-164.

Yang, Q., and Wang, Y.J., 1990, A taxonomic study of Upper Jurassic radiolarian from Rutog county, Xizang (Tibet): Acta Micropalaeontologica Sinica, v. 7, p. 195-218.

Yao, A., 1972, Radiolarian fauna from the Mino Belt in the northern part of the Inuyama Area, central Japan, Part I: Spongosaturnalids: Journal of Geosciences, Osaka City University, v. 15, p. 21-65.

Yao, A., 1979, Radiolarian fauna from the Mino Belt in the northern part of the Inuyama Area, central Japan, Part II: Nassellaria 1: Journal of Geosciences, Osaka City University, v. 22, p. 21-72.

Yao, A., 1982, Middle Triassic to Early Jurassic radiolarians from the Inuyama area, central Japan: Journal of Geosciences, Osaka City University, v. 25, p. 53-70.

Yao, A., 1997, Faunal change of Early-Middle Jurassic radiolarians: News of Osaka Micropaleontologists, Special Volume, v. 10, p. 155-182.

Yeh, K.Y., 1987a, Taxonomic studies of Lower Jurassic Radiolaria from east-central Oregon: Special Publication of the National Museum of Natural Science, Taiwan, v. 2, p. 1-169.

Yeh, K.Y., 1987b, A revised classification for the family Canoptidae (Radiolaria): Memoirs of the Geological Society of China, v. 8, p. 63-72.

Yeh, K.Y., 2009, A middle Jurassic Radiolarian fauna from South Fork Member of Snowshoe Formation, east-central Oregon: Collection and Research, National Museum of Natural Science, Taiwan, v. 22, p. 15-125.

Yeh, K.Y., 2011, A Middle Jurassic (upper Bajocian) Radiolarian Assemblage from Snowshoe Formation, east-central Oregon: Collection and Research, National Museum of Natural Science, Taiwan, v. 24, p. 1-77.

Ziabrev, S.V., Aitchison, J.C., Abrajevitch, A.V., Badengzhu, A., Davis, A.M., and Luo, H., 2004, Bainang Terrane, Yarlung-Tsangpo suture, southern Tibet (Xizang, China): a record of intra-Neotethyan subduction-accretion processes preserved on the roof of the world: Journal of the Geological Society of London, v. 161, p. 523-539.

Accepted 27 December 2014 